\title{
Direct C-H Alkenylation of Functionalized Pyrazoles
}

\section{Supporting Information}

Su Jin Han, Hyun Tae Kim, Jung Min Joo*

Department of Chemistry and Chemistry Institute of Functional Materials, Pusan National University, Busan 46421, Republic of Korea

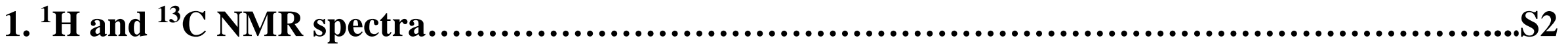

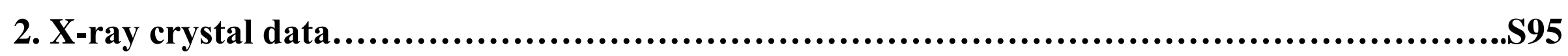



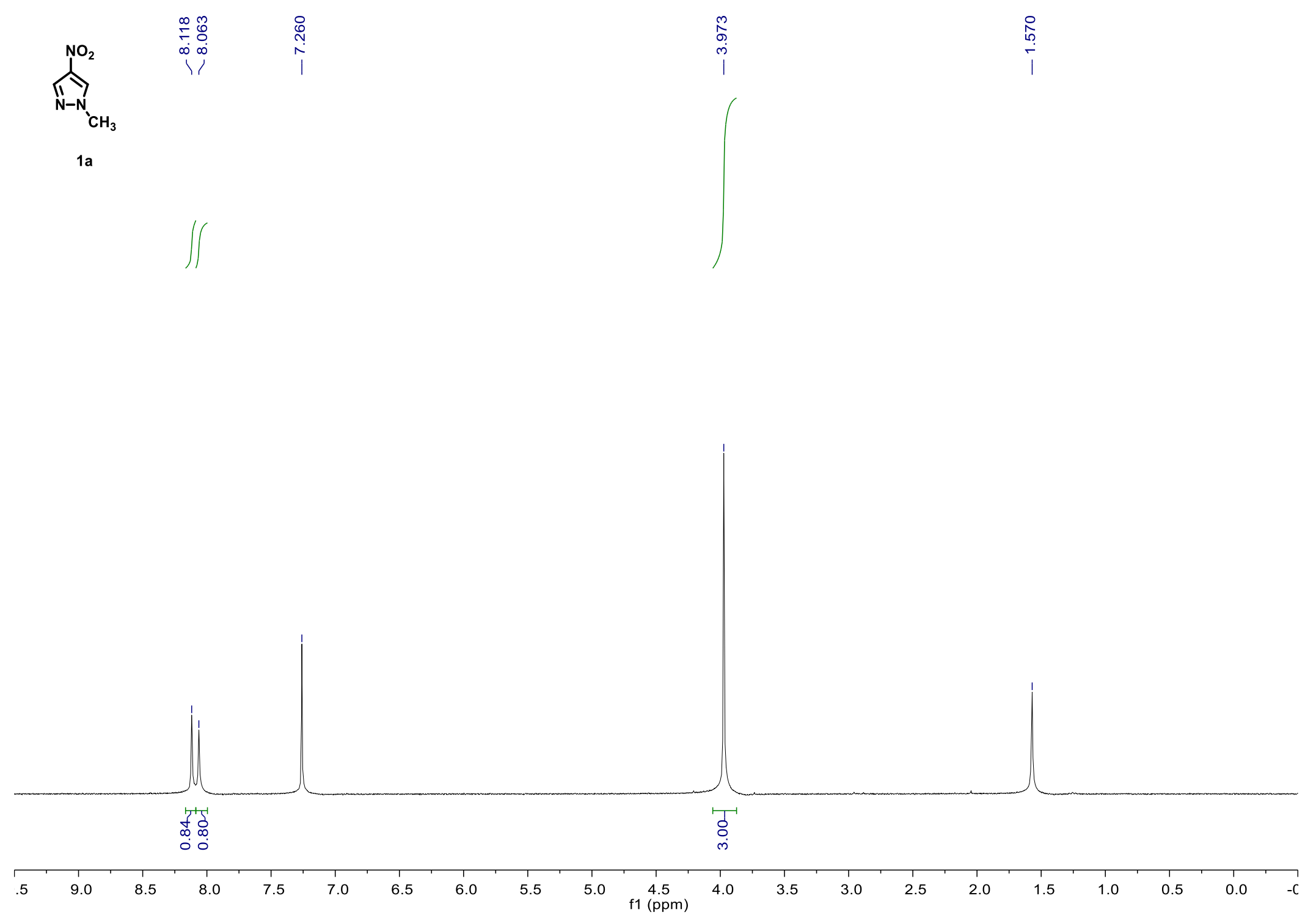


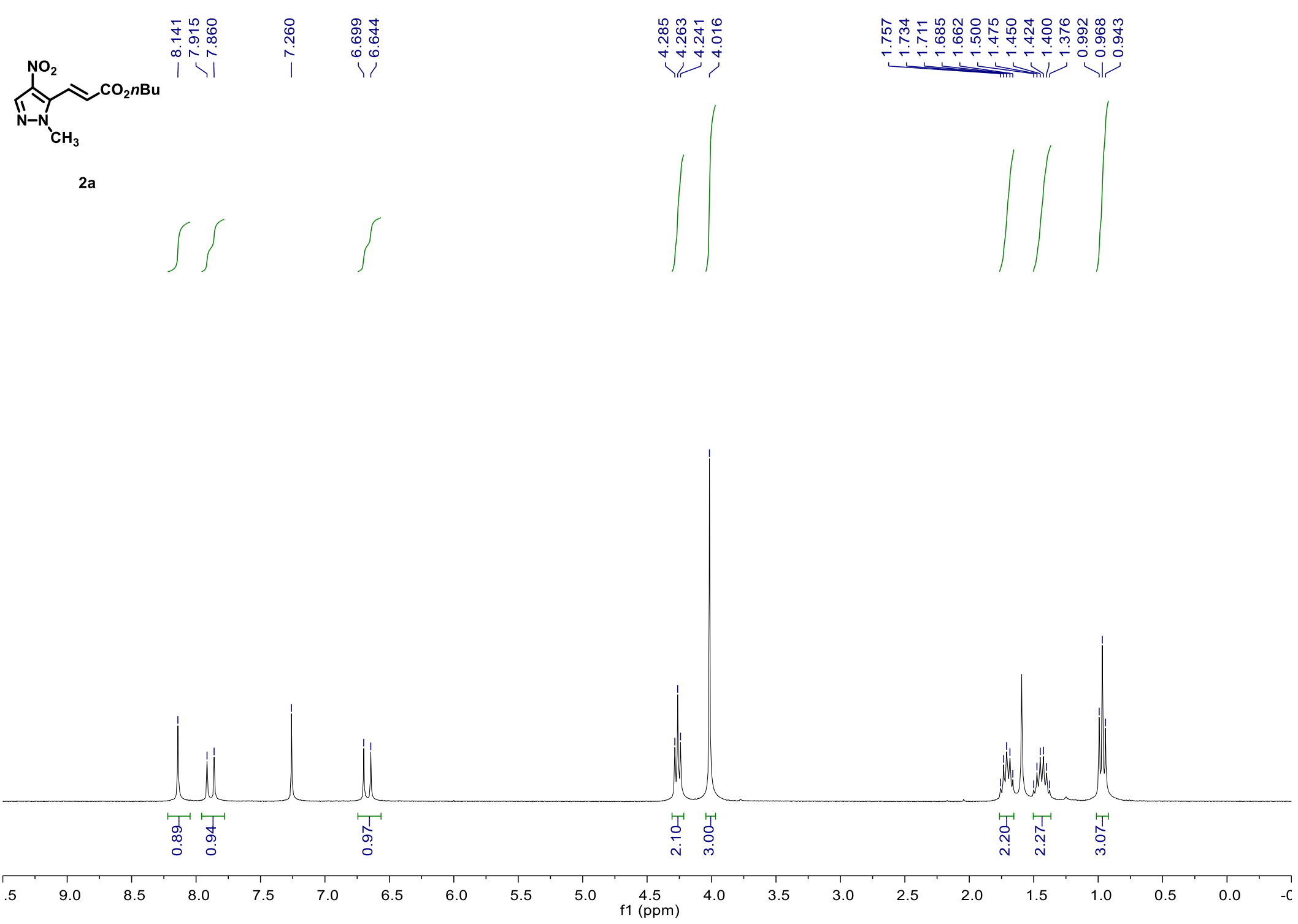




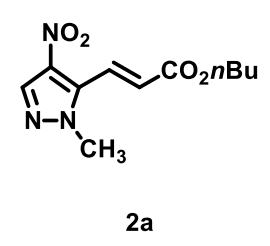

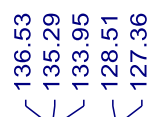

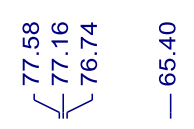

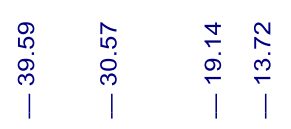
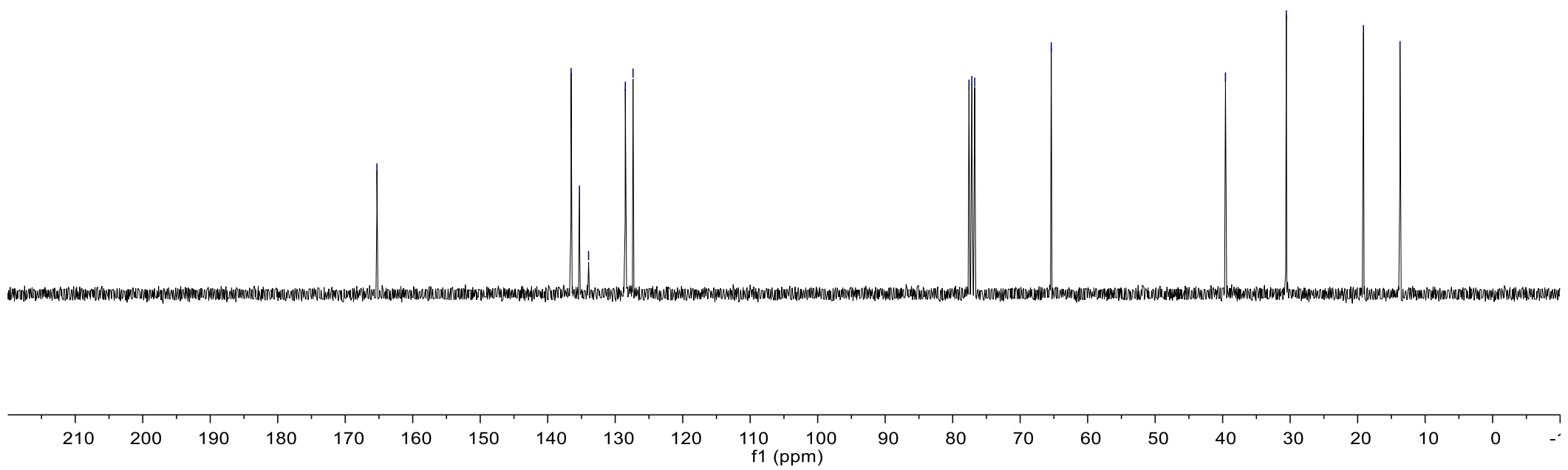

S4 


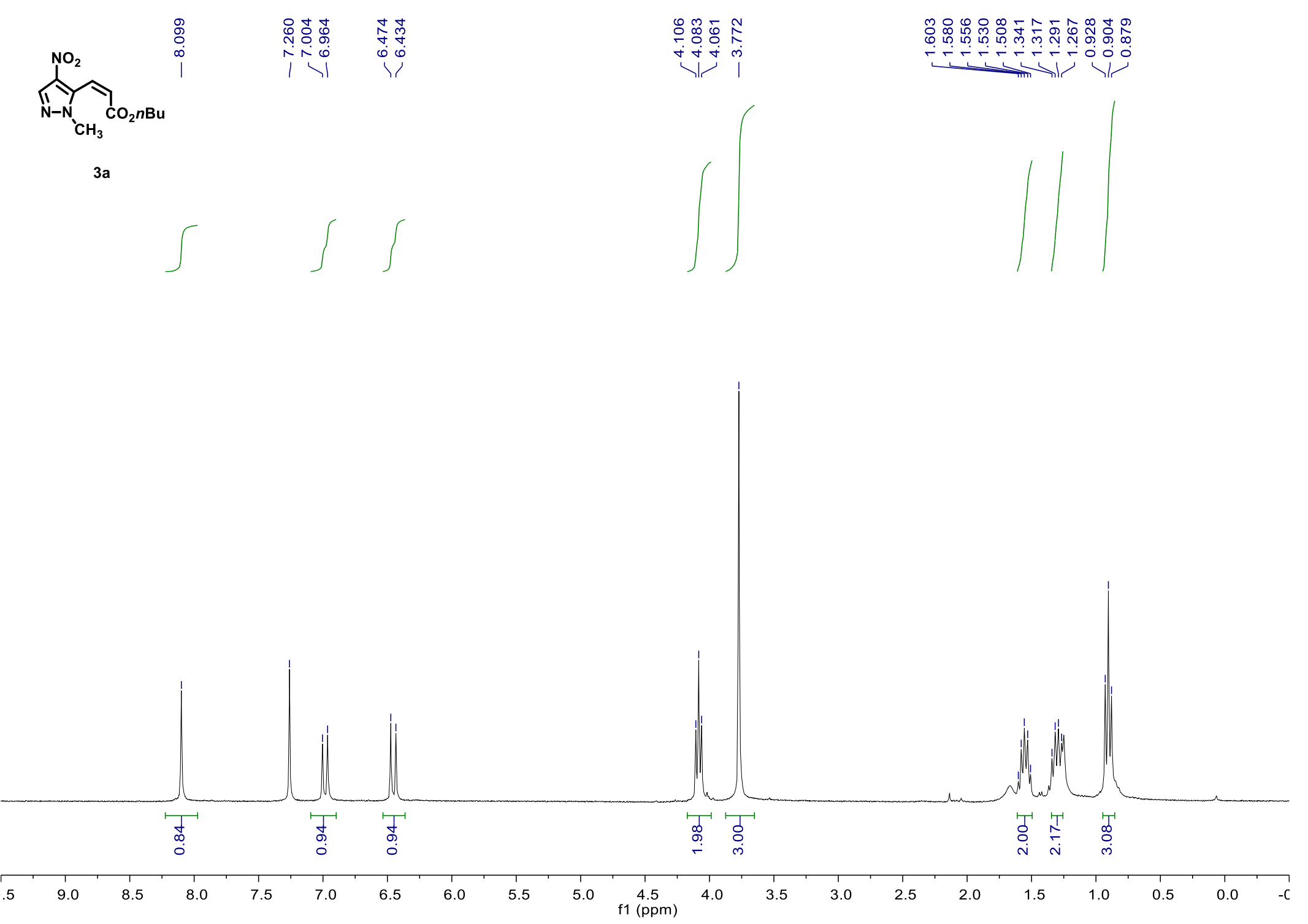




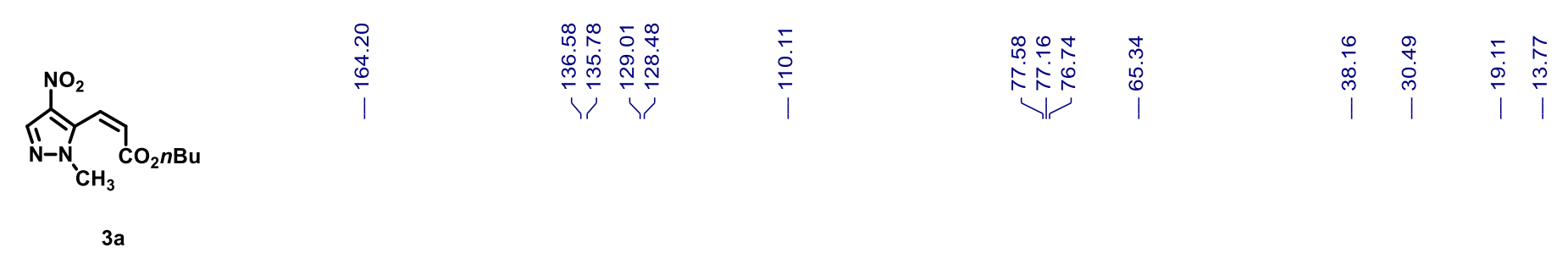

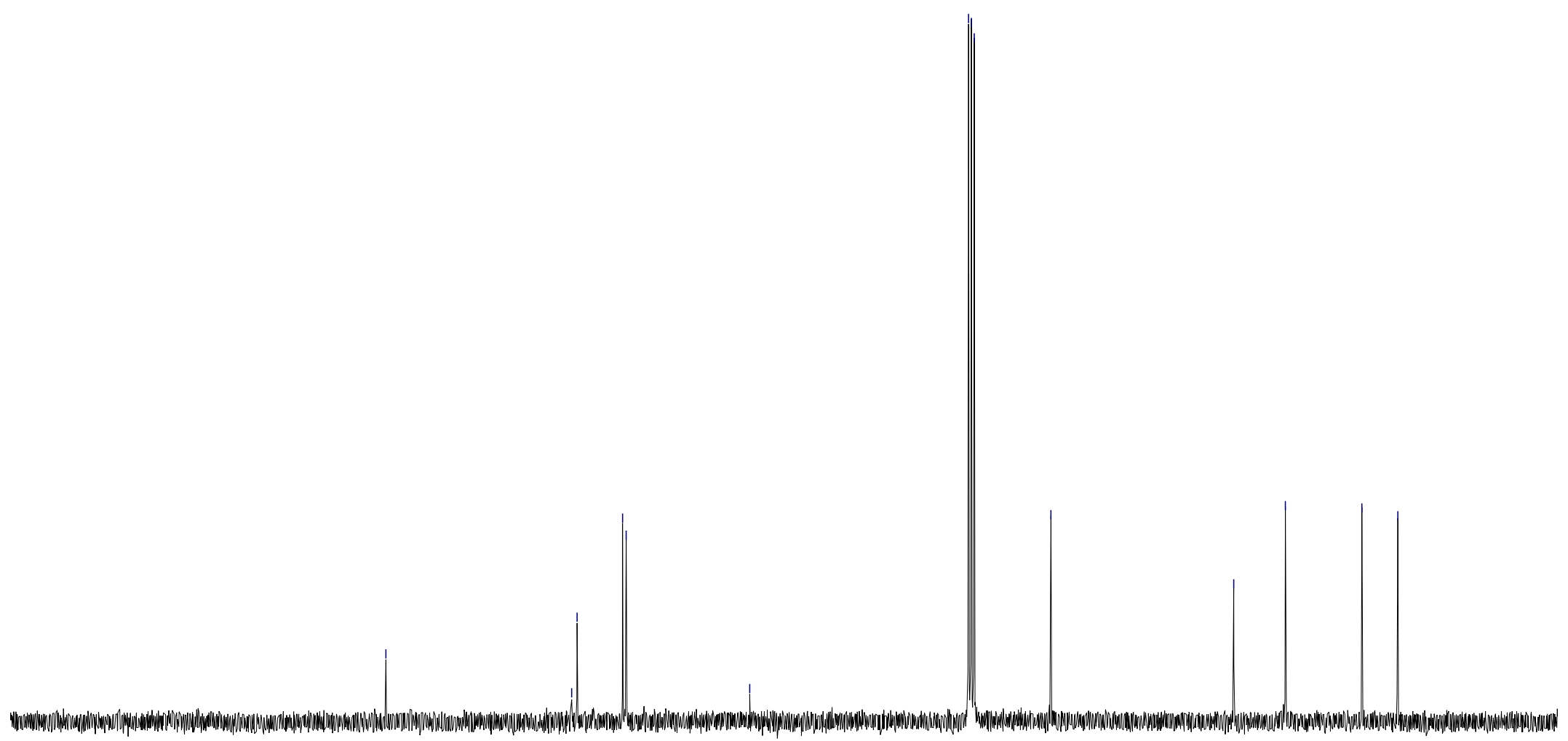




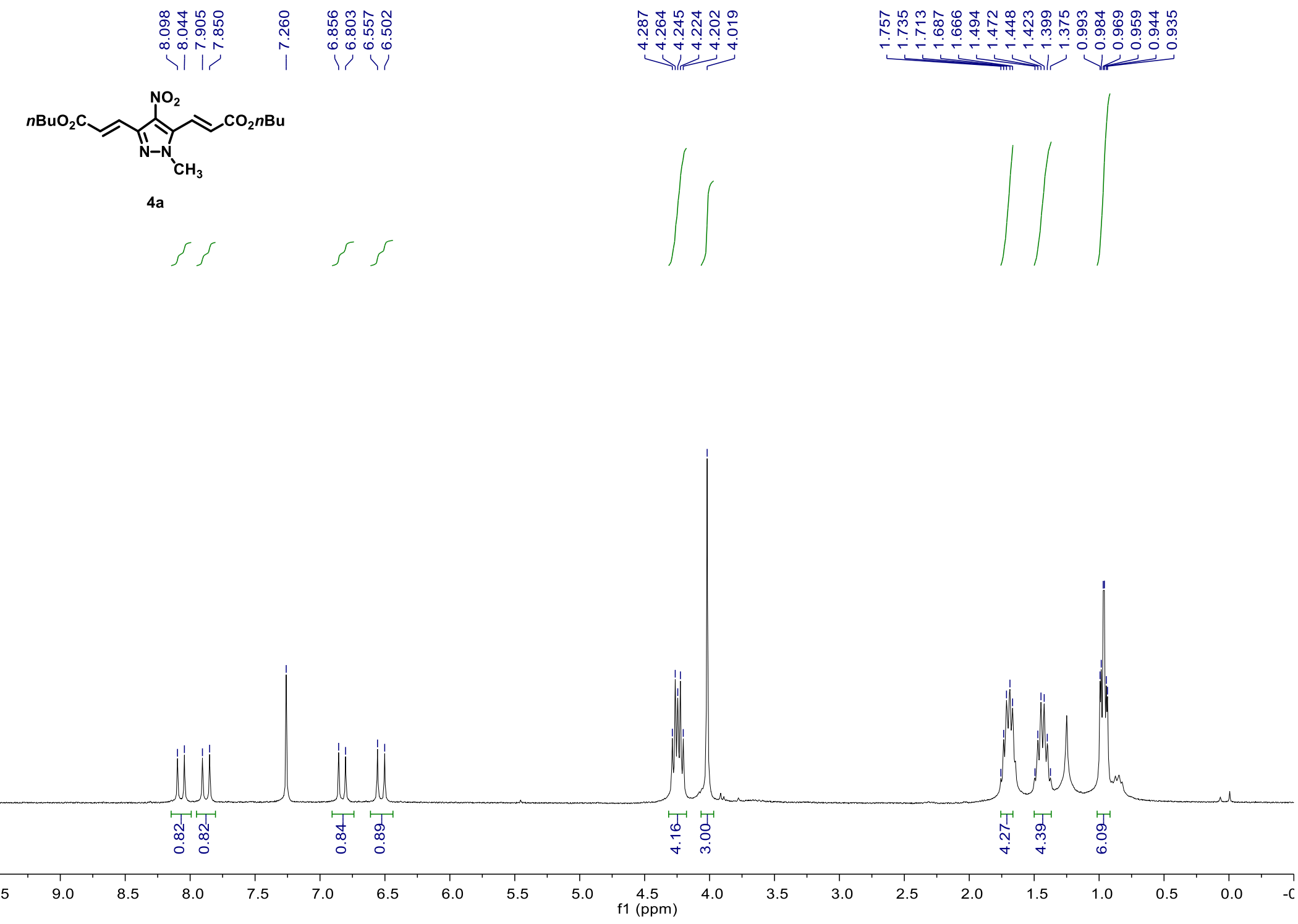




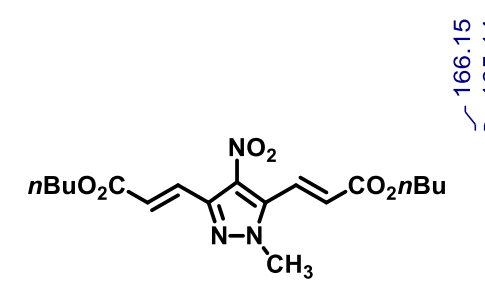

至

$4 a$

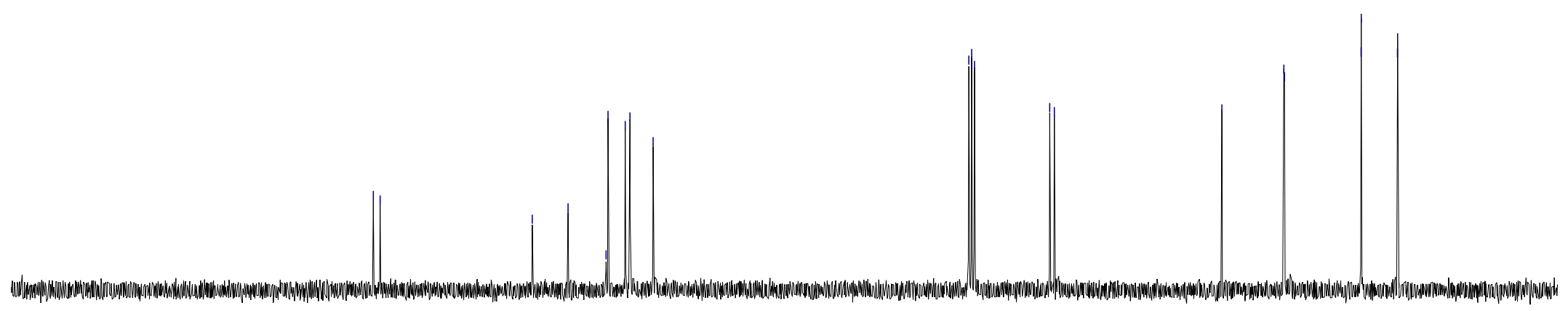

\begin{tabular}{lllllllllllllllllllllllll}
\hline & 210 & 200 & 190 & 180 & 170 & 160 & 150 & 140 & 130 & 120 & $\underset{\mathrm{f}}{110}(\mathrm{ppm})$ & 100 & 90 & 80 & 70 & 60 & 50 & 40 & 30 & 20 & 10 & 0 & -
\end{tabular}




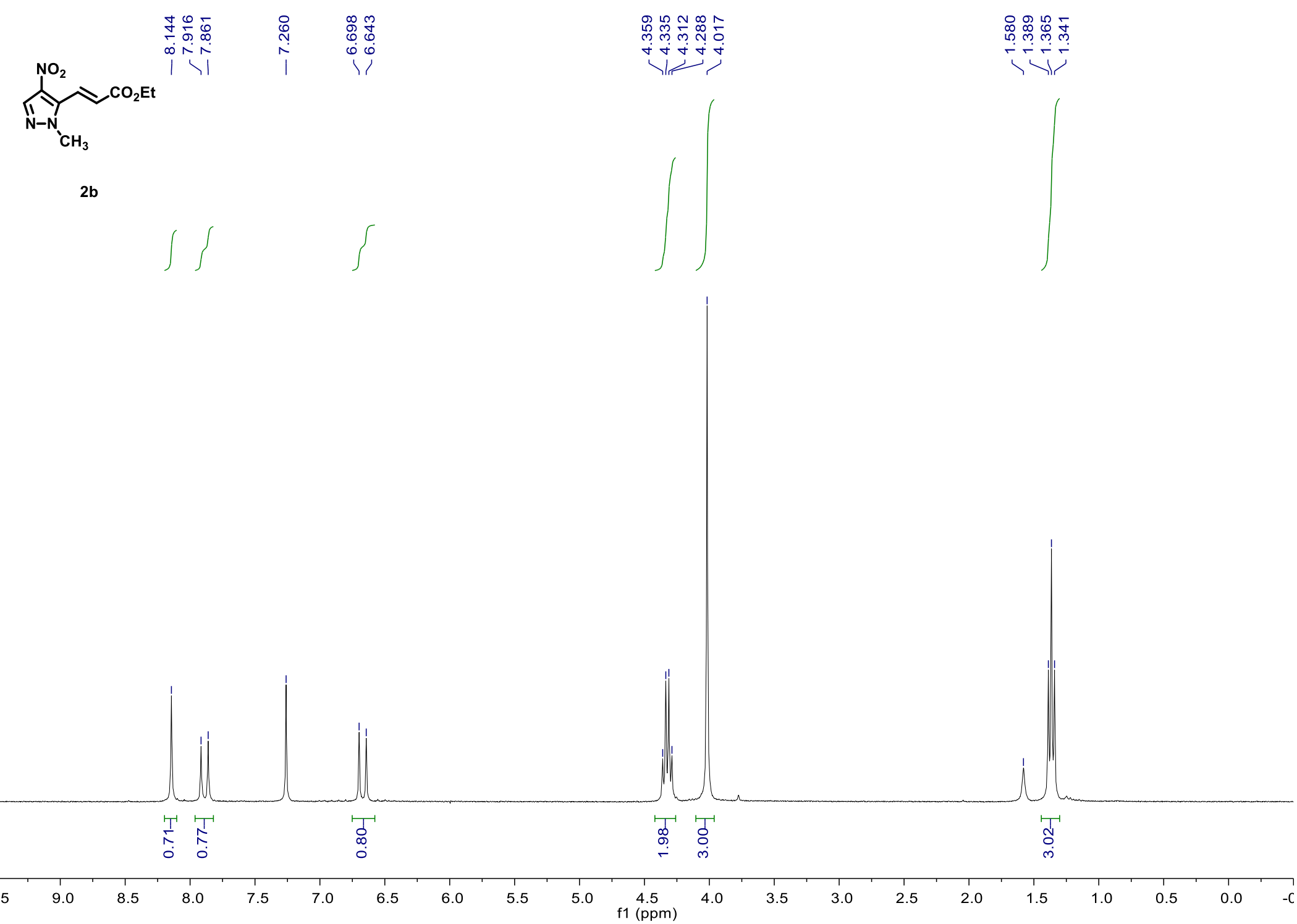




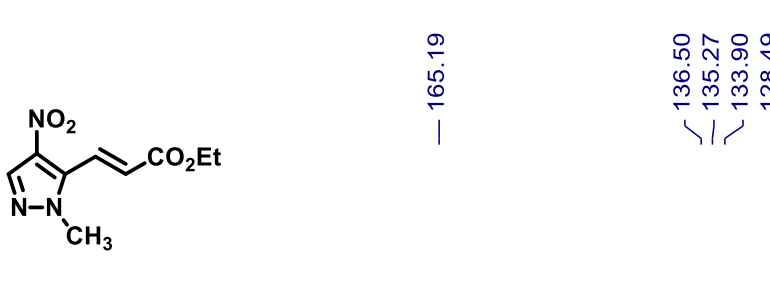

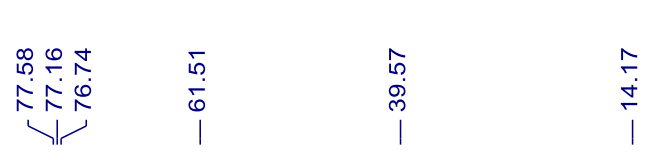

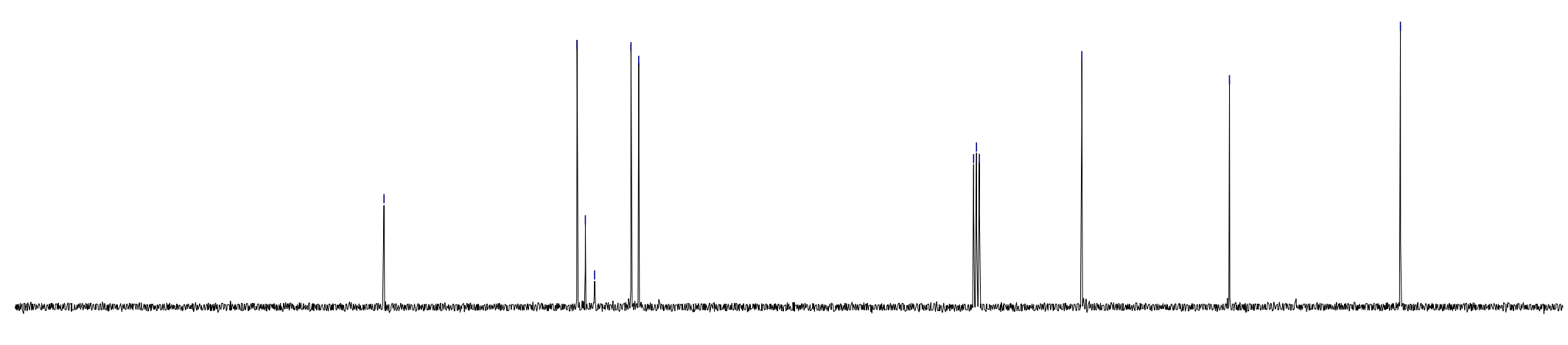




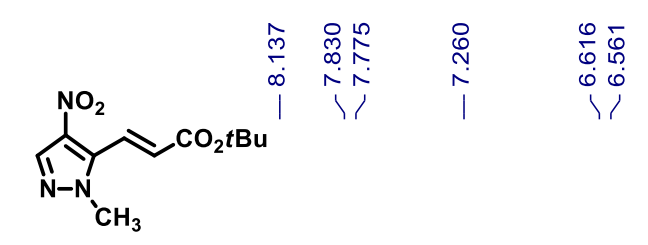

$2 c$

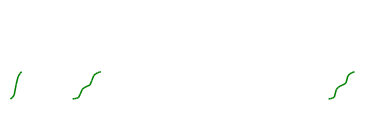

$\int$

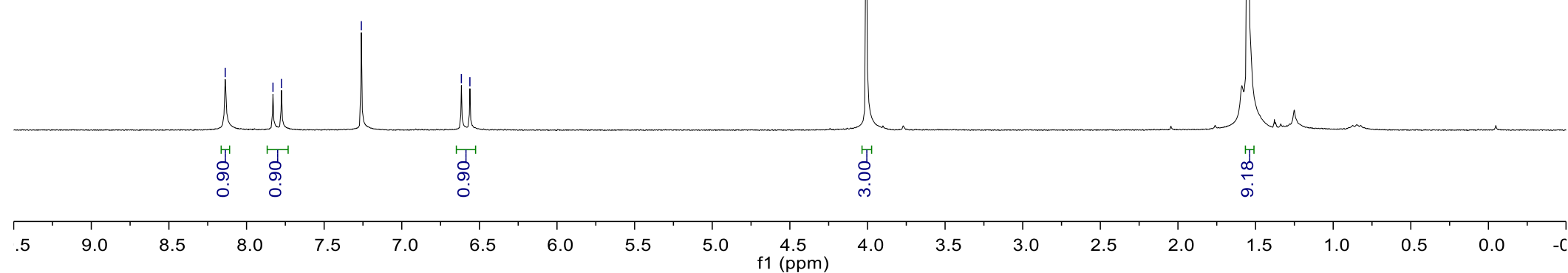




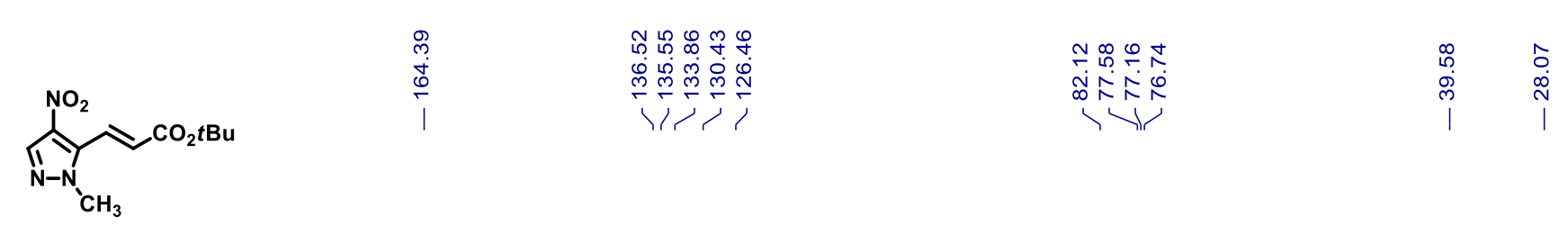

2c

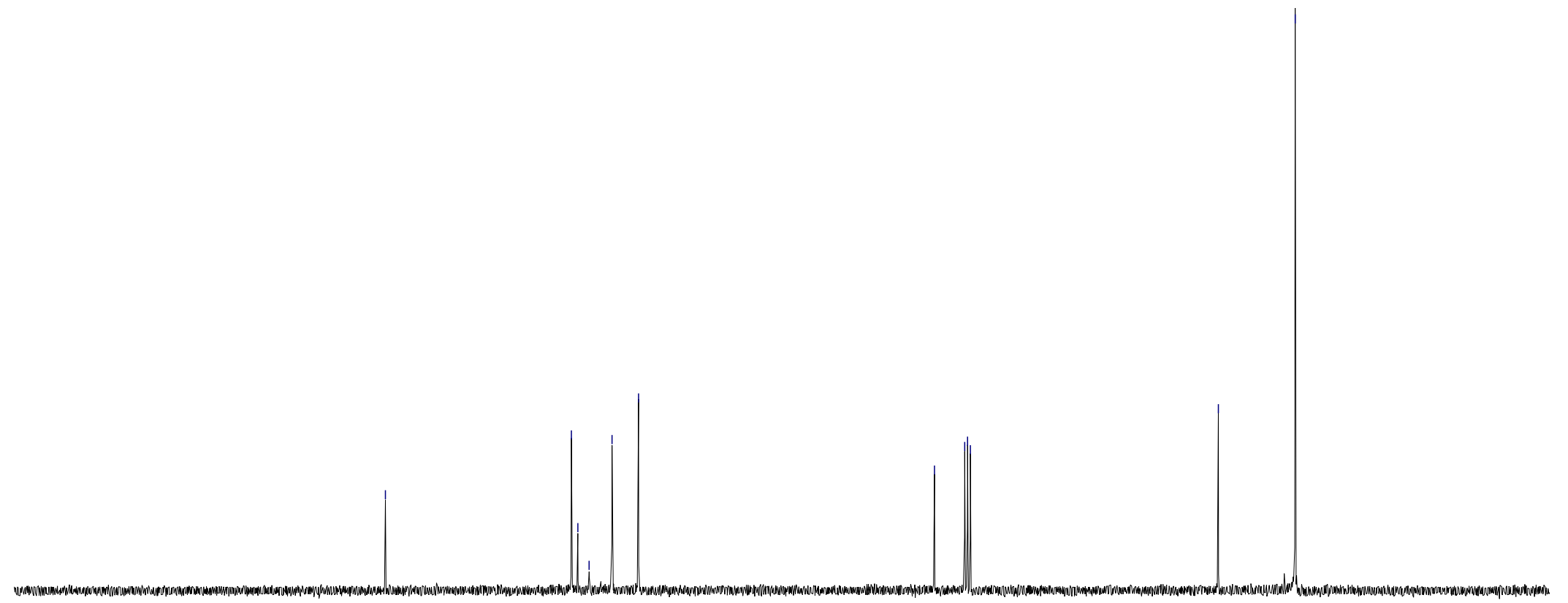

$\begin{array}{lllllllllllllllllllllllll}210 & 200 & 190 & 180 & 170 & 160 & 150 & 140 & 130 & 120 & 110 & 100 & 90 & 80 & 70 & 60 & 50 & 40 & 30 & 20 & 10 & 0 & -\end{array}$ 

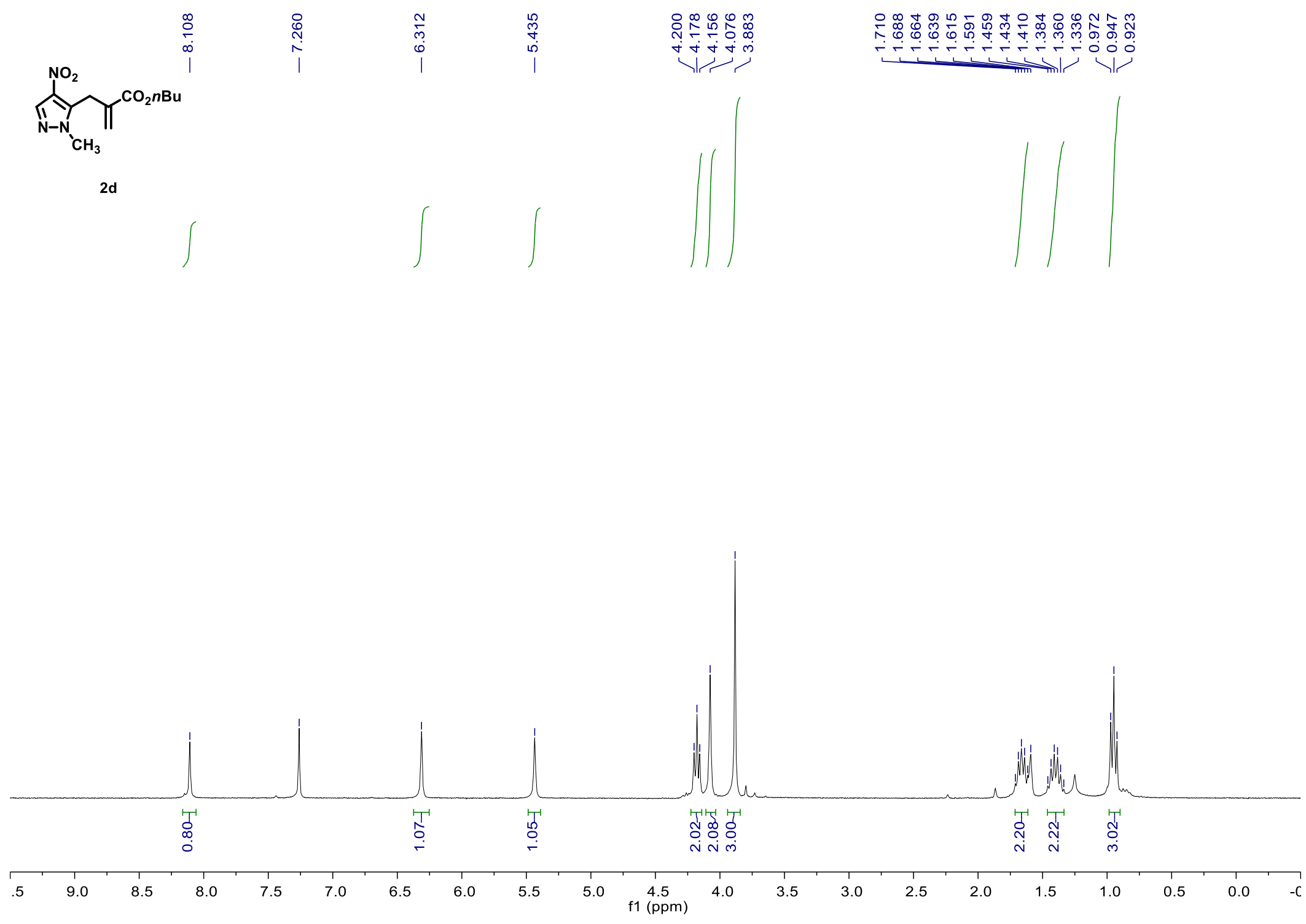


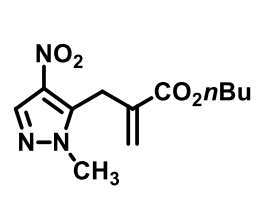

㼭影

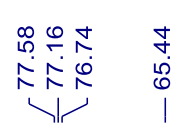

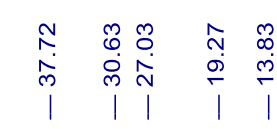

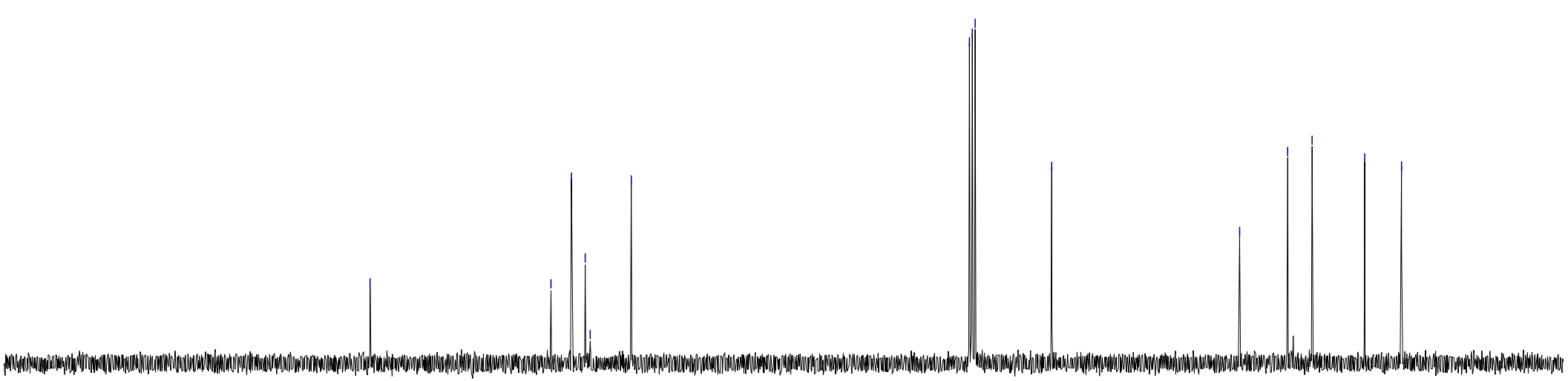

S14 

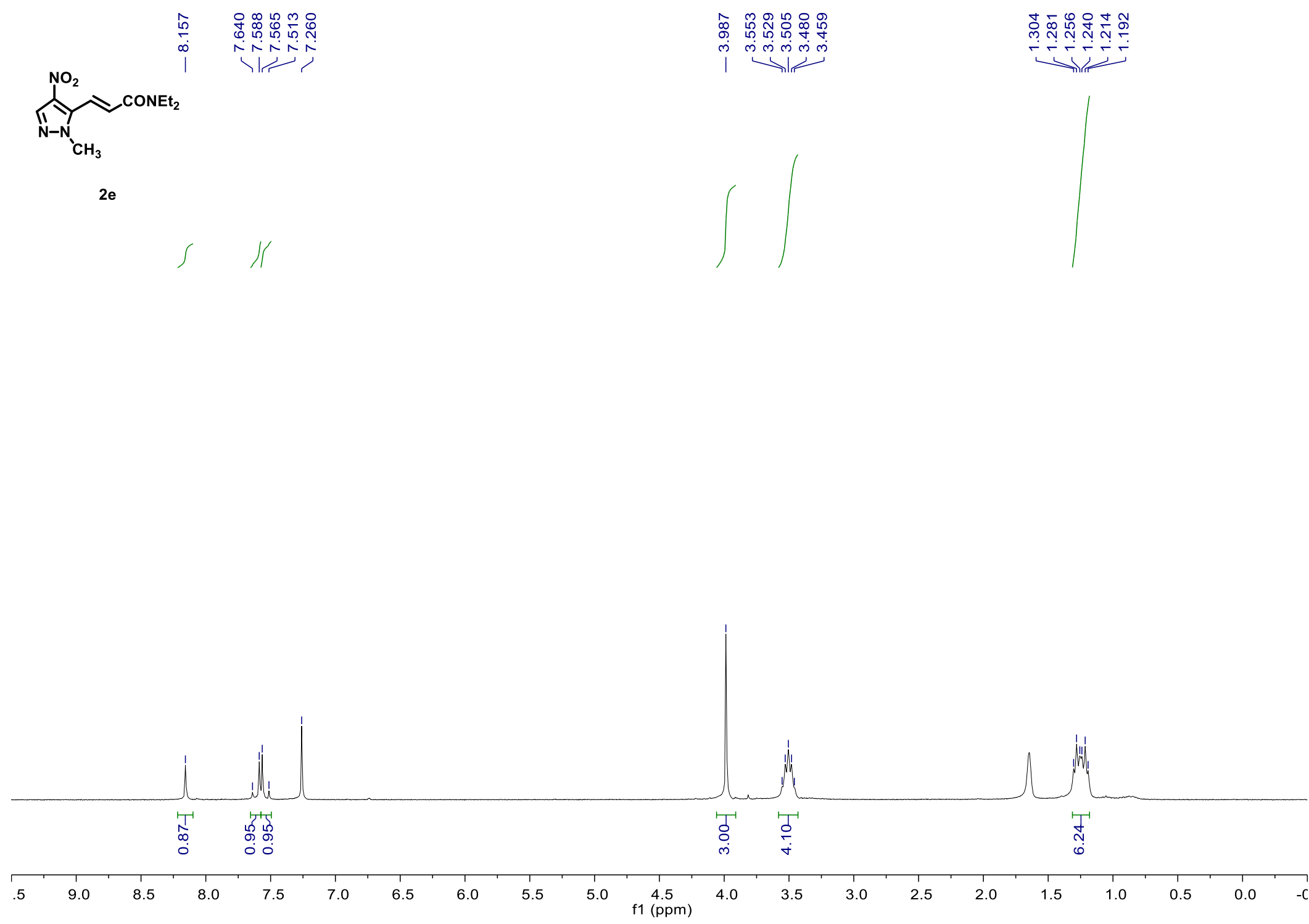


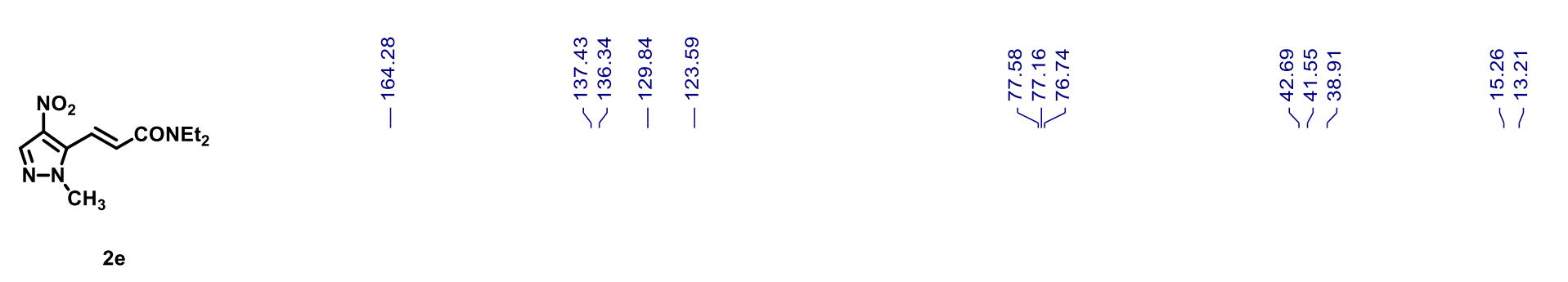

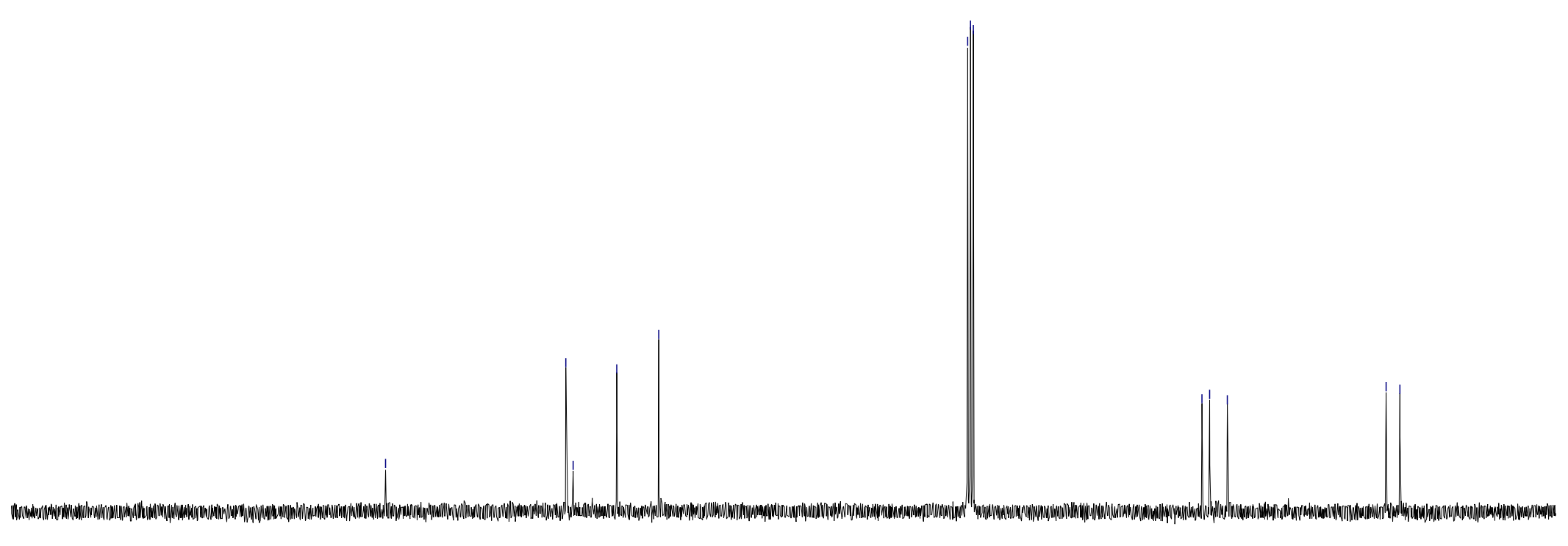

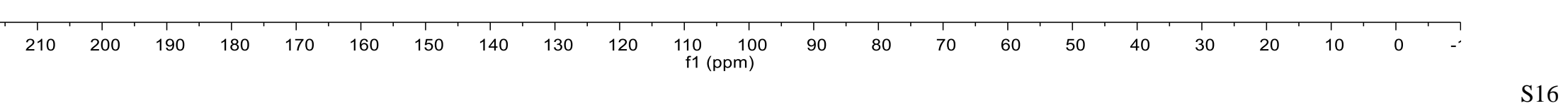



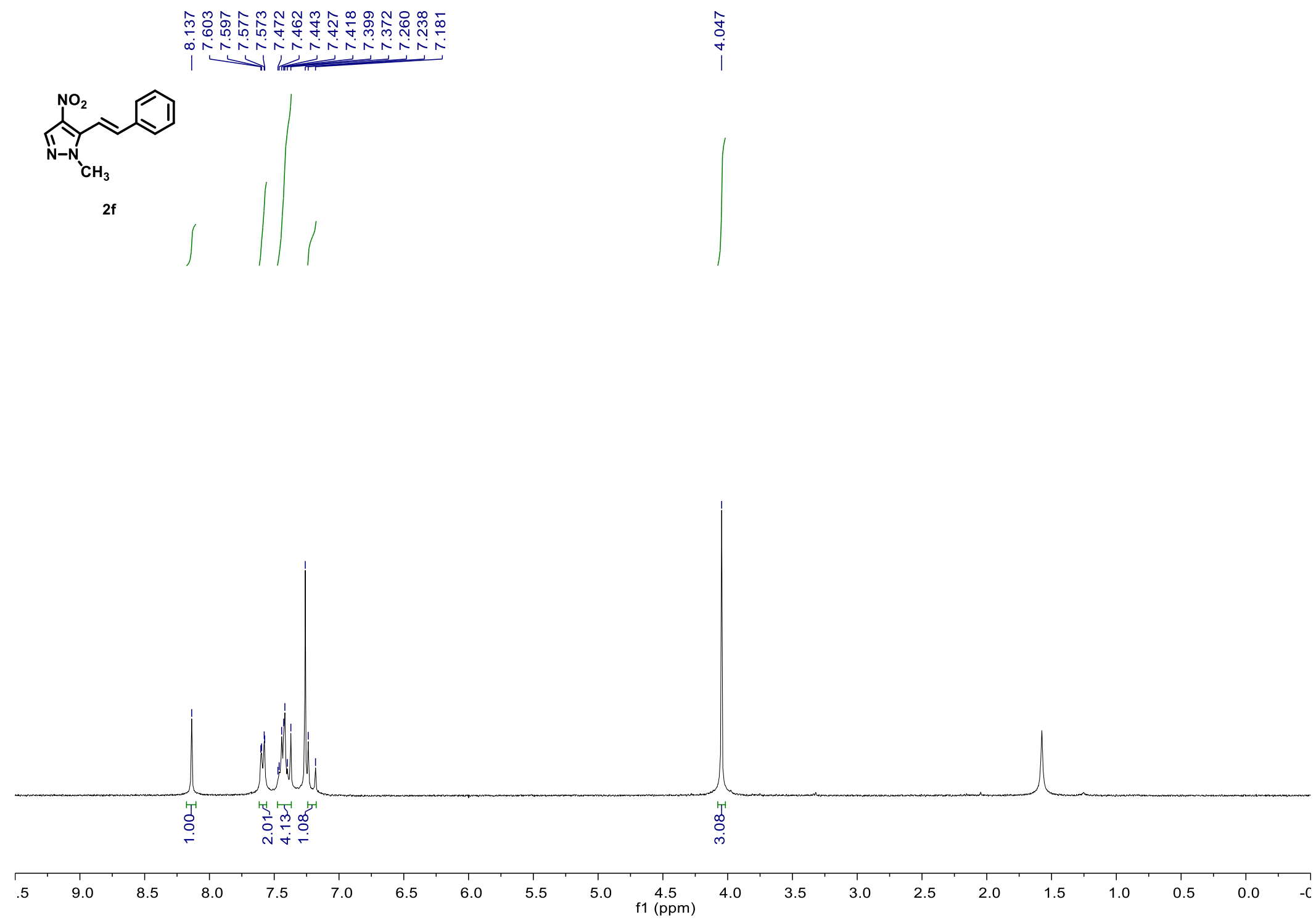


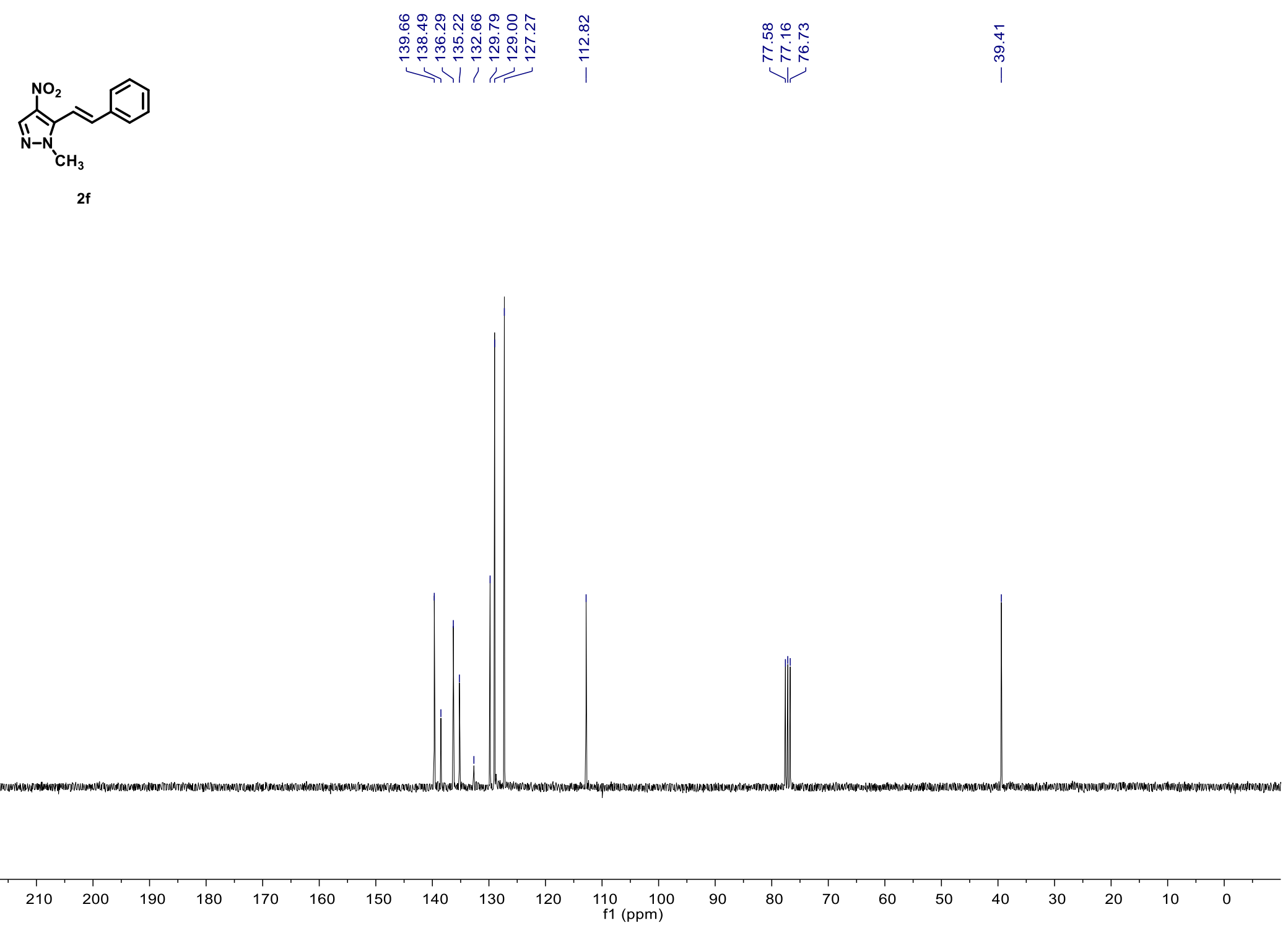




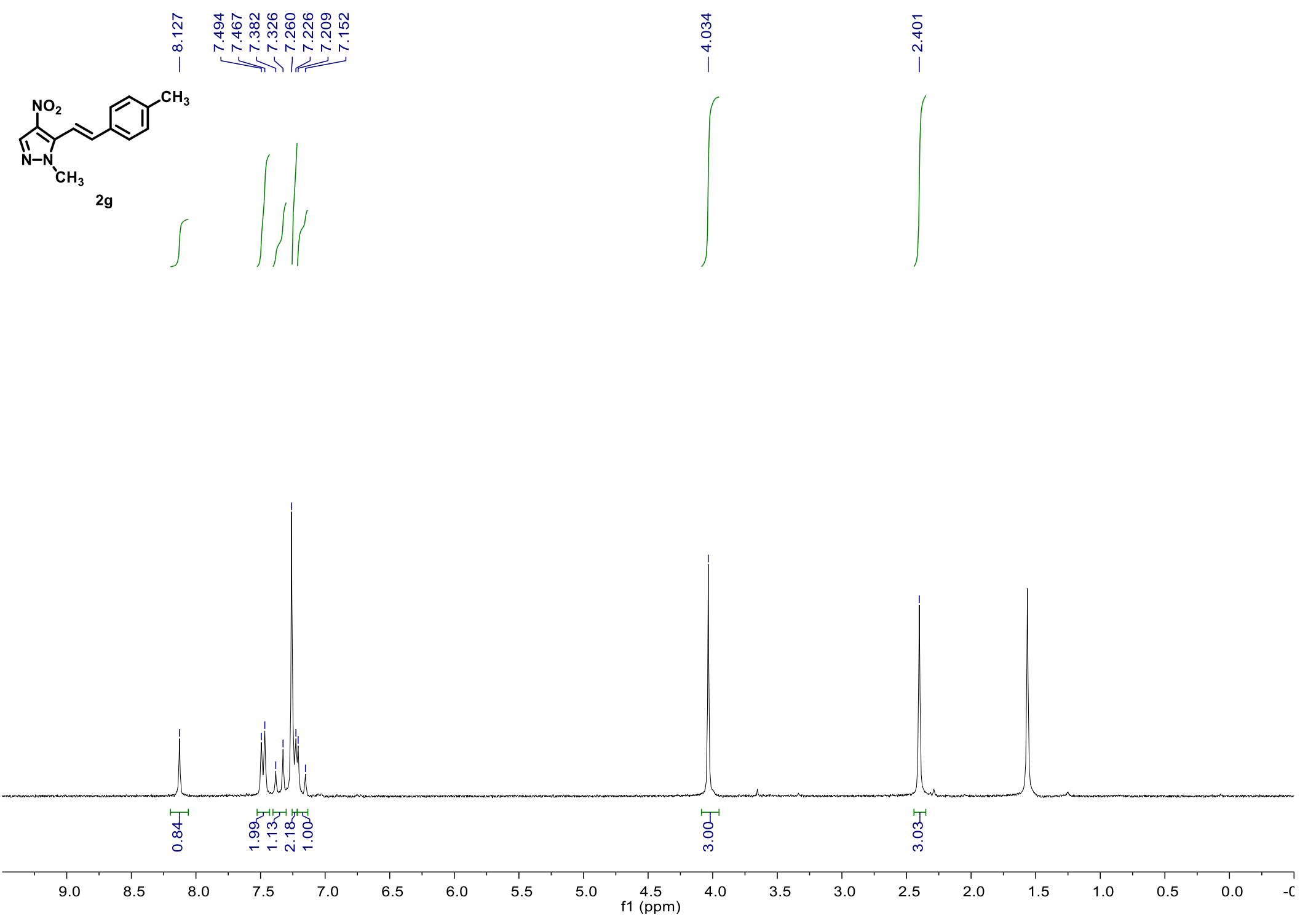




$$
\text { s. }
$$



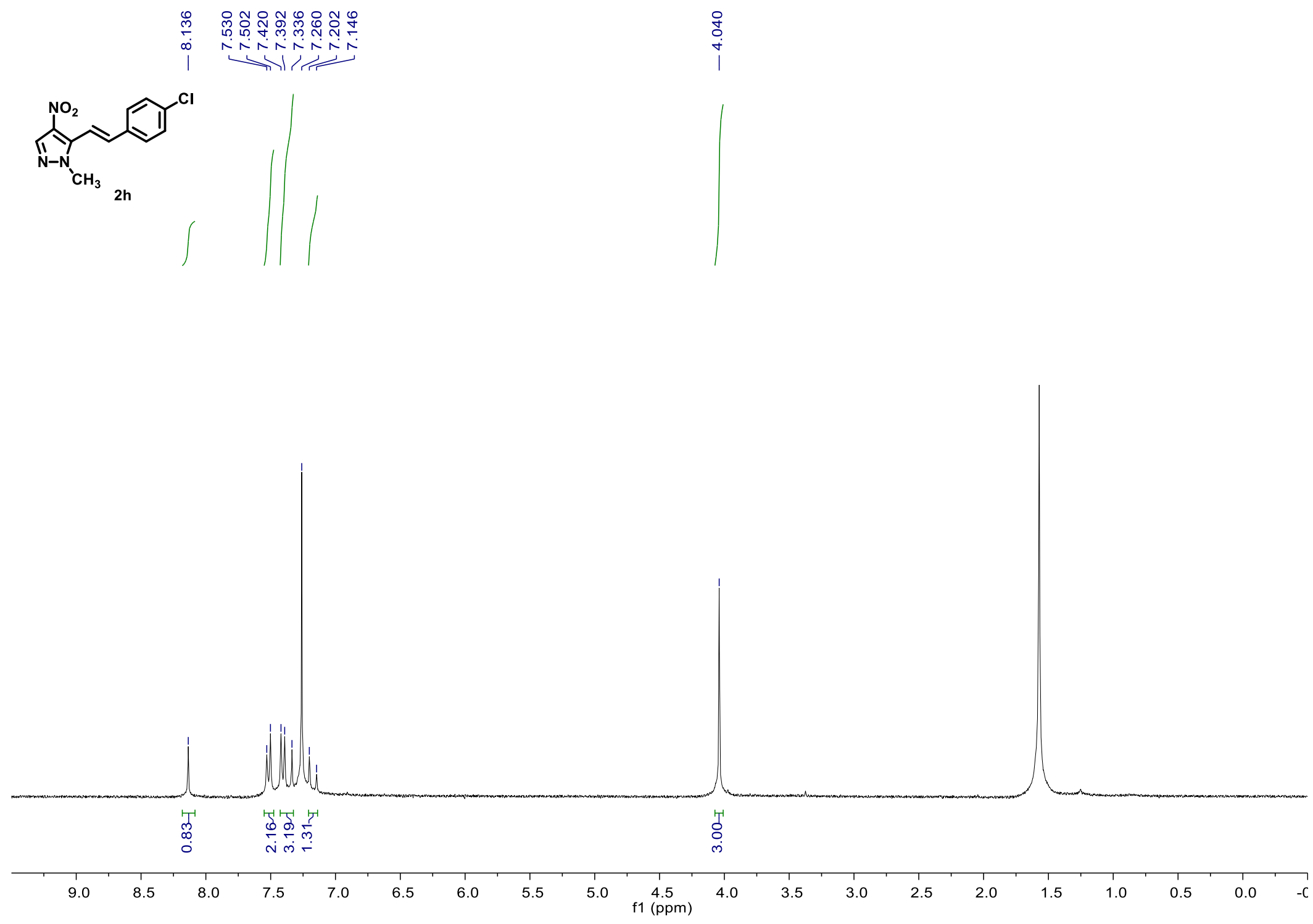


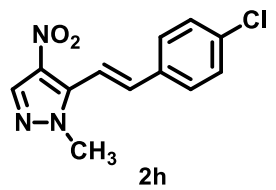

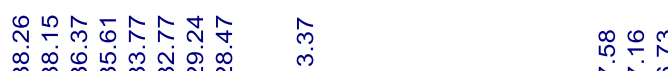

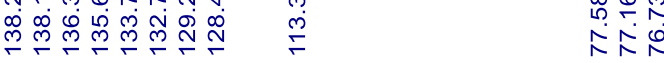

$\stackrel{0}{\stackrel{0}{0}}$

们垈

둔

ले

$\mathrm{CH}_{3}$

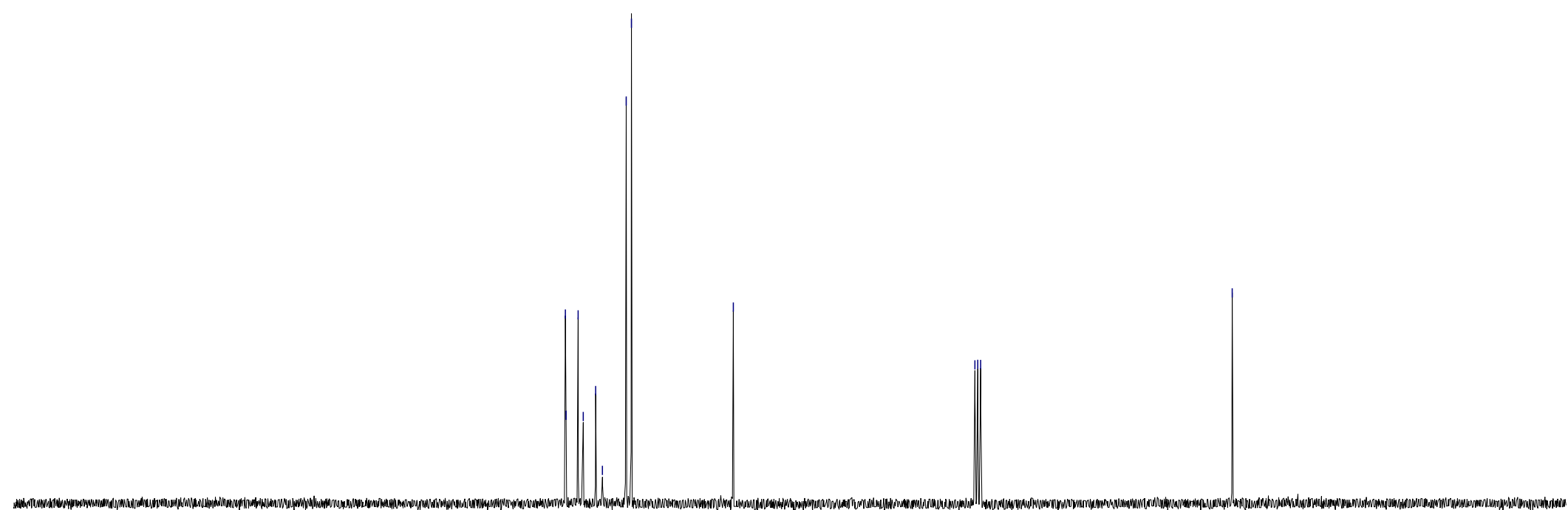

$\begin{array}{lllllllllllllllllllllllll}210 & 200 & 190 & 180 & 170 & 160 & 150 & 140 & 130 & 120 & 110 & 100 & 90 & 80 & 70 & 60 & 50 & 40 & 30 & 20 & 10 & 0 & -\end{array}$ 

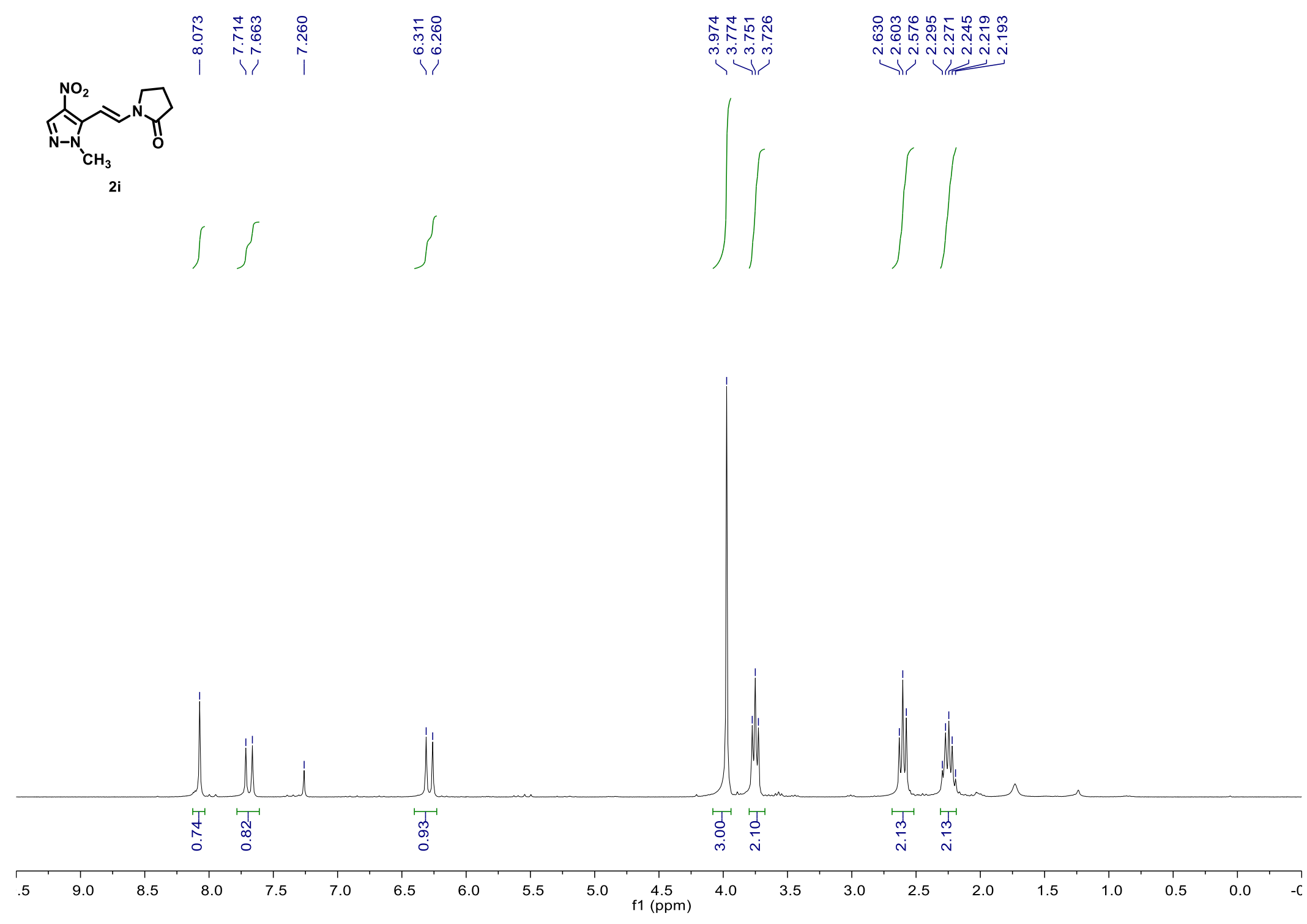


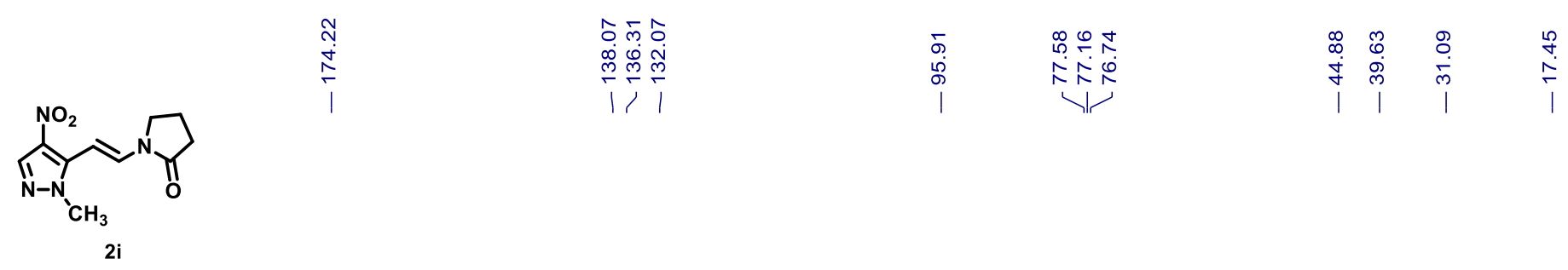

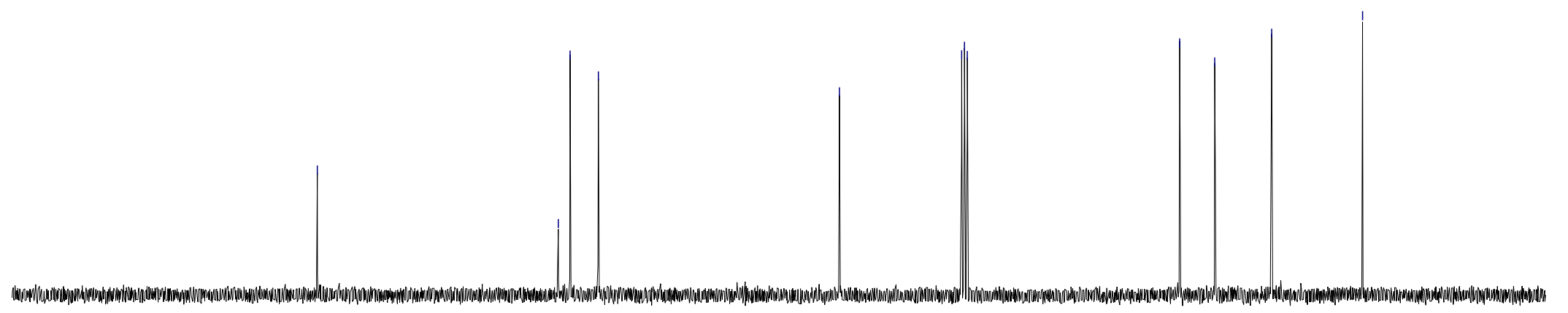

$\begin{array}{lllllllllll}210 & 200 & 190 & 180 & 170 & 160 & 150 & 140 & 130 & 120 & \begin{array}{c}110 \\ \mathrm{f} 1(\mathrm{ppm})\end{array}\end{array}$ 


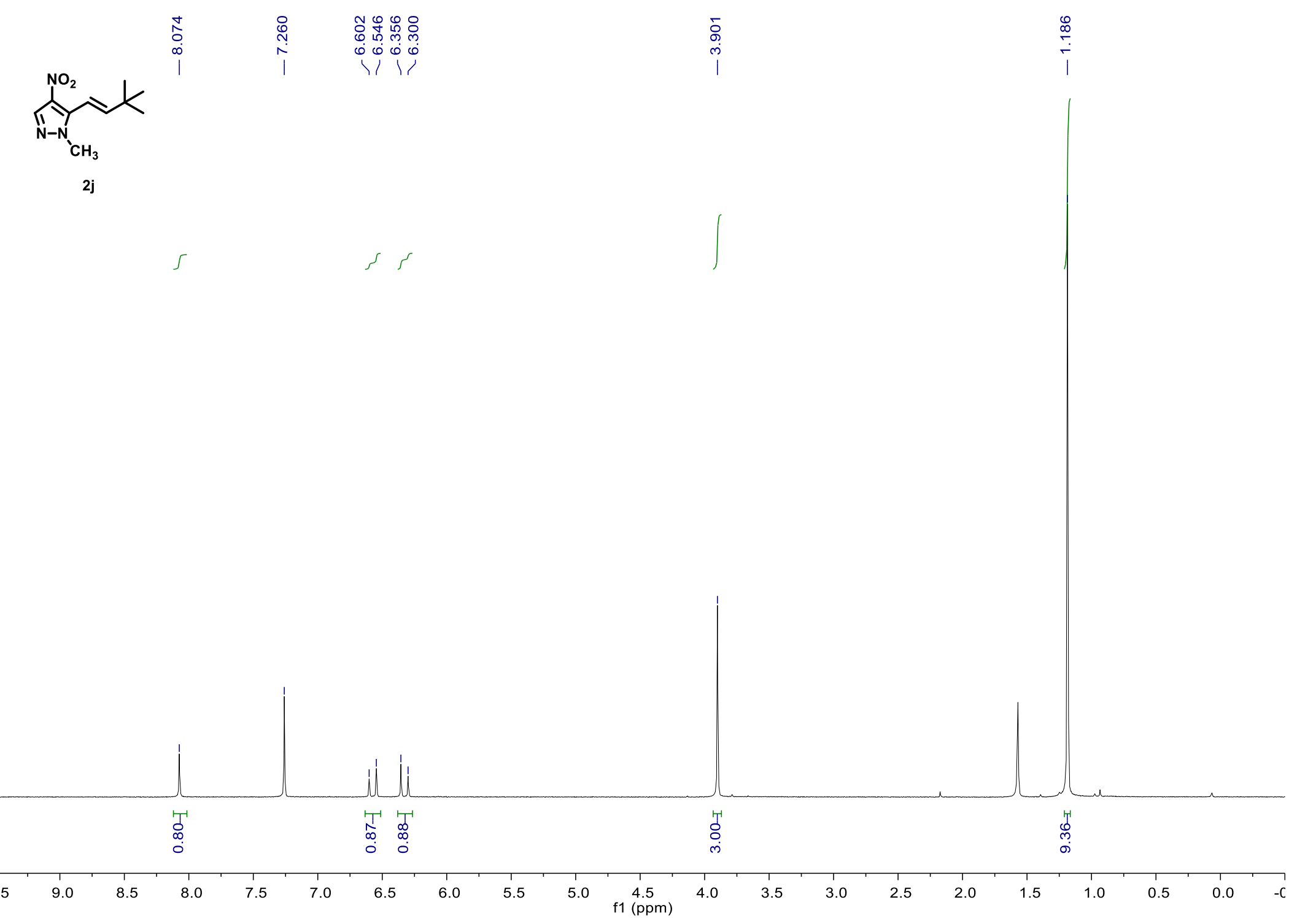




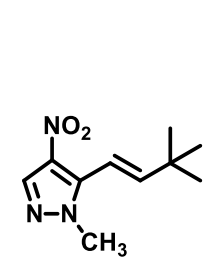

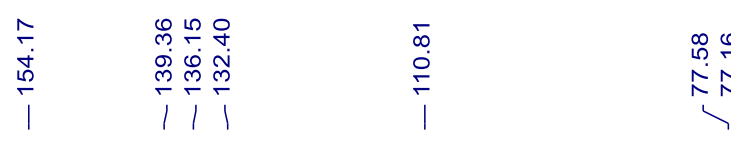

$\sqrt{5}$

$\stackrel{100}{10}$

品

$\mathrm{CH}_{3}$

2.

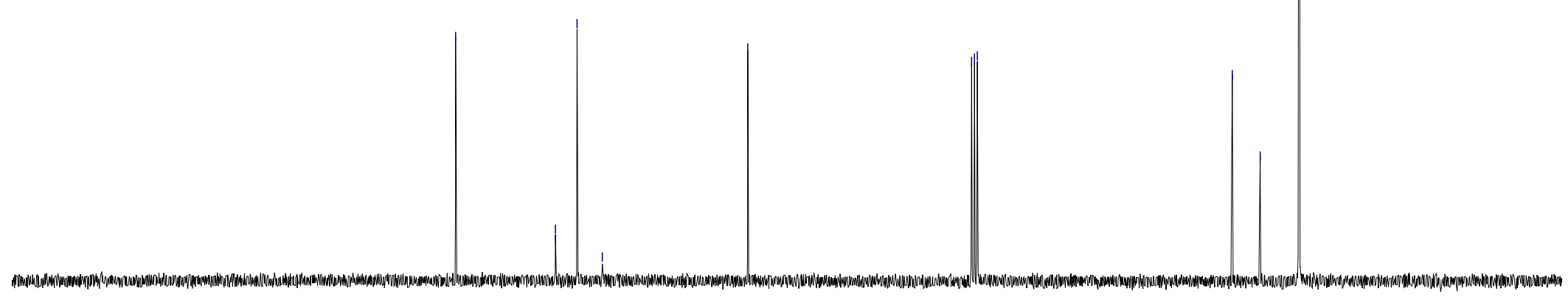

$\begin{array}{lllllllllll}210 & 200 & 190 & 180 & 170 & 160 & 150 & 140 & 130 & 120 & \begin{array}{c}110 \\ \mathrm{f} 1(\mathrm{ppm})\end{array}\end{array}$ 
$\sum_{\mathrm{N}-\mathrm{N}}^{\mathrm{NO}}$

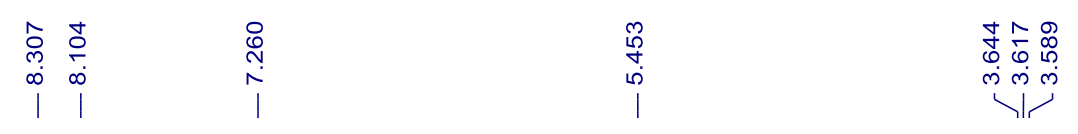

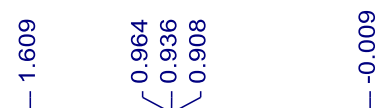

Sa

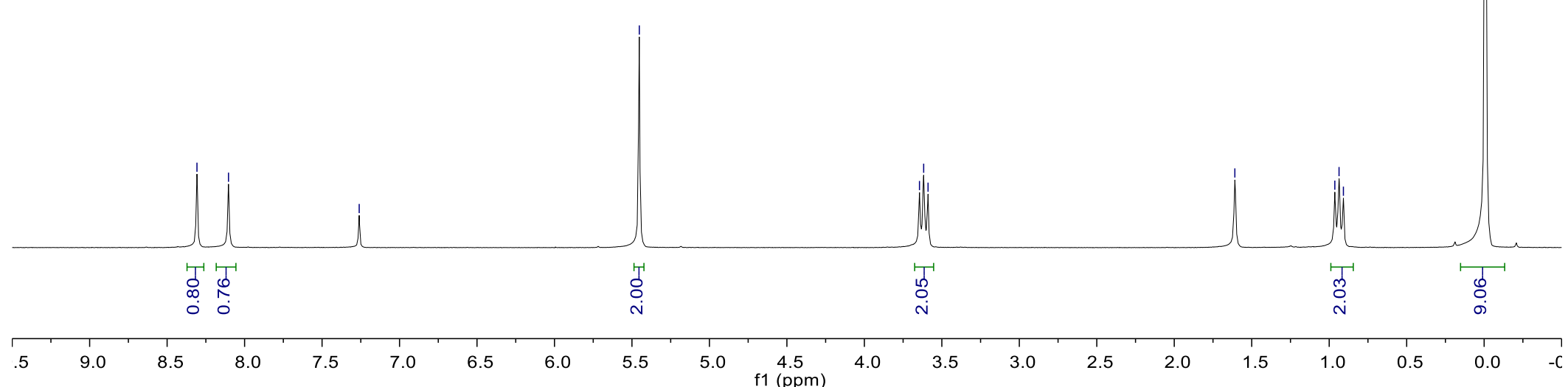



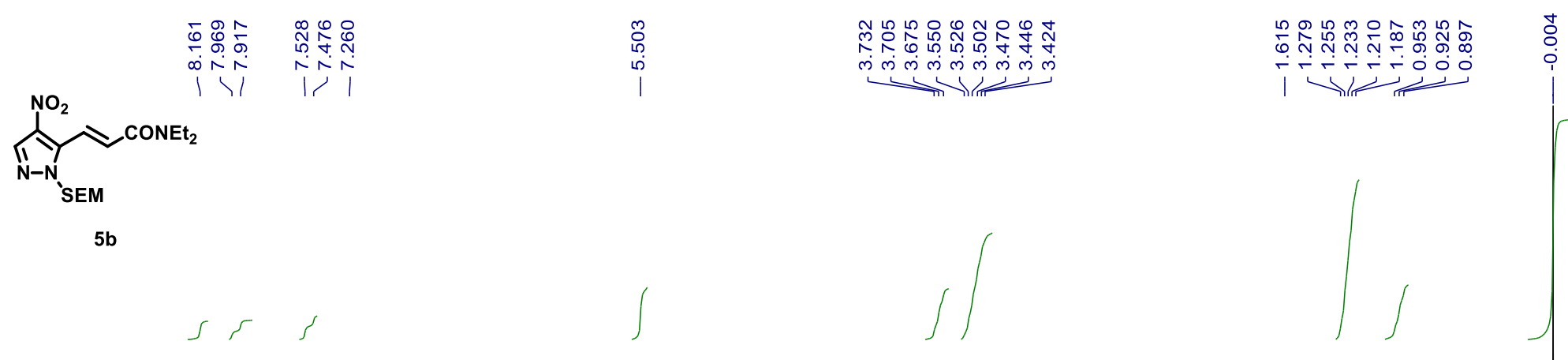

$5 b$
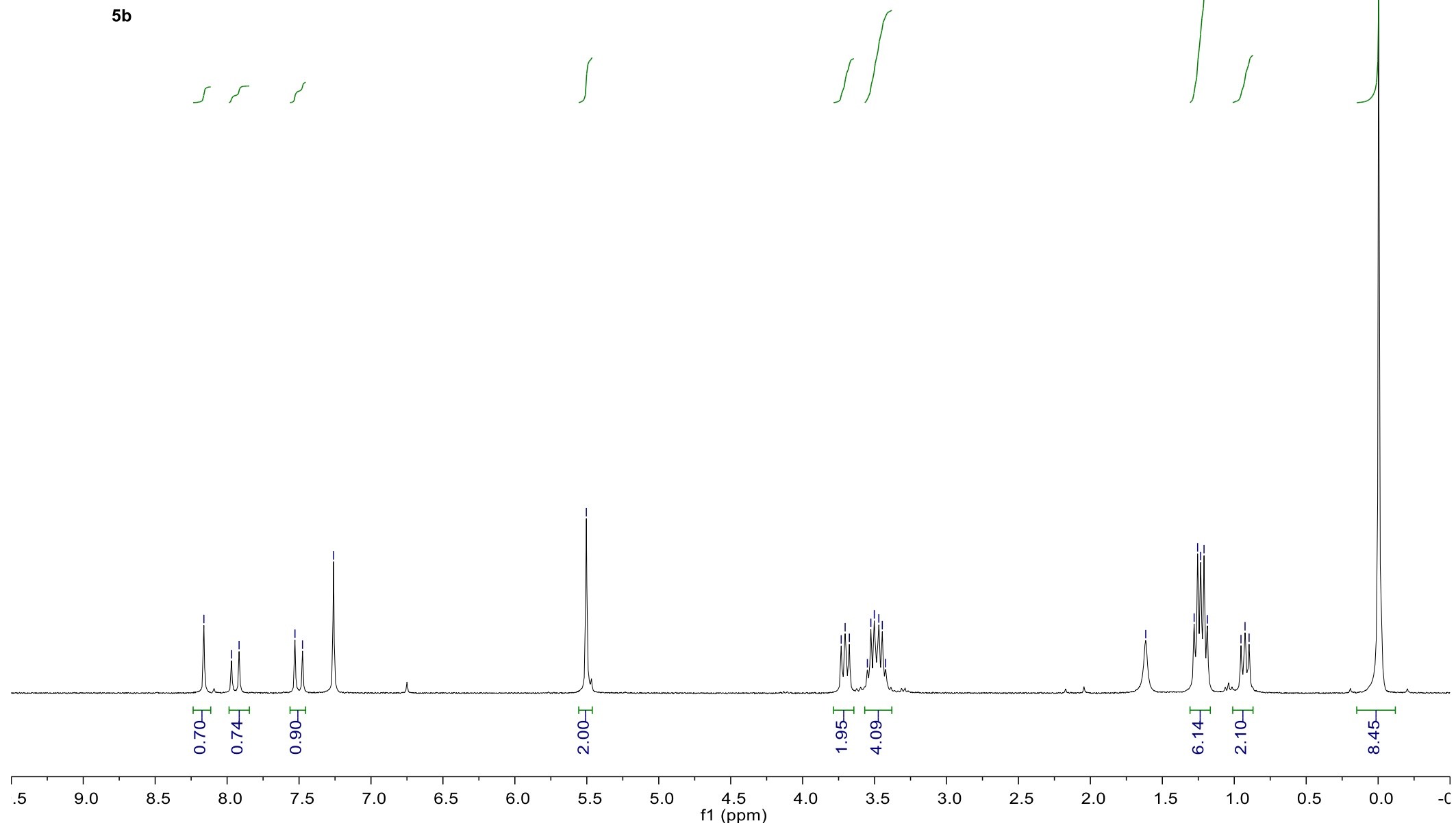

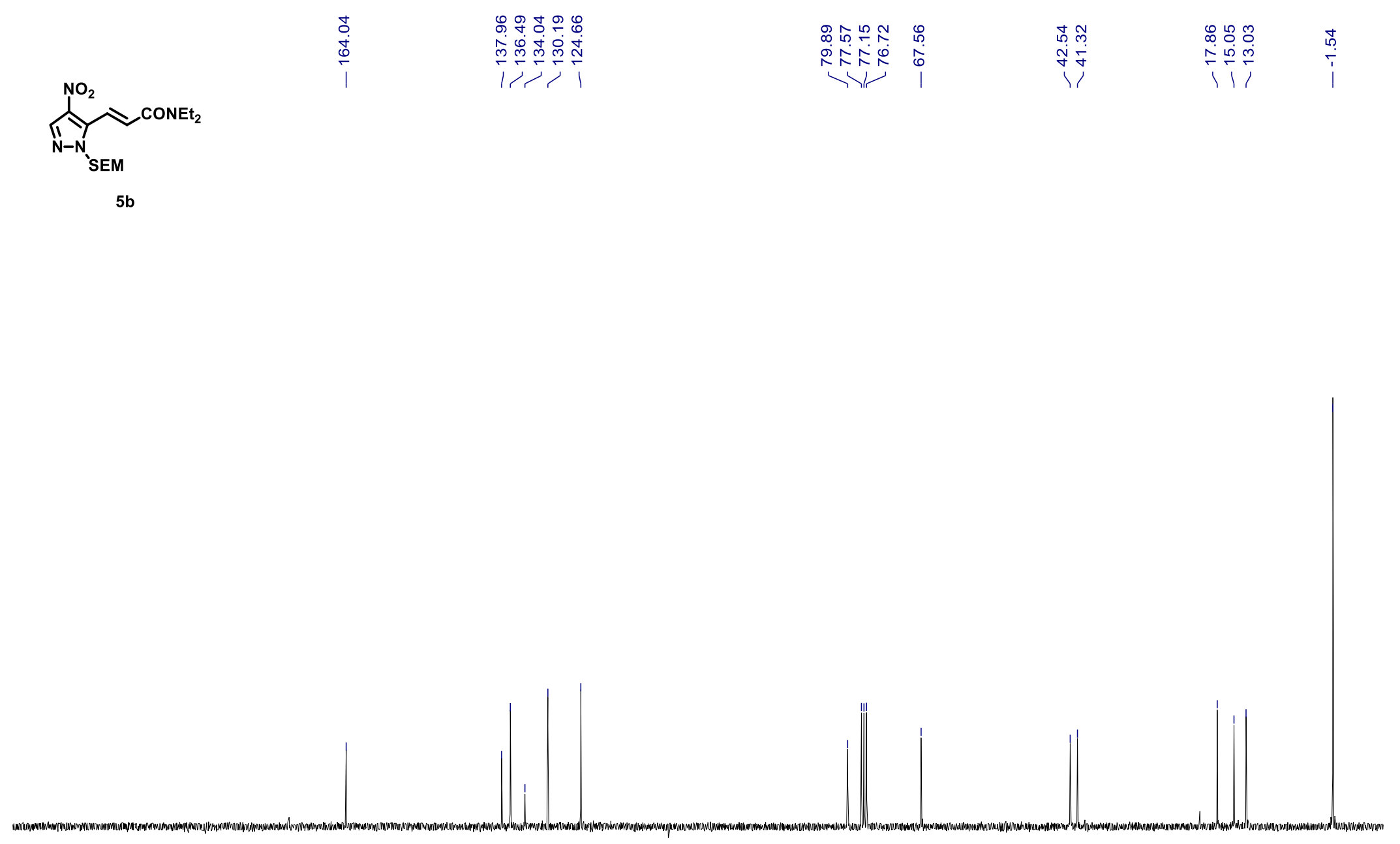

$\begin{array}{lllllllllllllllllllllllllllll} & 210 & 200 & 190 & 180 & 170 & 160 & 150 & 140 & 130 & 120 & 110 & 100 & 90 & 80 & 70 & 60 & 50 & 40 & 30 & 20 & 10 & 0 & -\end{array}$ 

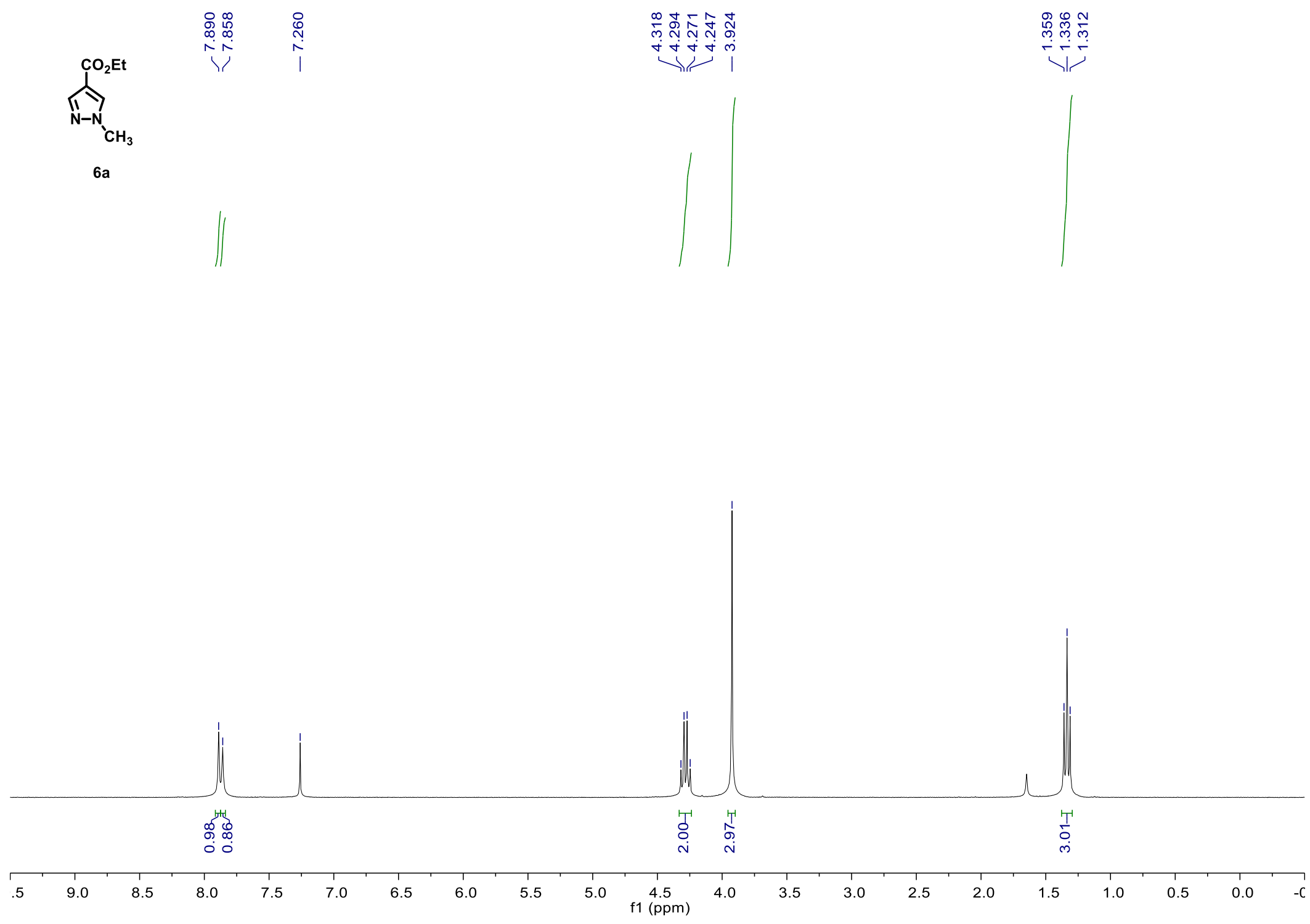

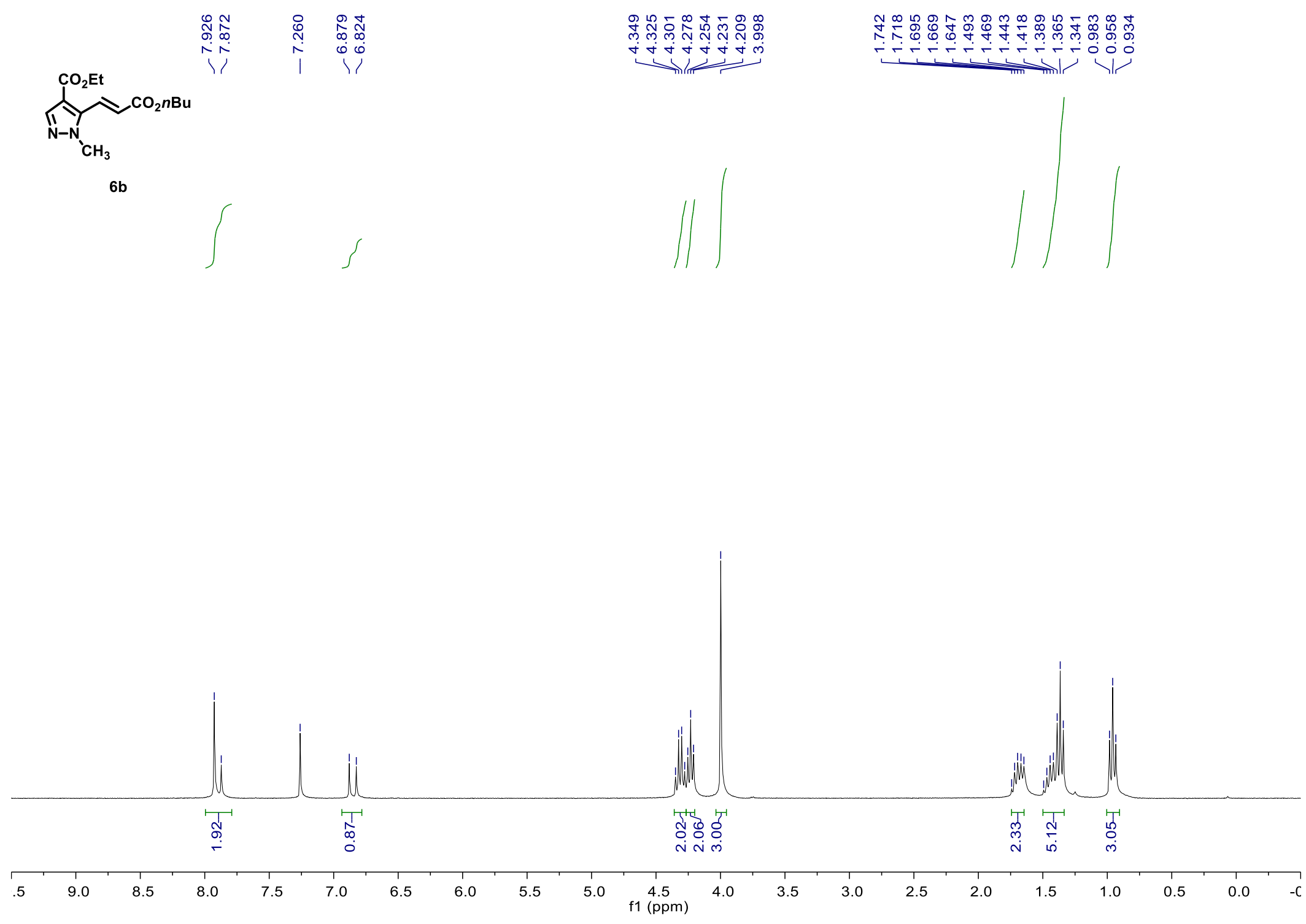

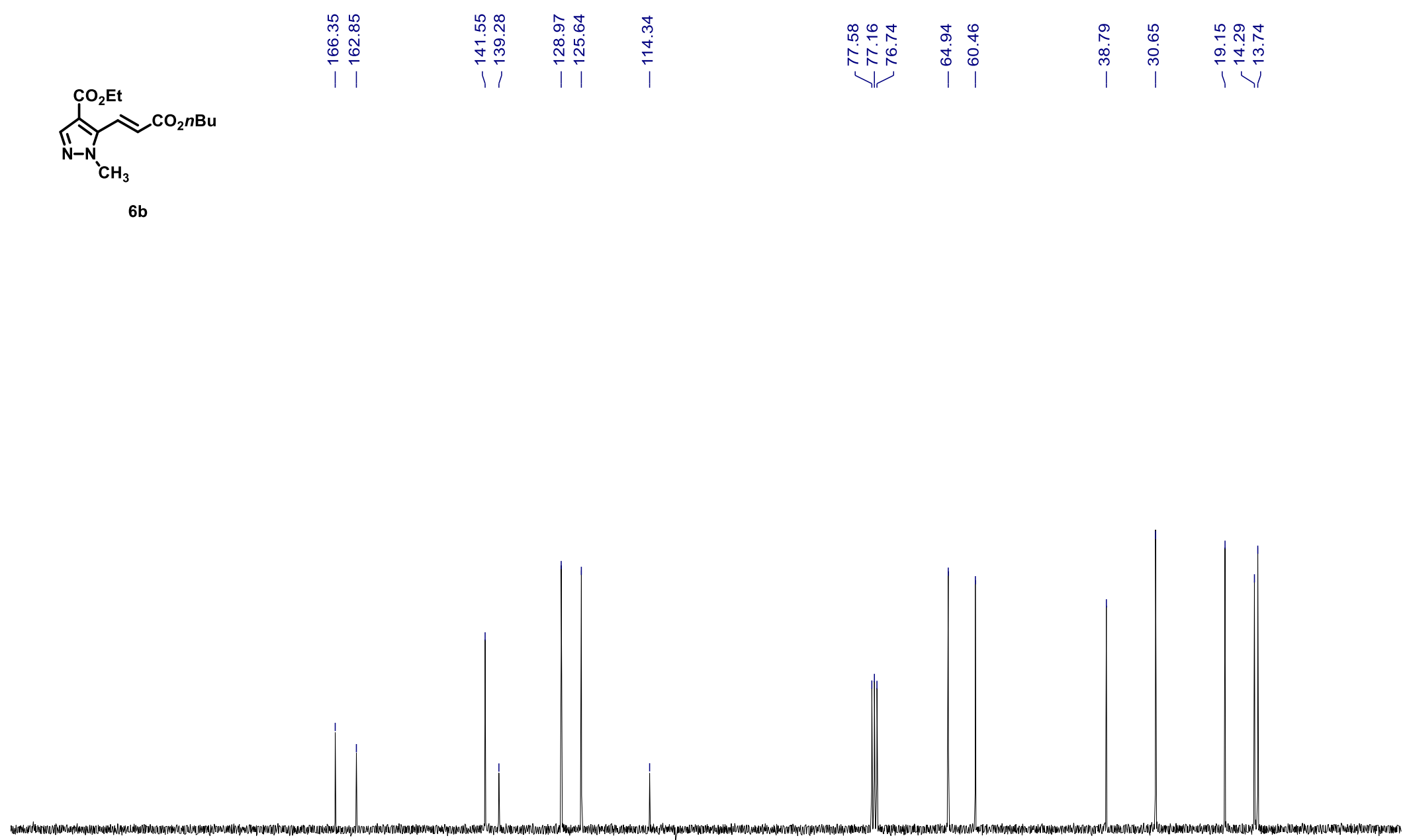

$\begin{array}{lllllllllll}210 & 200 & 190 & 180 & 170 & 160 & 150 & 140 & 130 & 120 & \begin{array}{c}110 \\ \mathrm{f} 1(\mathrm{ppm})\end{array}\end{array}$ 
$\overbrace{\mathrm{N}-\mathrm{N} \mathrm{CH}_{3}}^{\mathrm{OOCH}_{3}}$

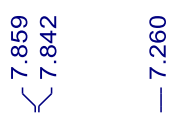

$\underset{\substack{\sigma \\ \infty}}{\infty}$

$\underset{\substack{\text { N } \\ \text { I }}}{ }$

7
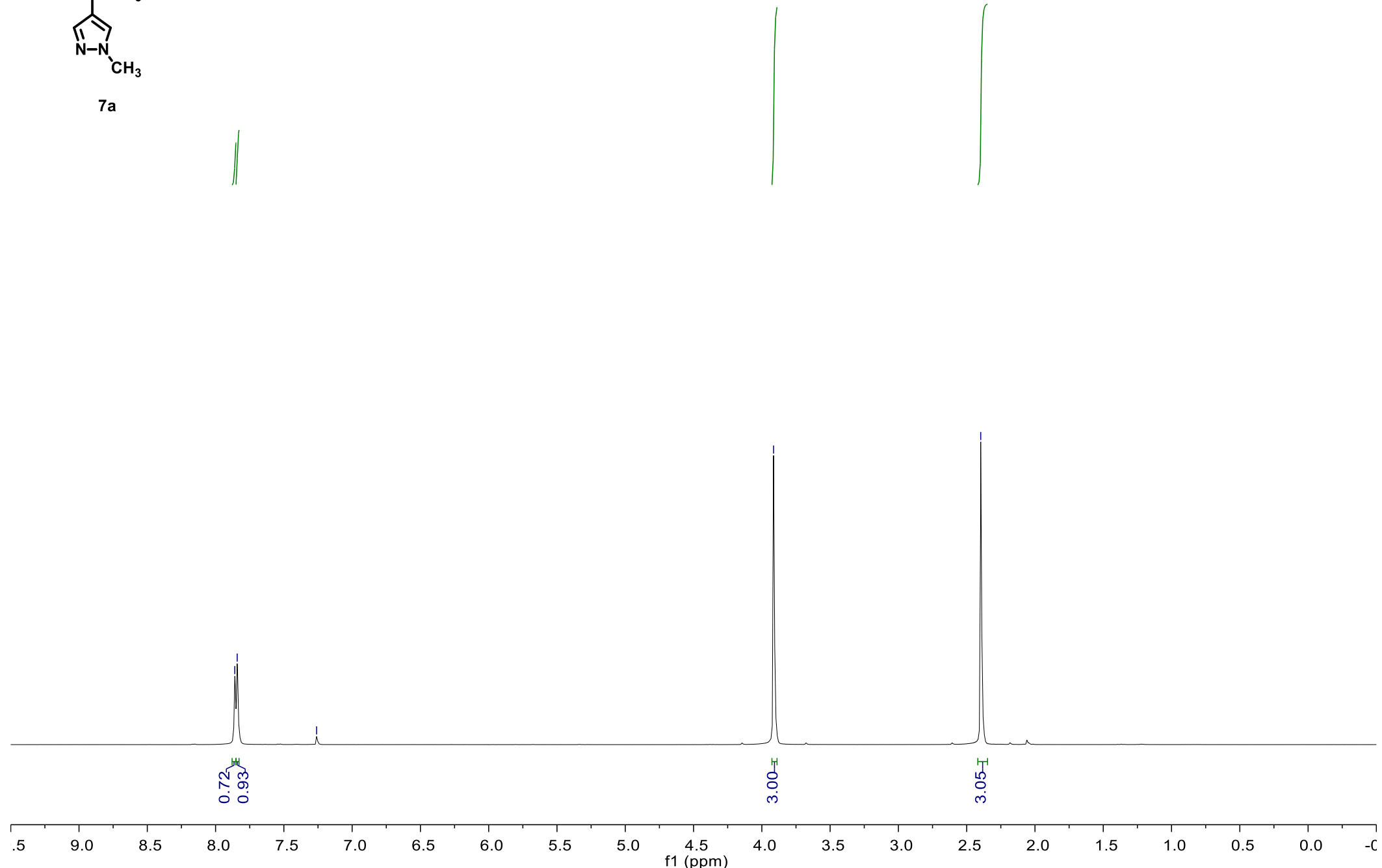

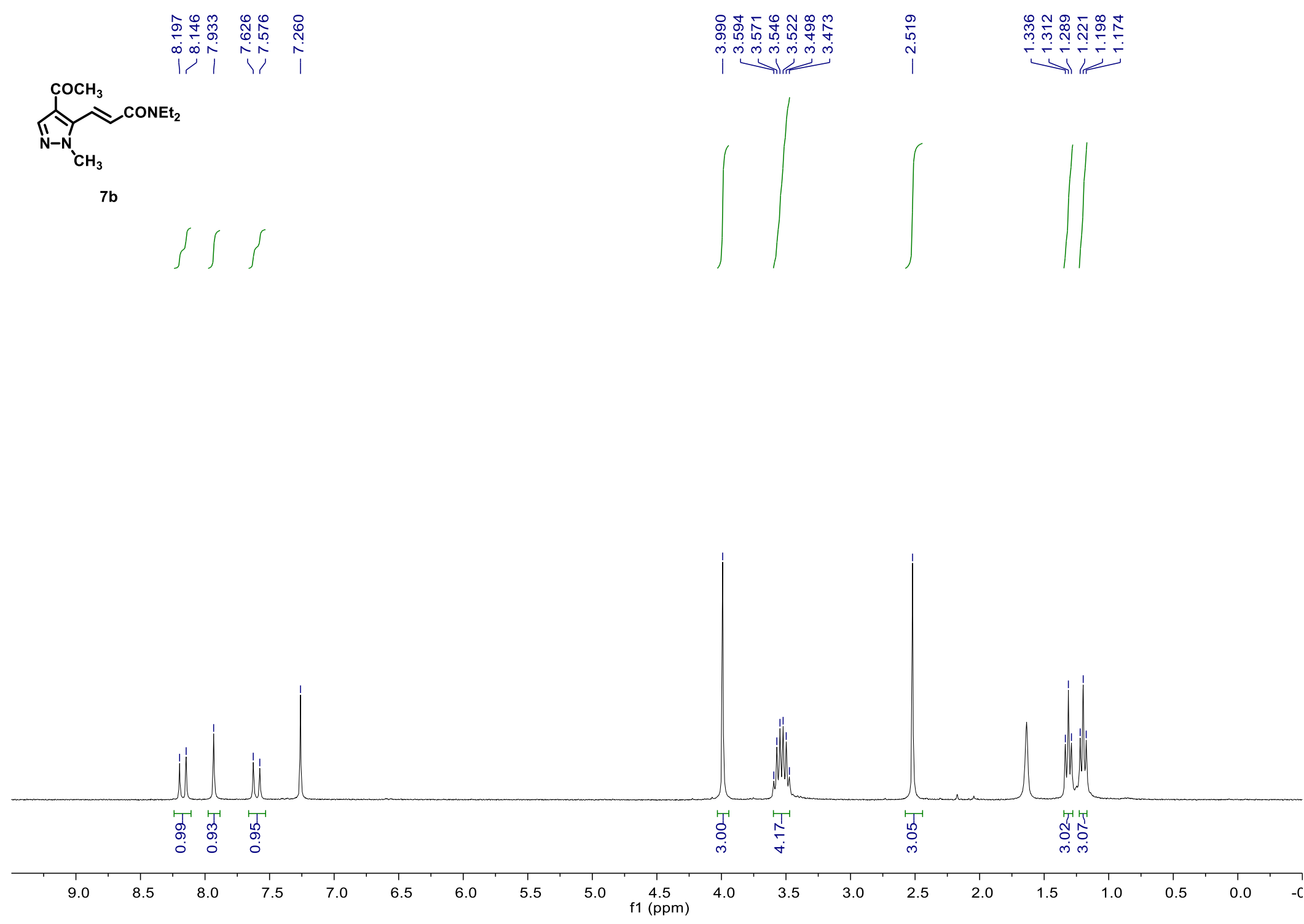

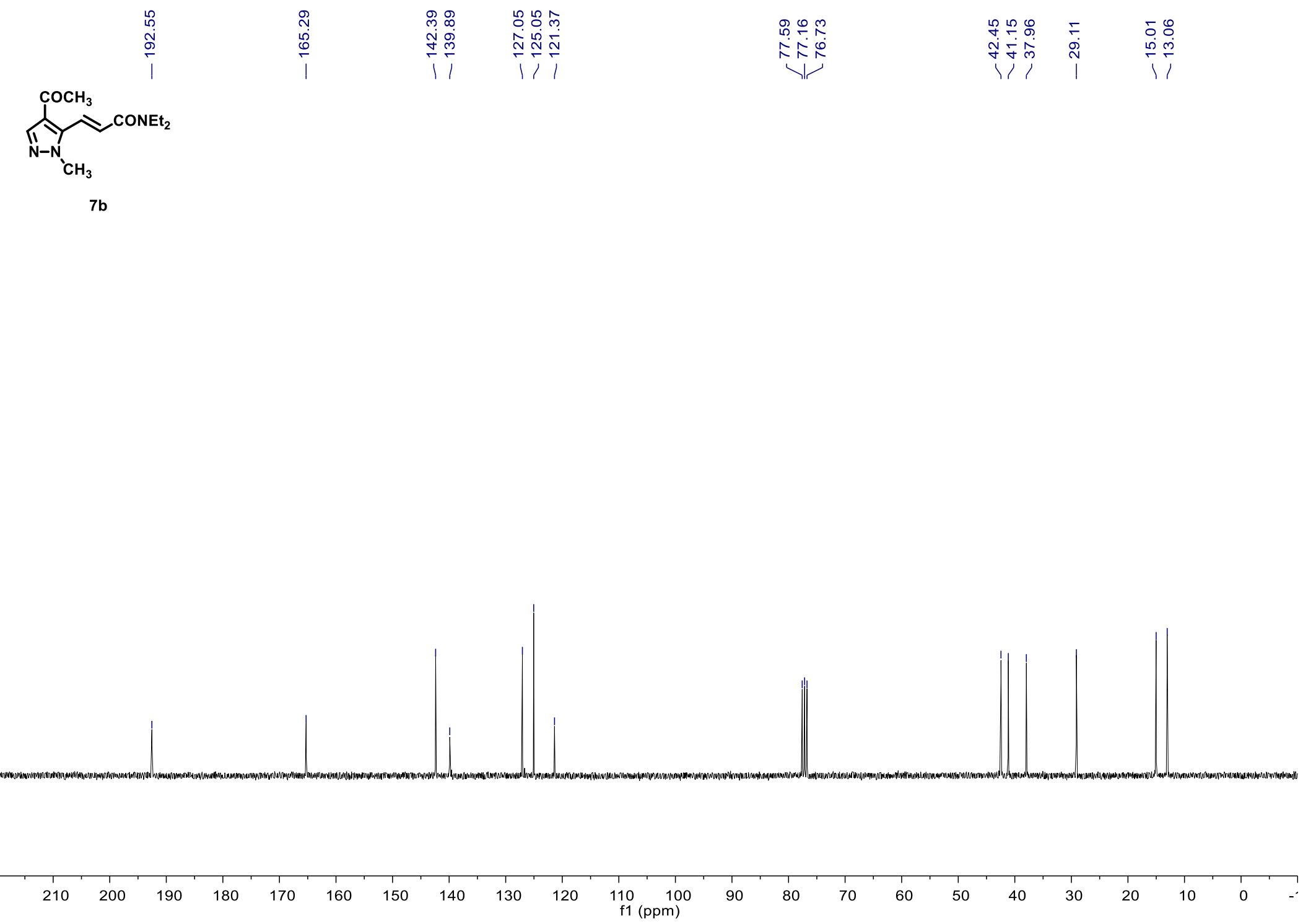

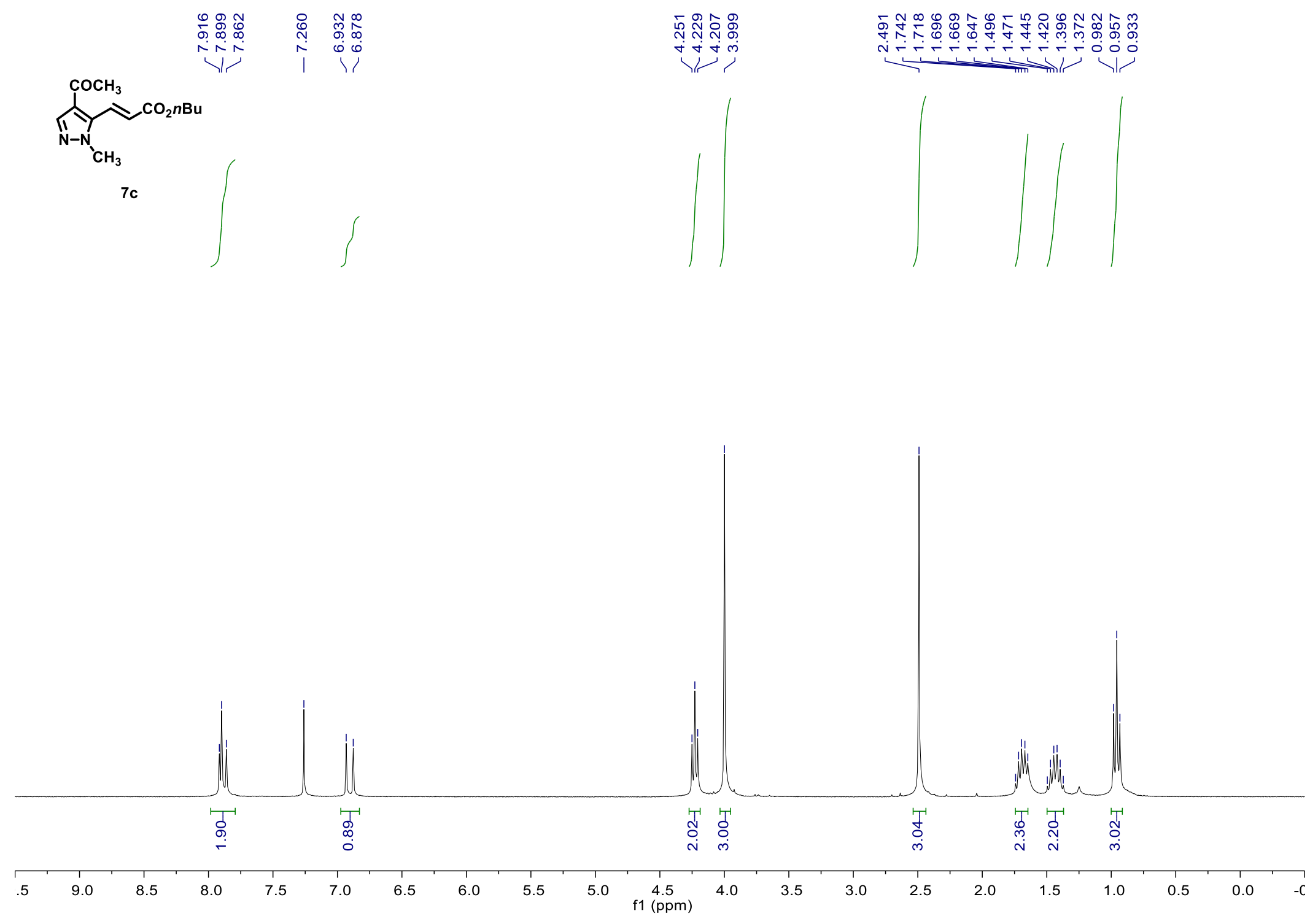

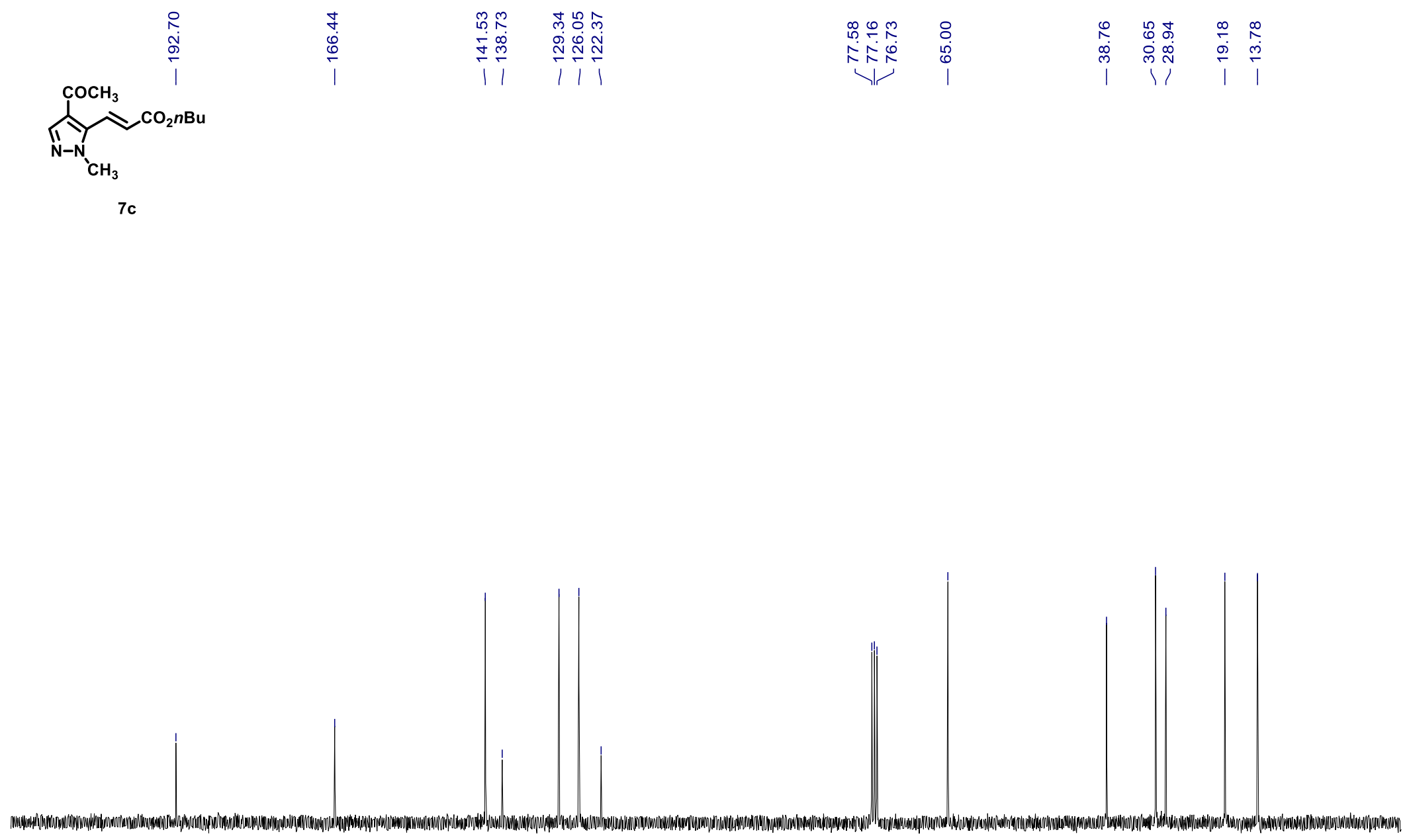

$\begin{array}{llllll}210 & 200 & 190 & 180 & 170 & 160\end{array}$

$150 \quad 140$

$\begin{array}{cccc}130 & 120 & 110 & 100 \\ & f 1(\mathrm{ppm})\end{array}$



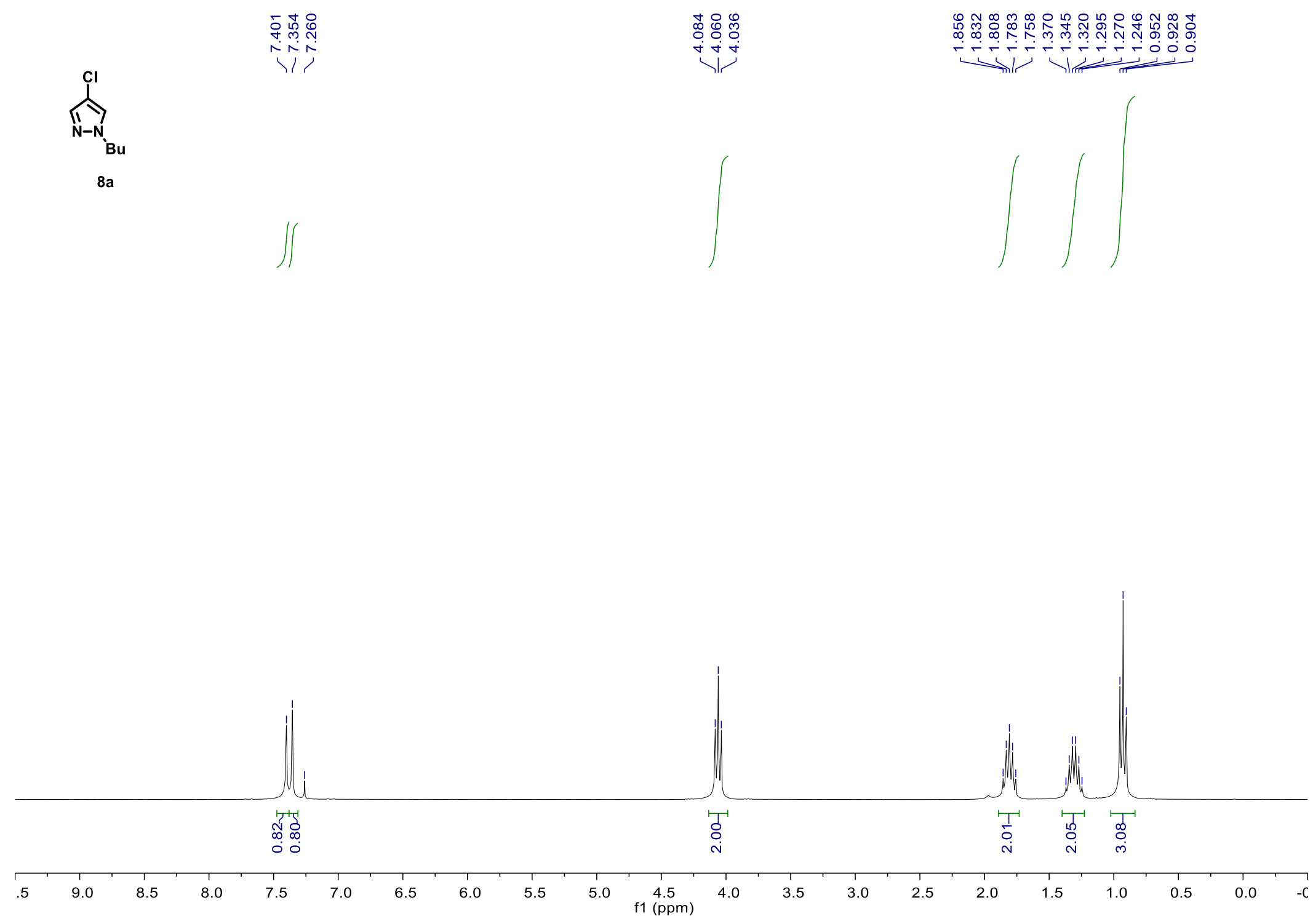
$\overbrace{\mathrm{N}-\mathrm{N}}^{\mathrm{Cl}}$

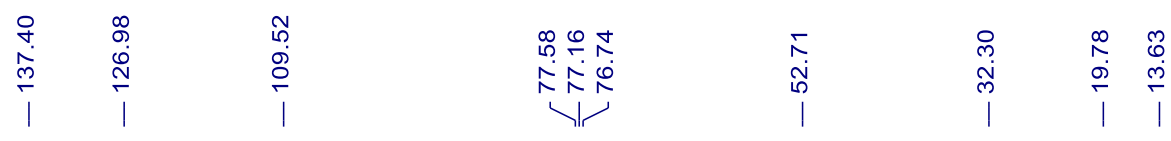

$8 a$

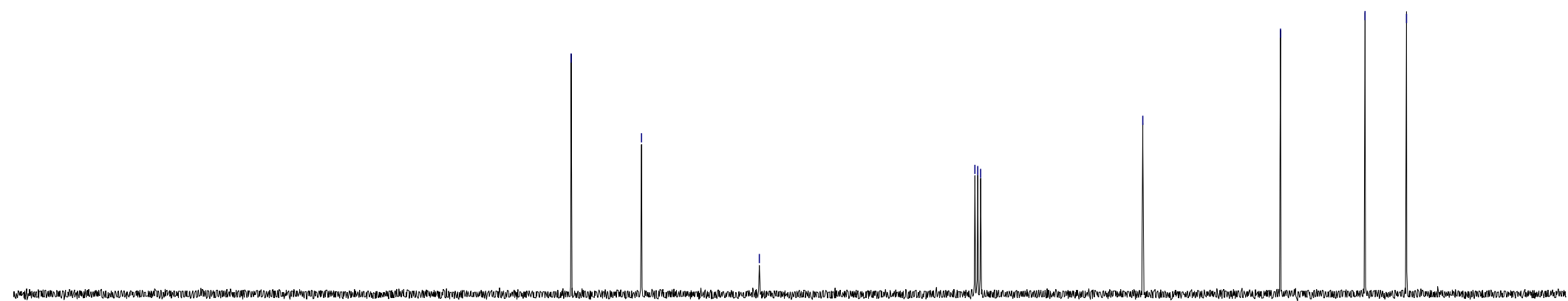

$\begin{array}{lllllllllllllllllllllllllllllll} & 210 & 200 & 190 & 180 & 170 & 160 & 150 & 140 & 130 & 120 & 110 & 100 & 90 & 80 & 70 & 60 & 50 & 40 & 30 & 20 & 10 & 0 & -\end{array}$ 

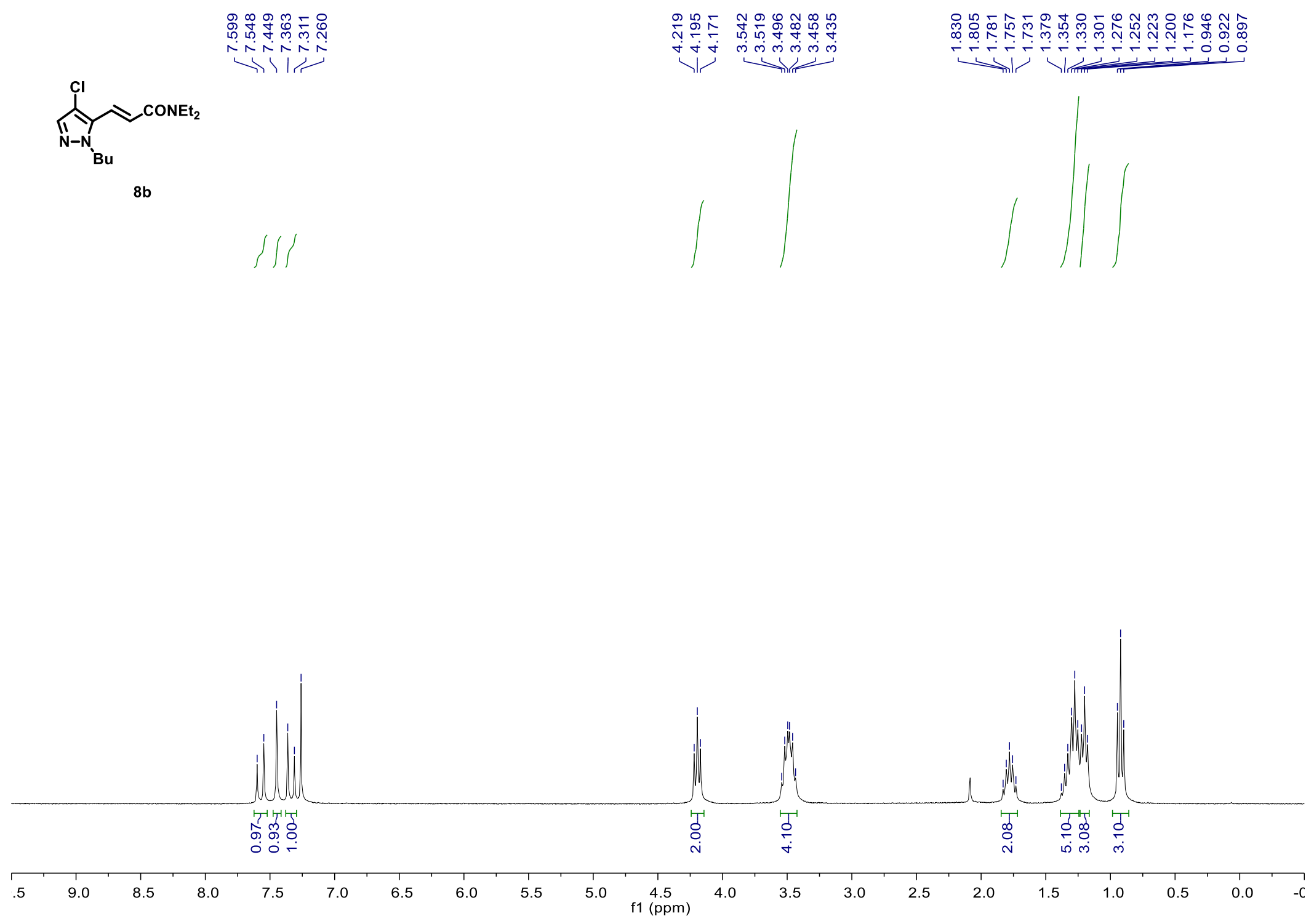


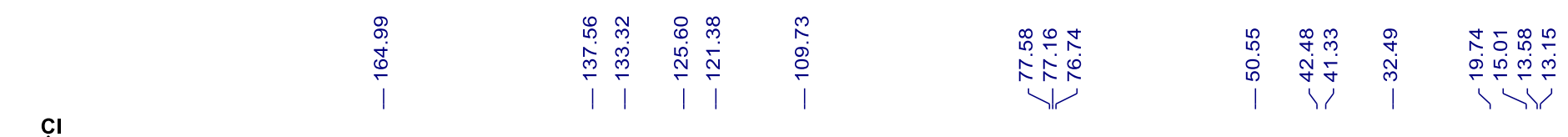

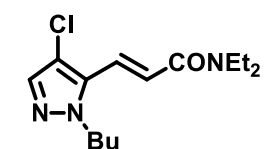

$8 b$

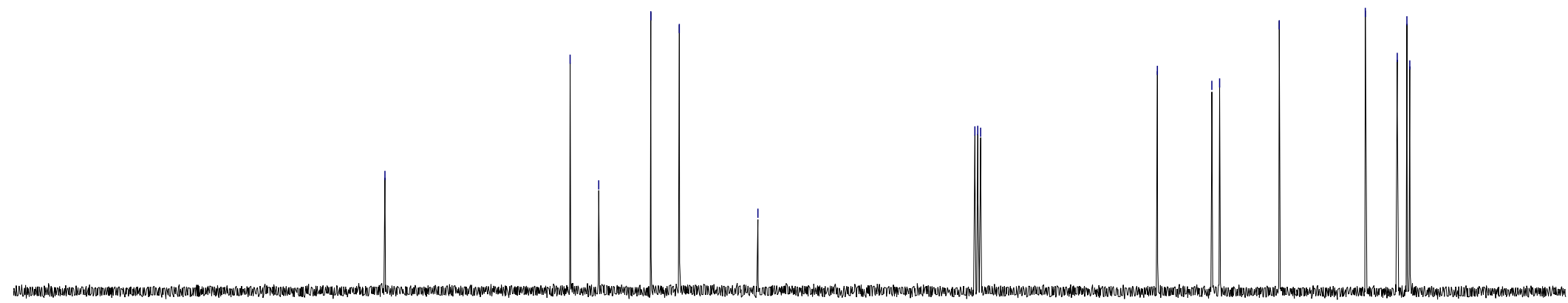

$\begin{array}{lllllllllllllllllllllllll}210 & 200 & 190 & 180 & 170 & 160 & 150 & 140 & 130 & 120 & 110 & 100 & 90 & 80 & 70 & 60 & 50 & 40 & 30 & 20 & 10 & 0 & -\end{array}$ 


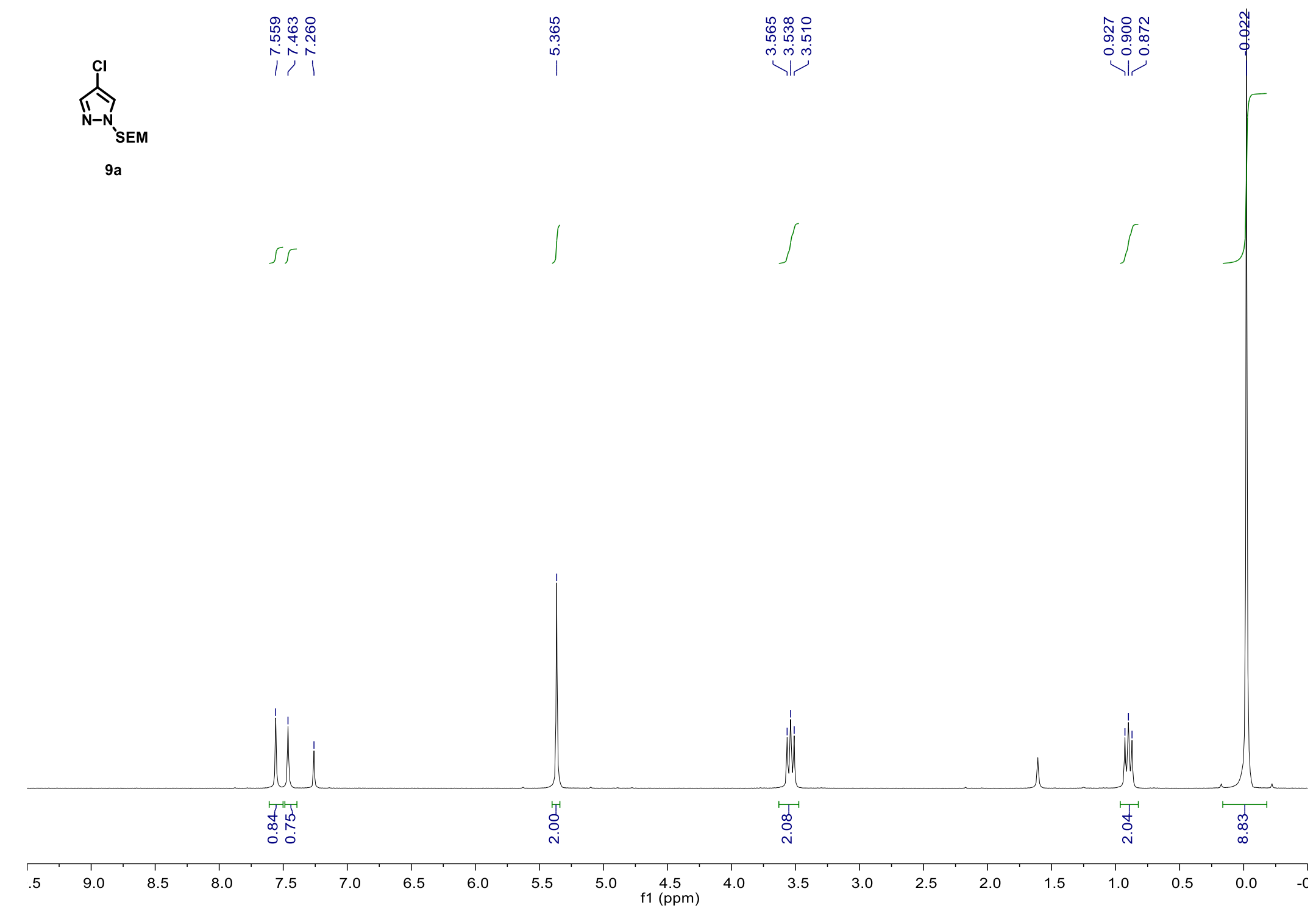




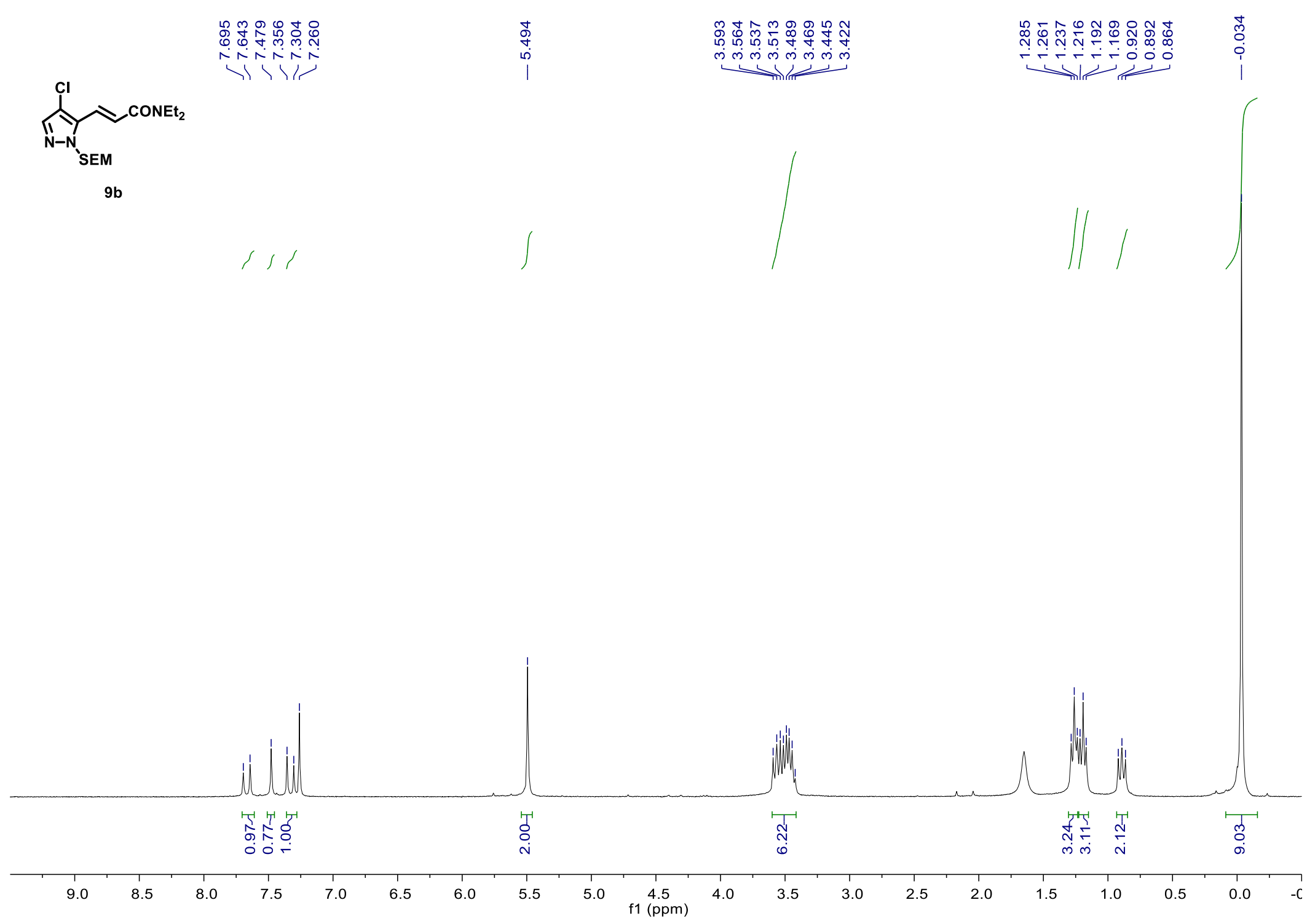



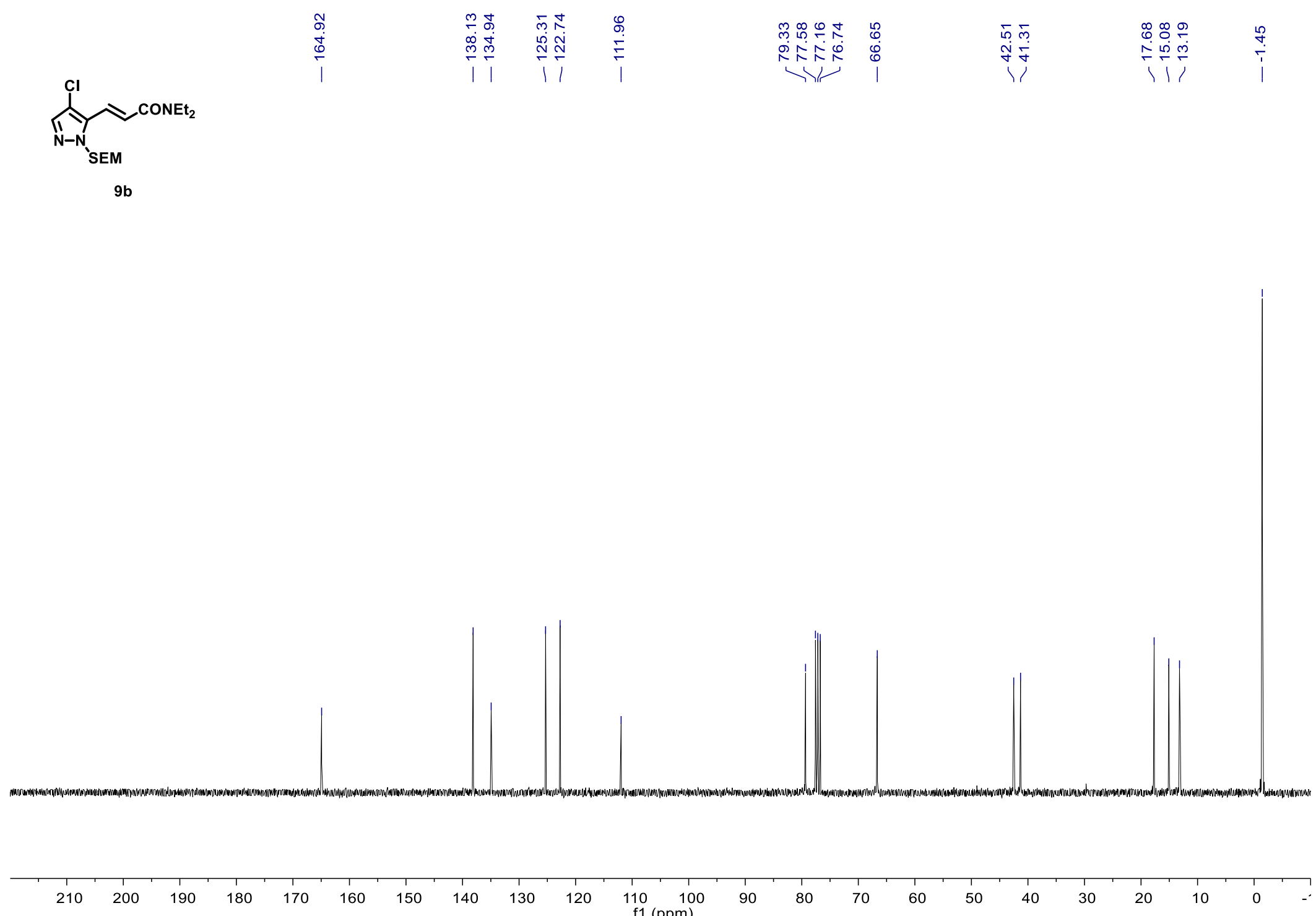
总素送

:

$\mathbb{N}_{\mathrm{N}-\mathrm{CH}_{3}}^{\mathrm{Cl}}$

10a

II

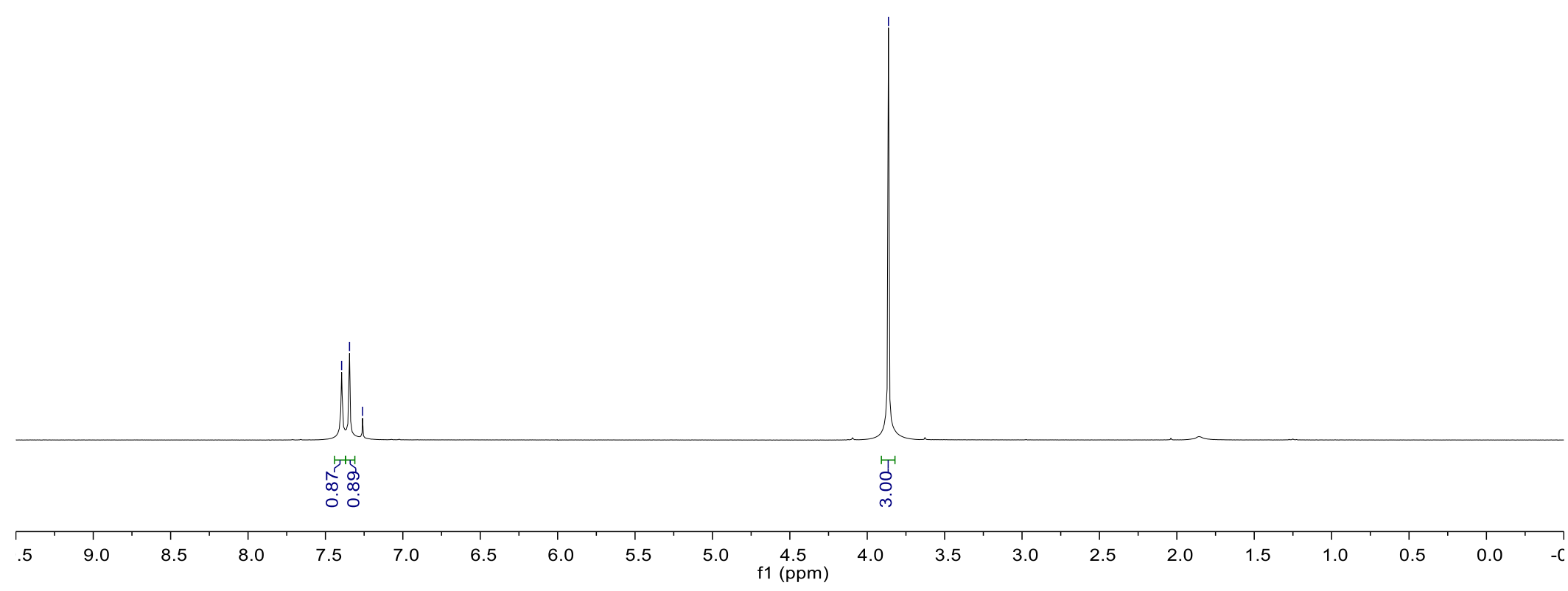



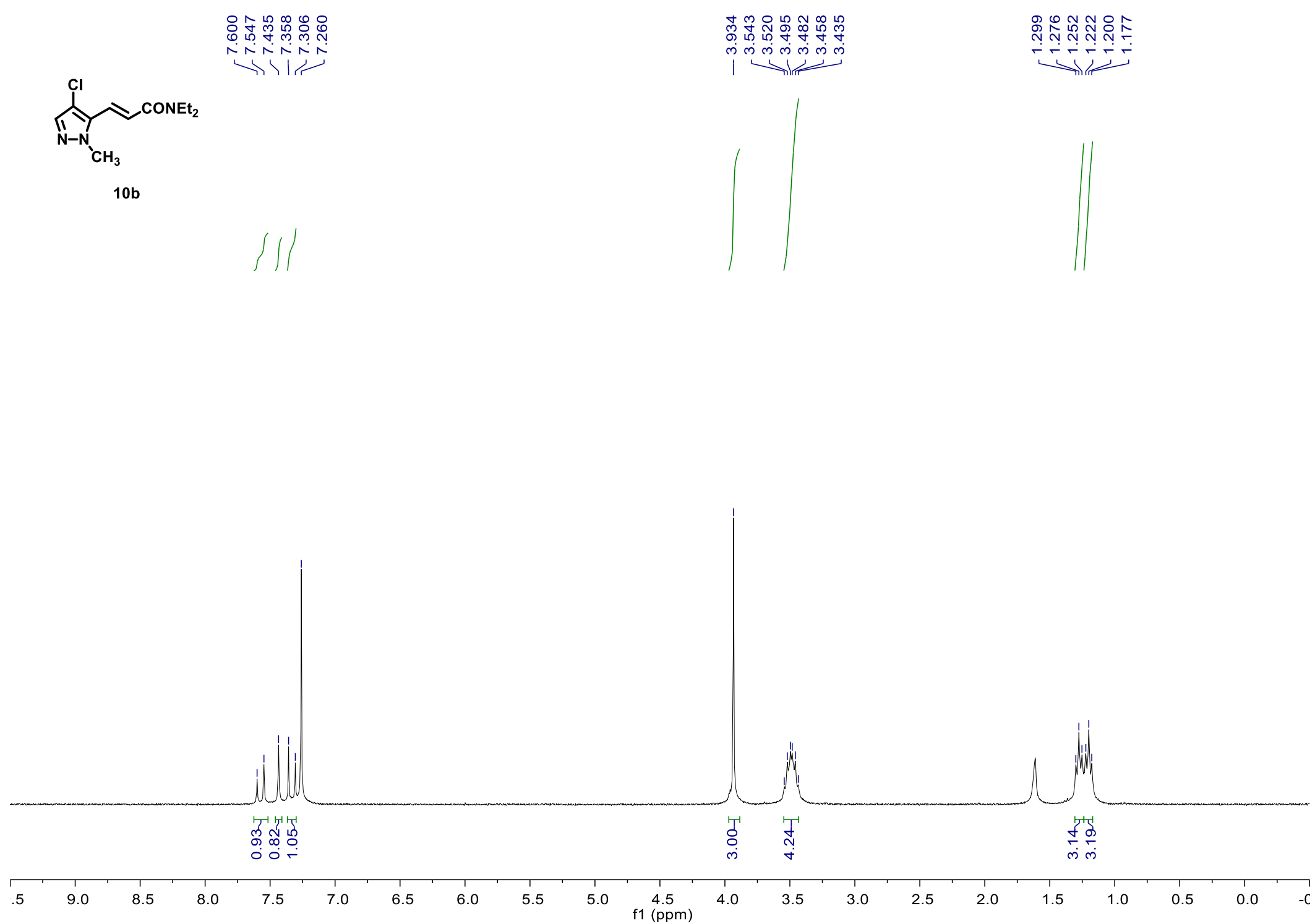


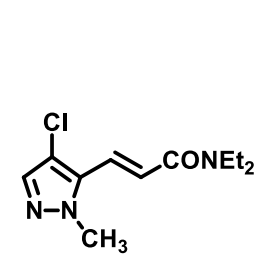

每

$10 \mathrm{~b}$

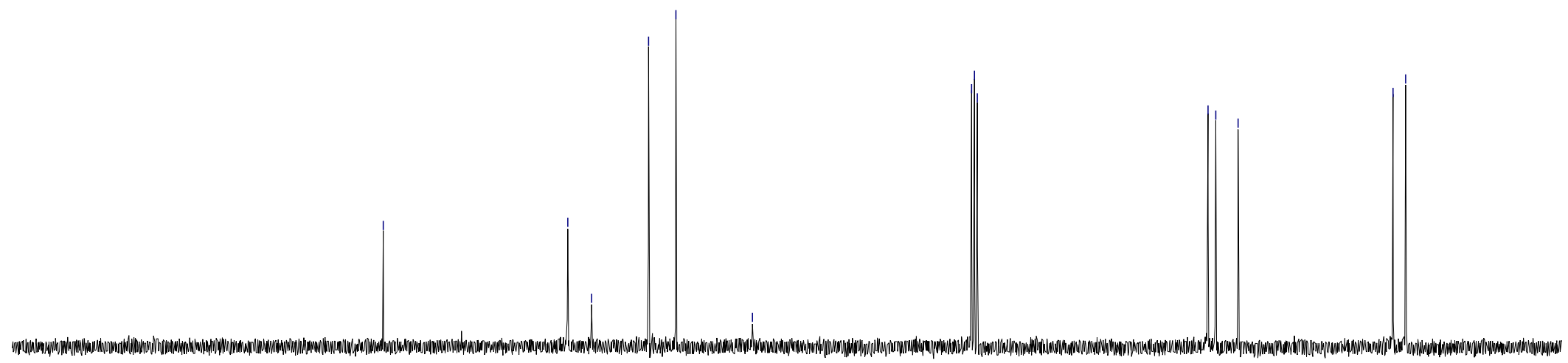

$\begin{array}{lllll}1 & 1 & 1 & & \\ 210 & 200 & 190 & 180 & 170\end{array}$ 

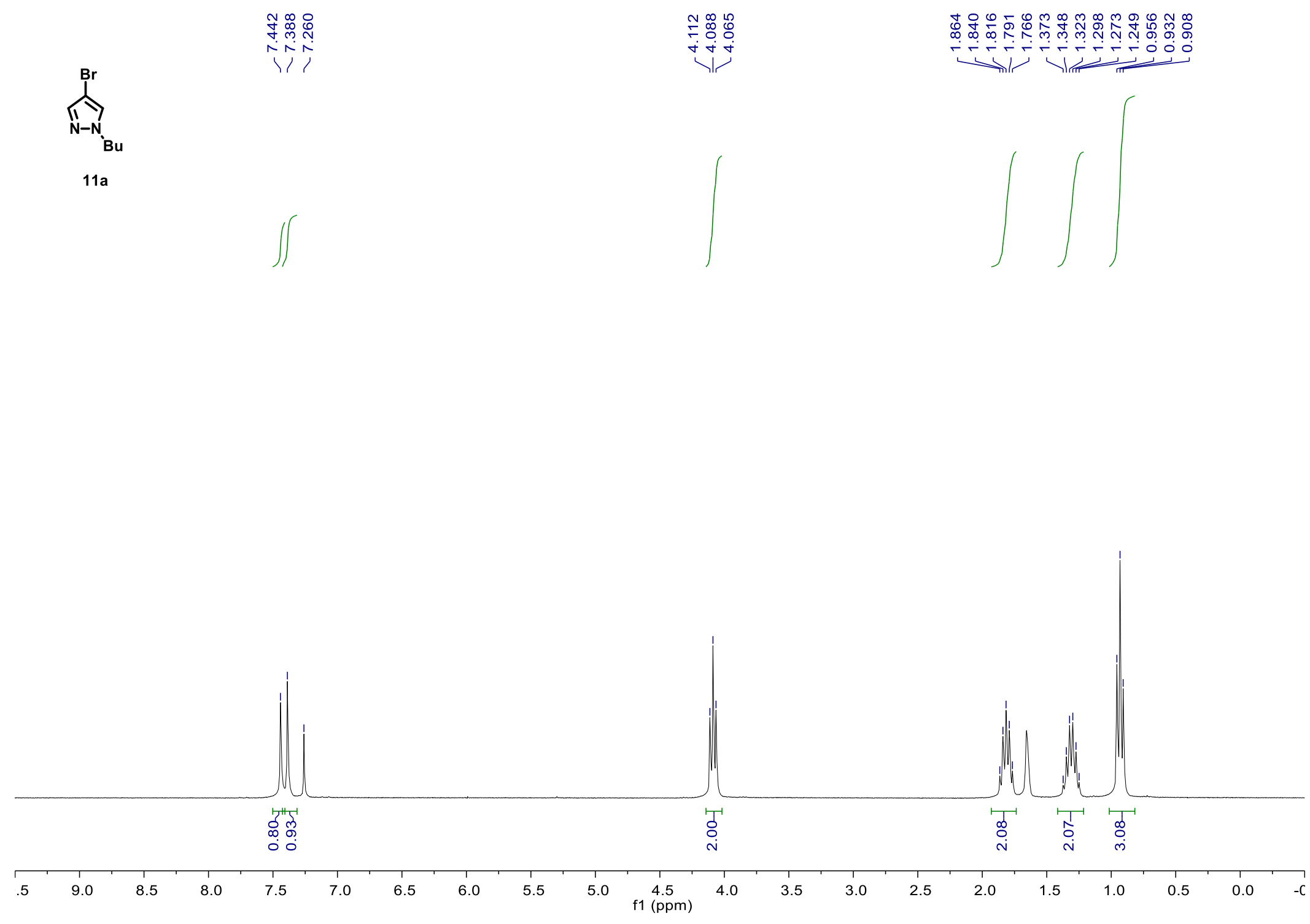


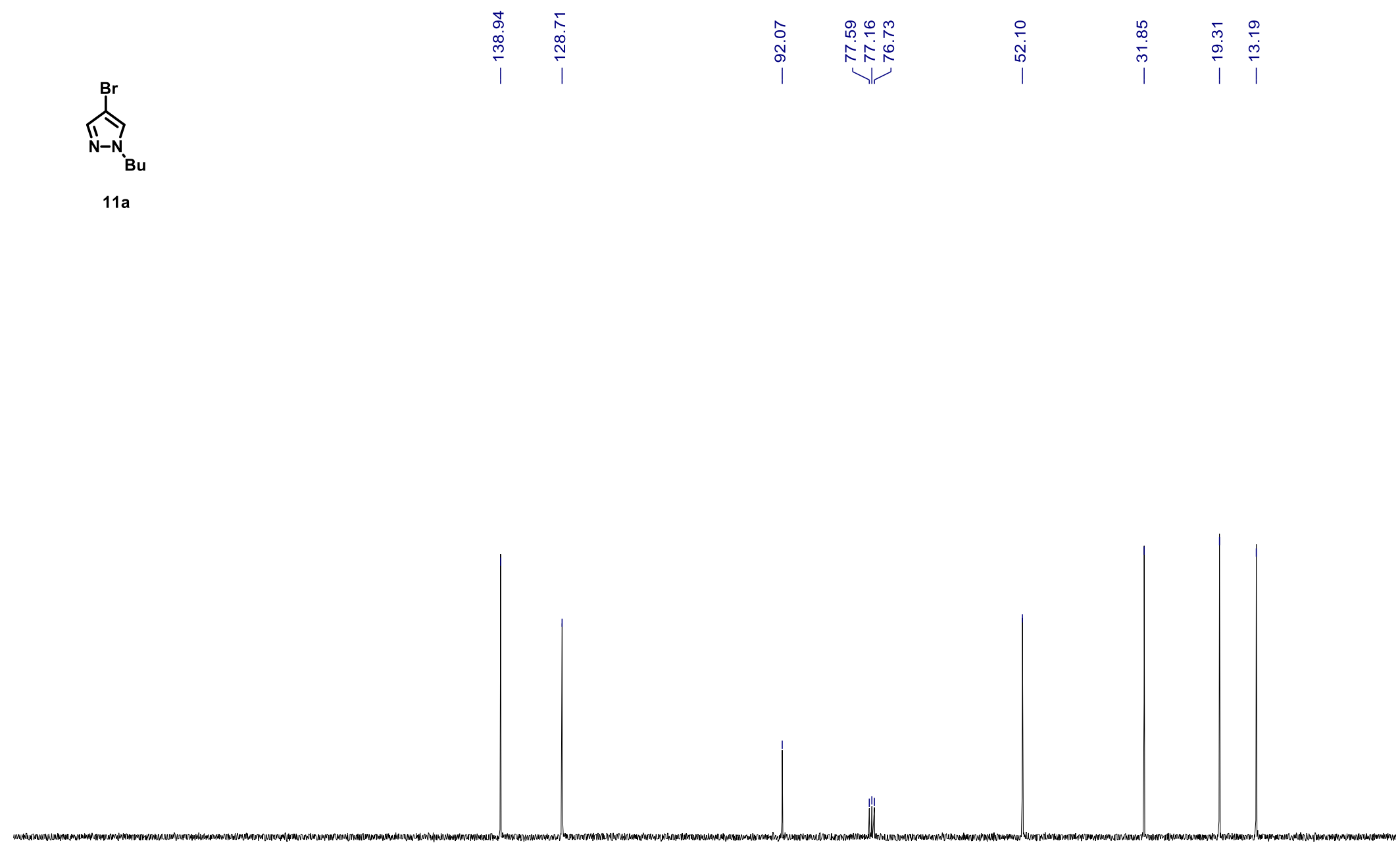

$\begin{array}{lllllllllllllllllllllllllllll} & 210 & 200 & 190 & 180 & 170 & 160 & 150 & 140 & 130 & 120 & 110 & 100 & 90 & 80 & 70 & 60 & 50 & 40 & 30 & 20 & 10 & 0 & 0\end{array}$ 


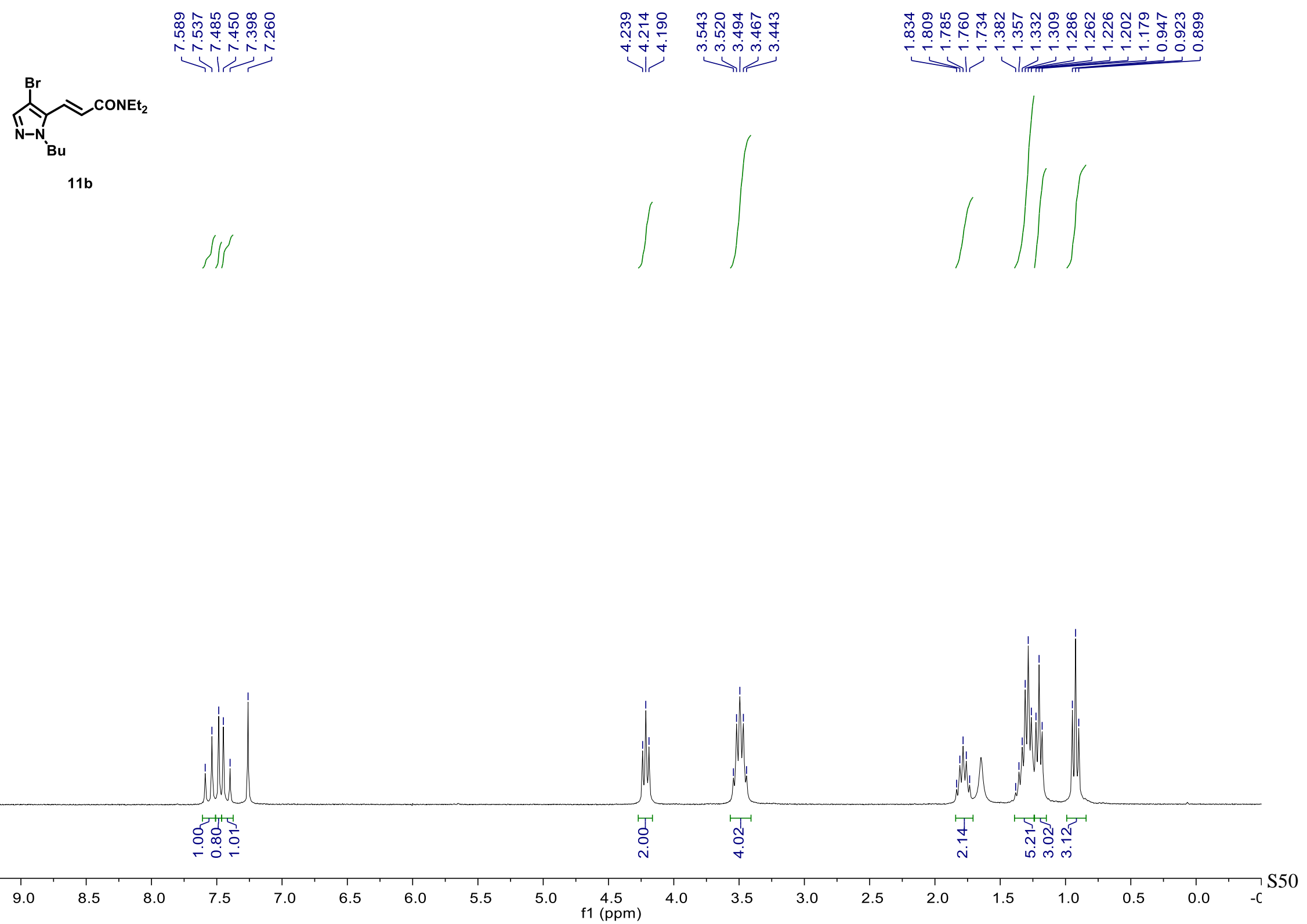



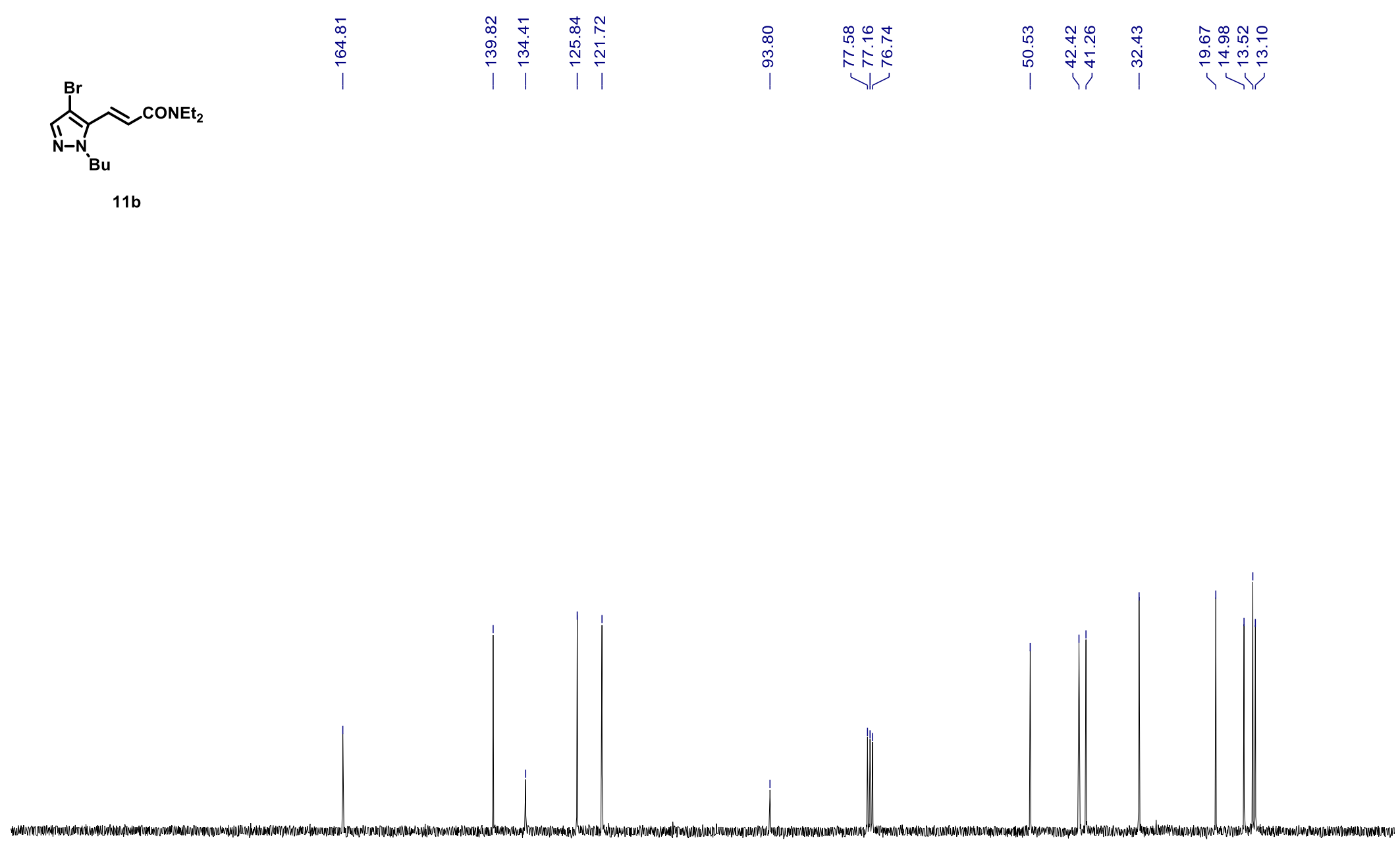

$\begin{array}{lllllllllllllllllllllllll}210 & 200 & 190 & 180 & 170 & 160 & 150 & 140 & 130 & 120 & 110 & 100 & 90 & 80 & 70 & 60 & 50 & 40 & 30 & 20 & 10 & 0 & -\end{array}$ 


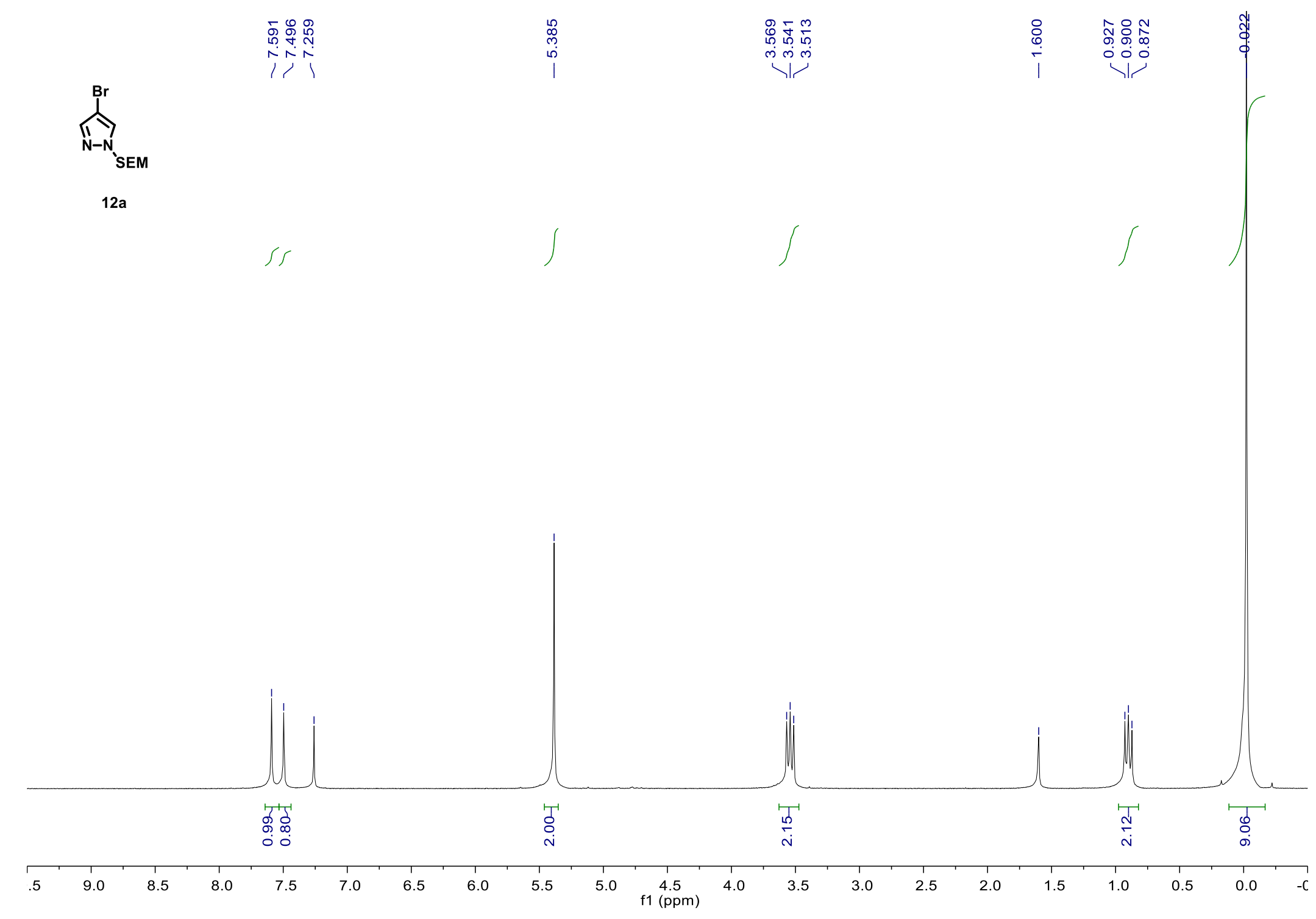




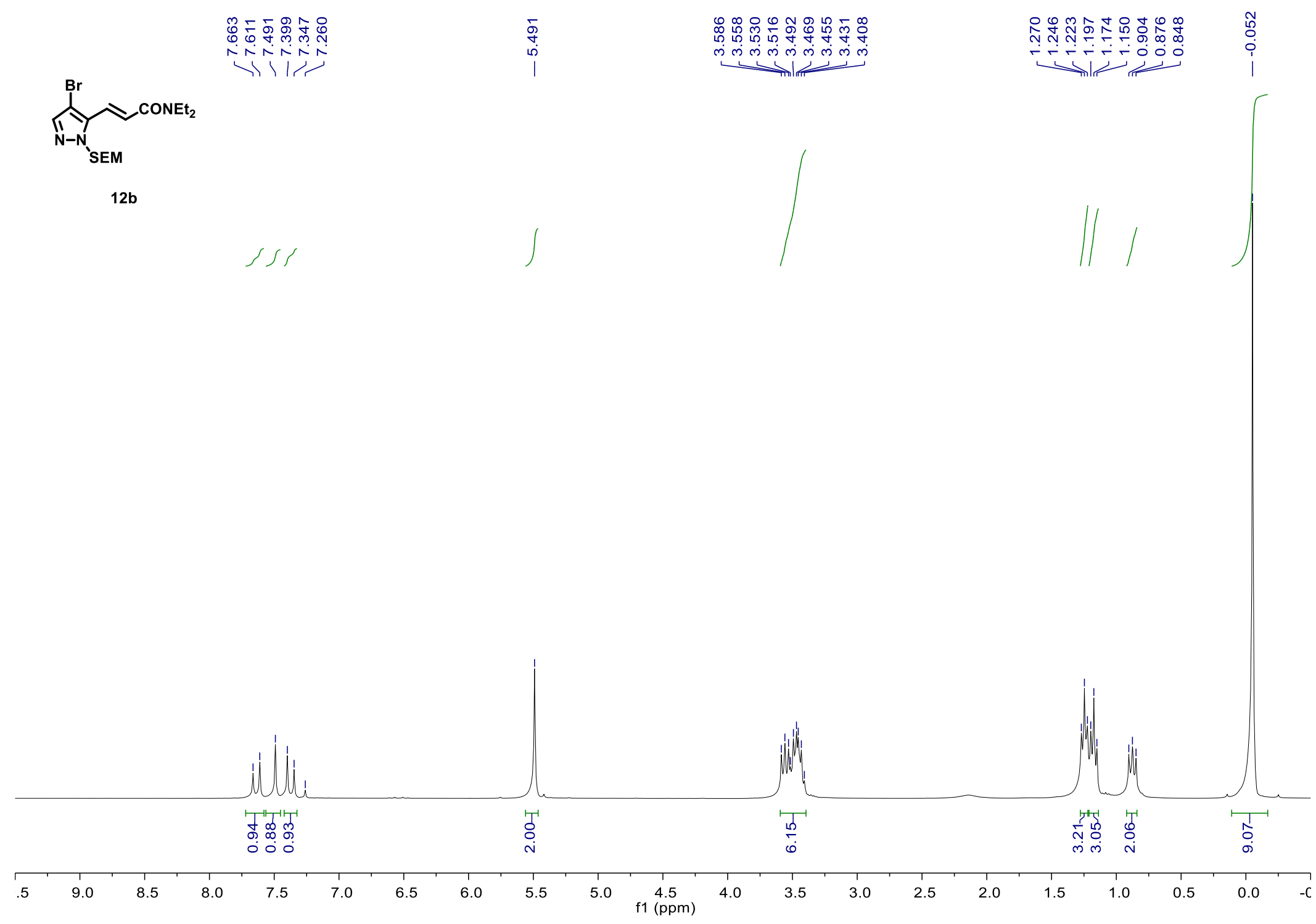



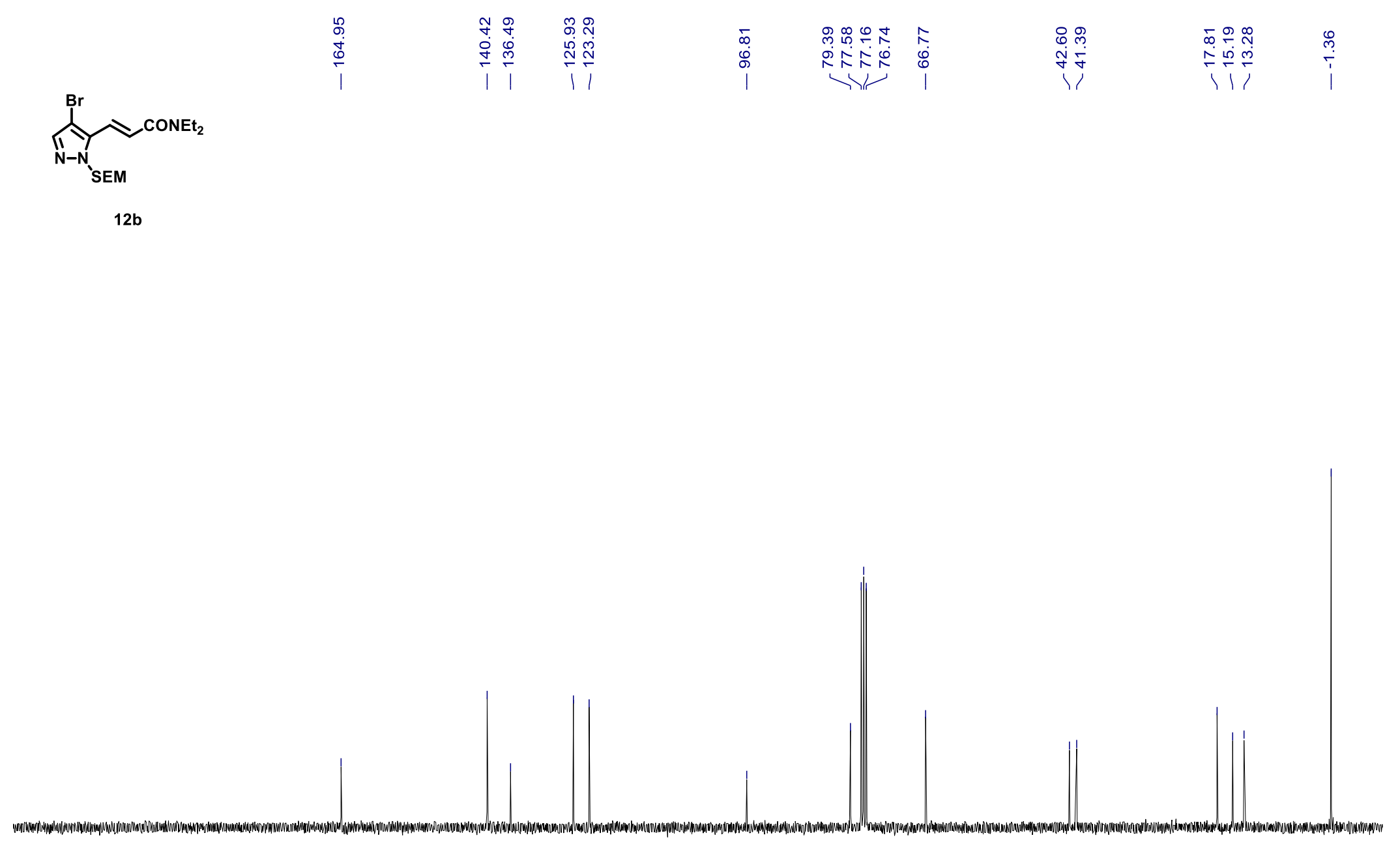

$\begin{array}{llllllllllllllllllllllllllll}210 & 200 & 190 & 180 & 170 & 160 & 150 & 140 & 130 & 120 & 110 & 100 & 90 & 80 & 70 & 60 & 50 & 40 & 30 & 20 & 10 & 0 & -\end{array}$ 


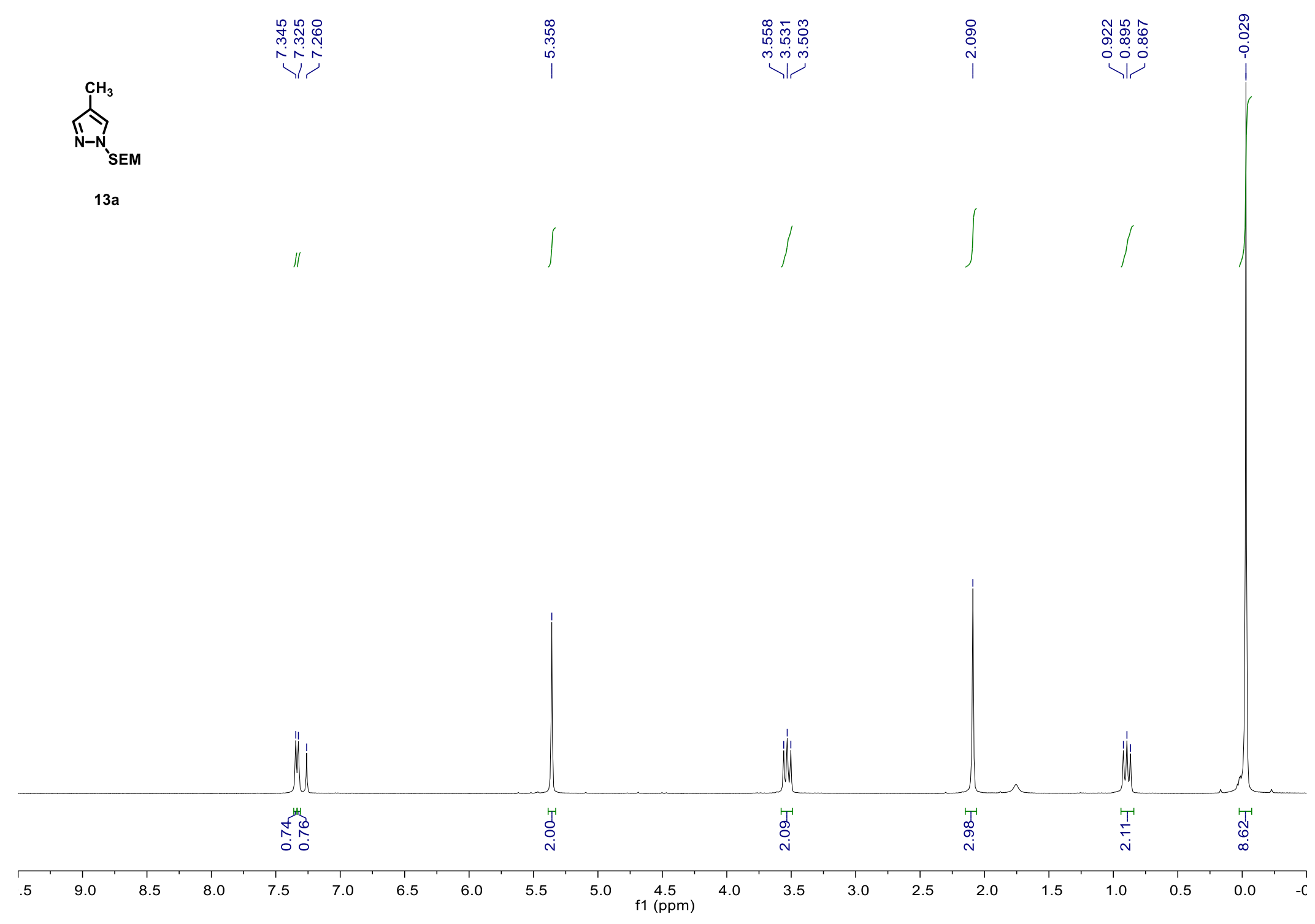




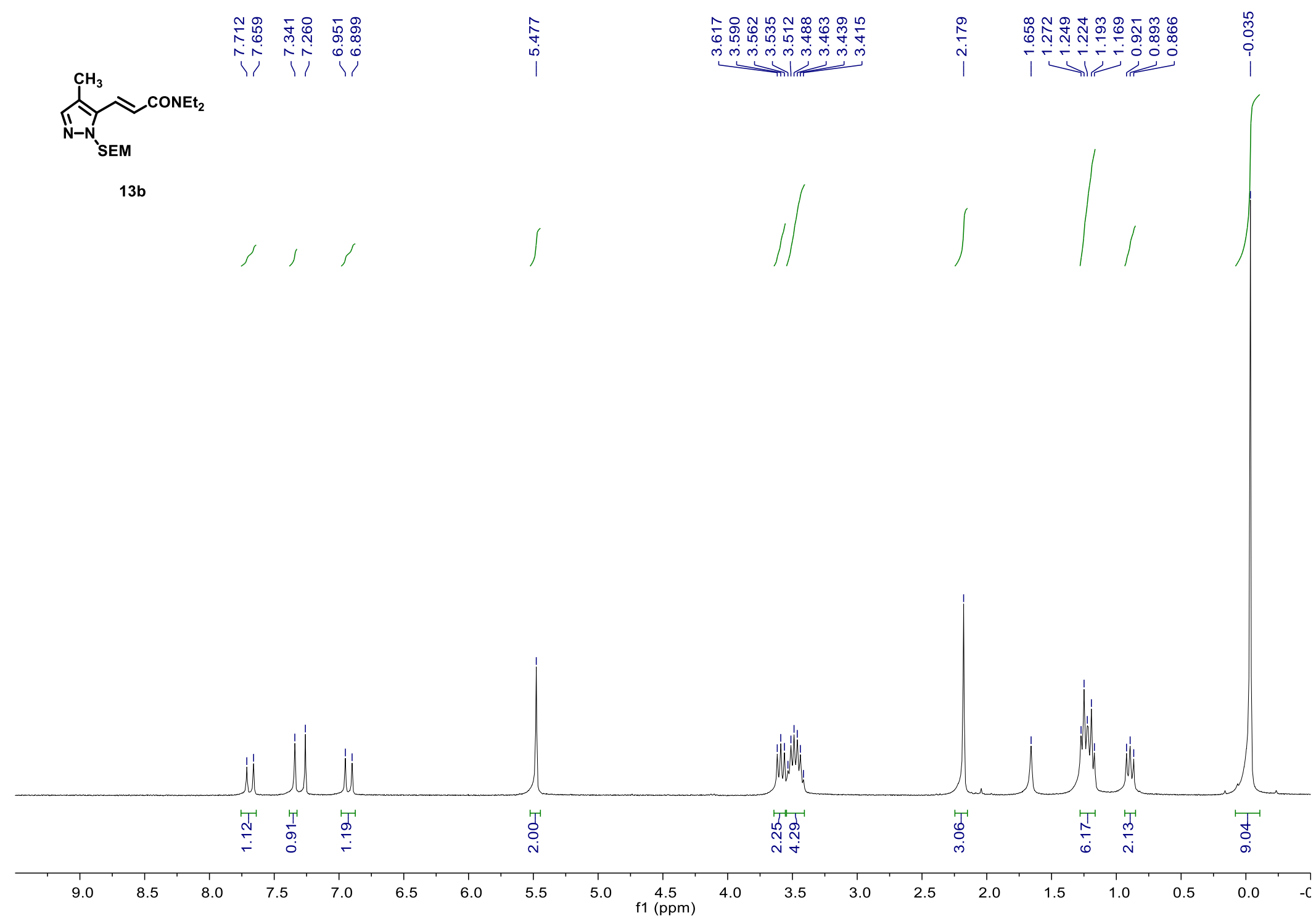



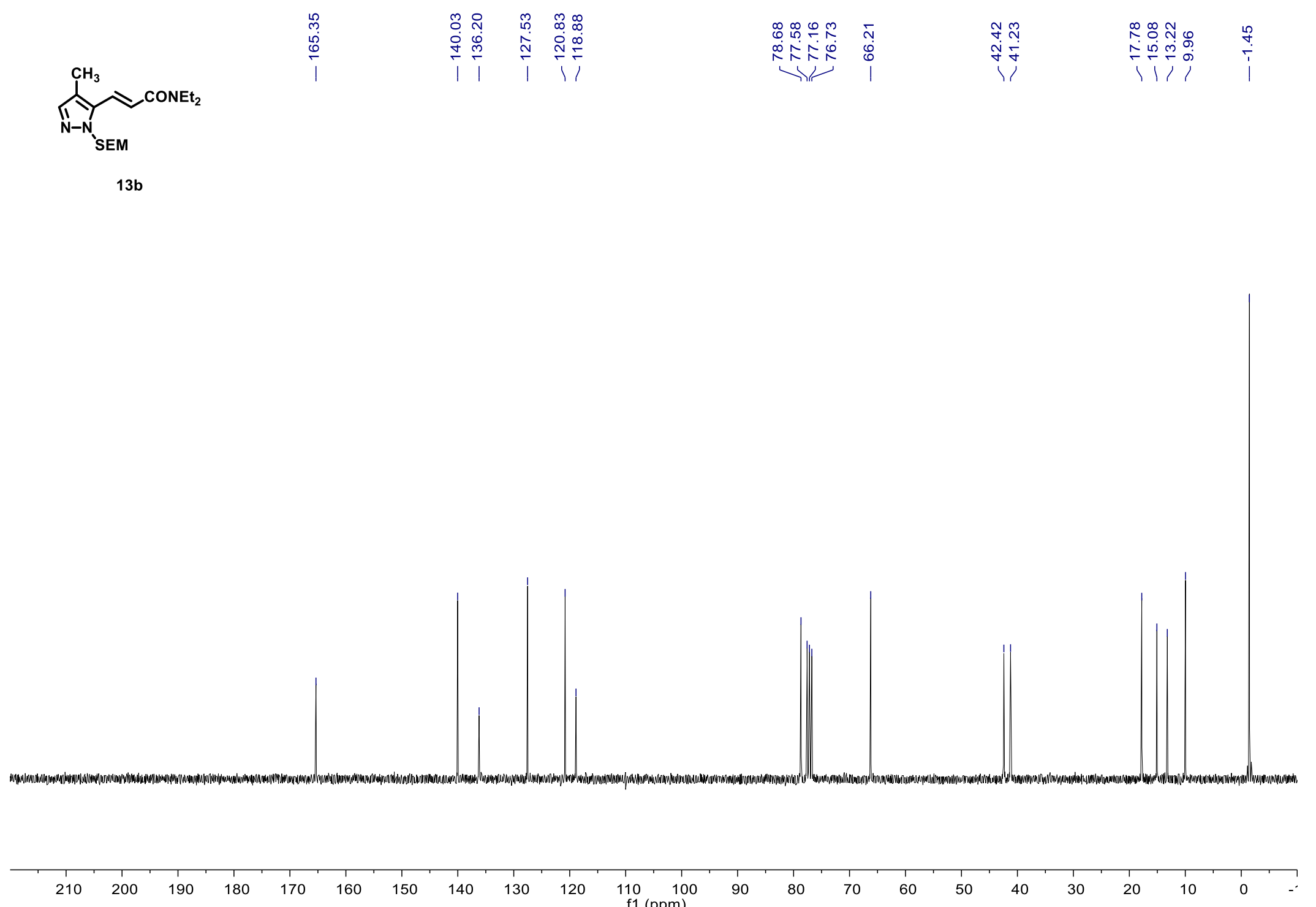

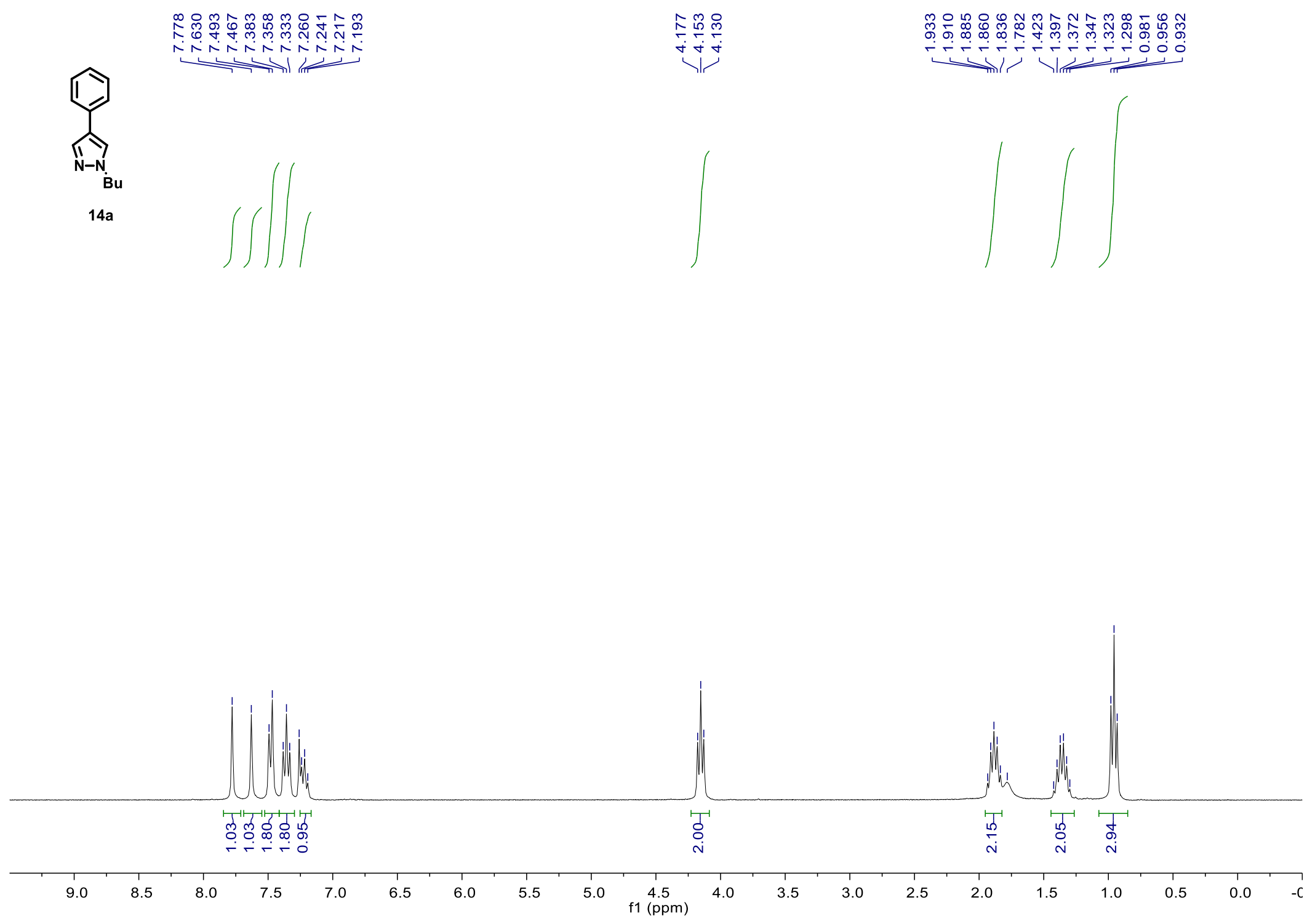

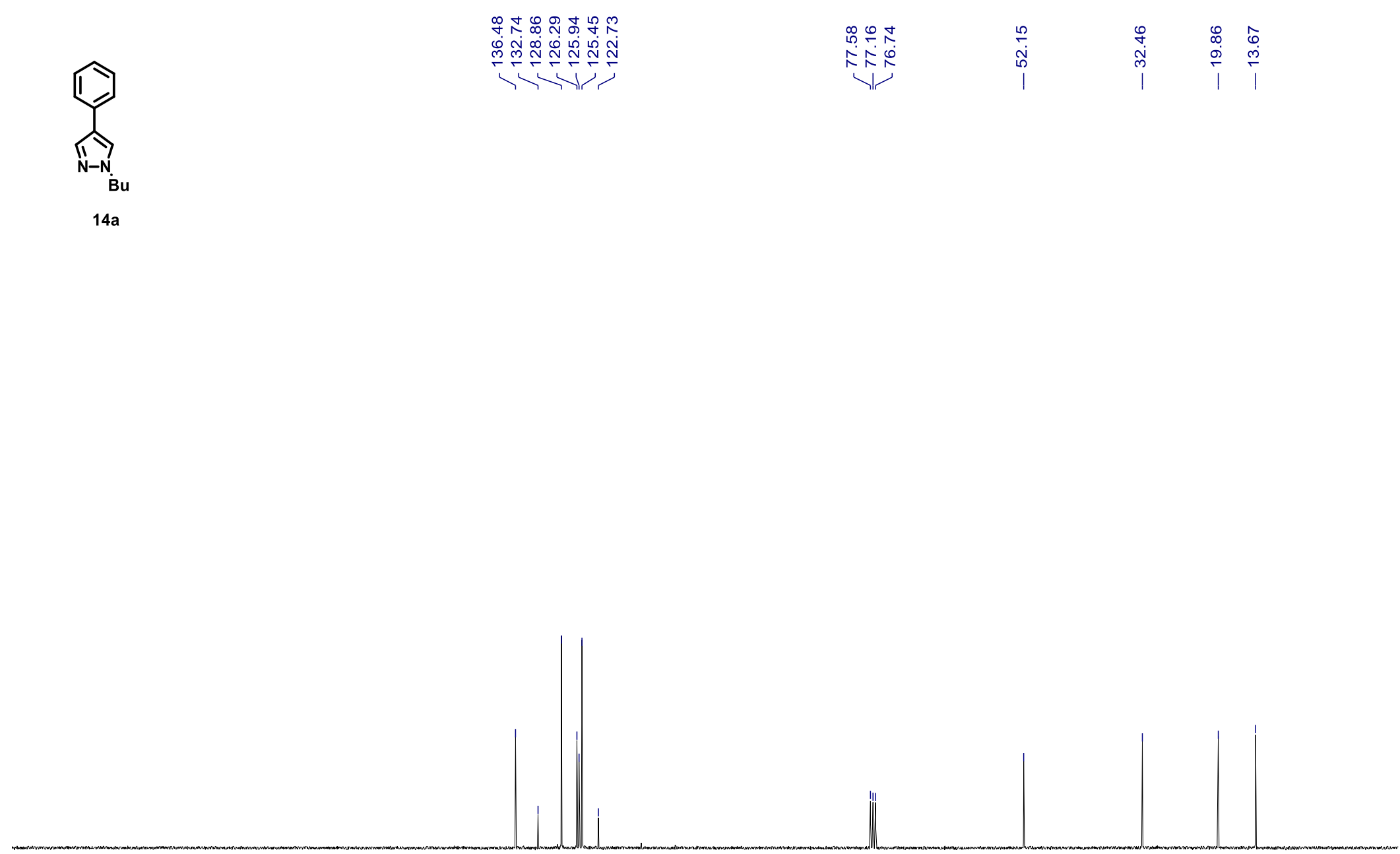

$\begin{array}{lllllllllllllllllllllllll} & 210 & 200 & 190 & 180 & 170 & 160 & 150 & 140 & 130 & 120 & 110 & 100 & 90 & 80 & 70 & 60 & 50 & 40 & 30 & 20 & 10 & 0 & -\end{array}$ 

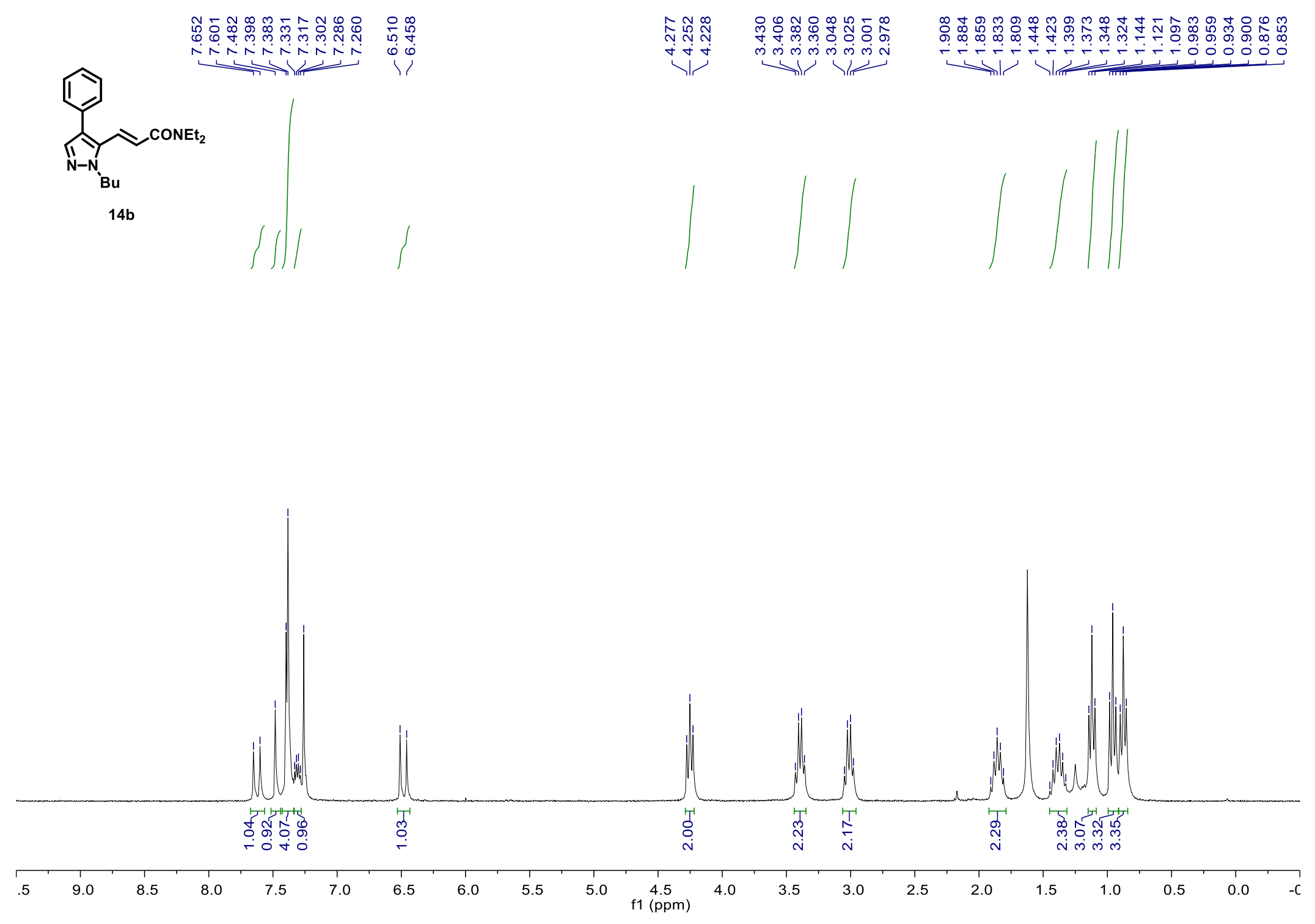

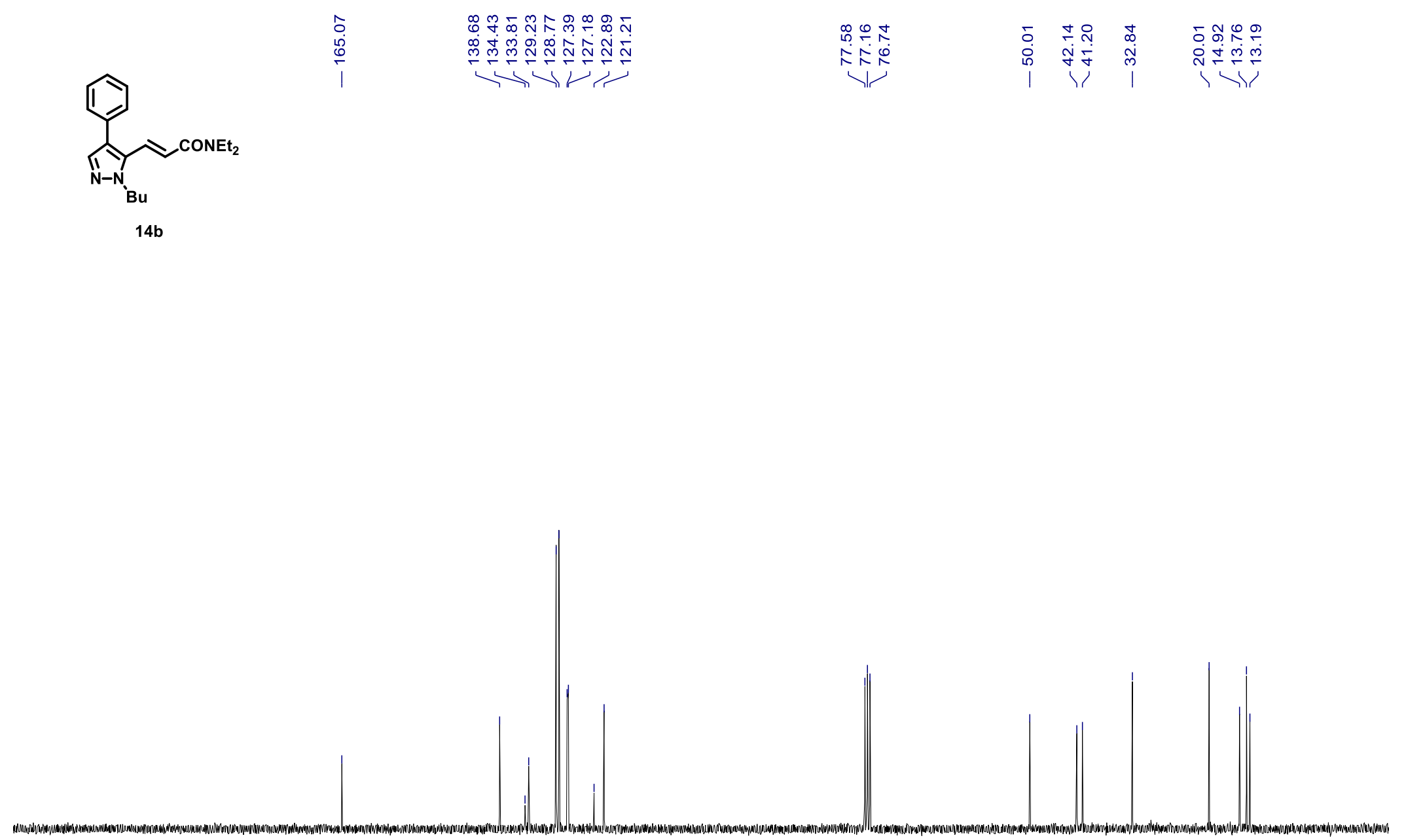

$\begin{array}{llllllllllllllllllllllllll}210 & 200 & 190 & 180 & 170 & 160 & 150 & 140 & 130 & 120 & 110 & 100 & 90 & 80 & 70 & 60 & 50 & 40 & 30 & 20 & 10 & 0 & -\end{array}$ 


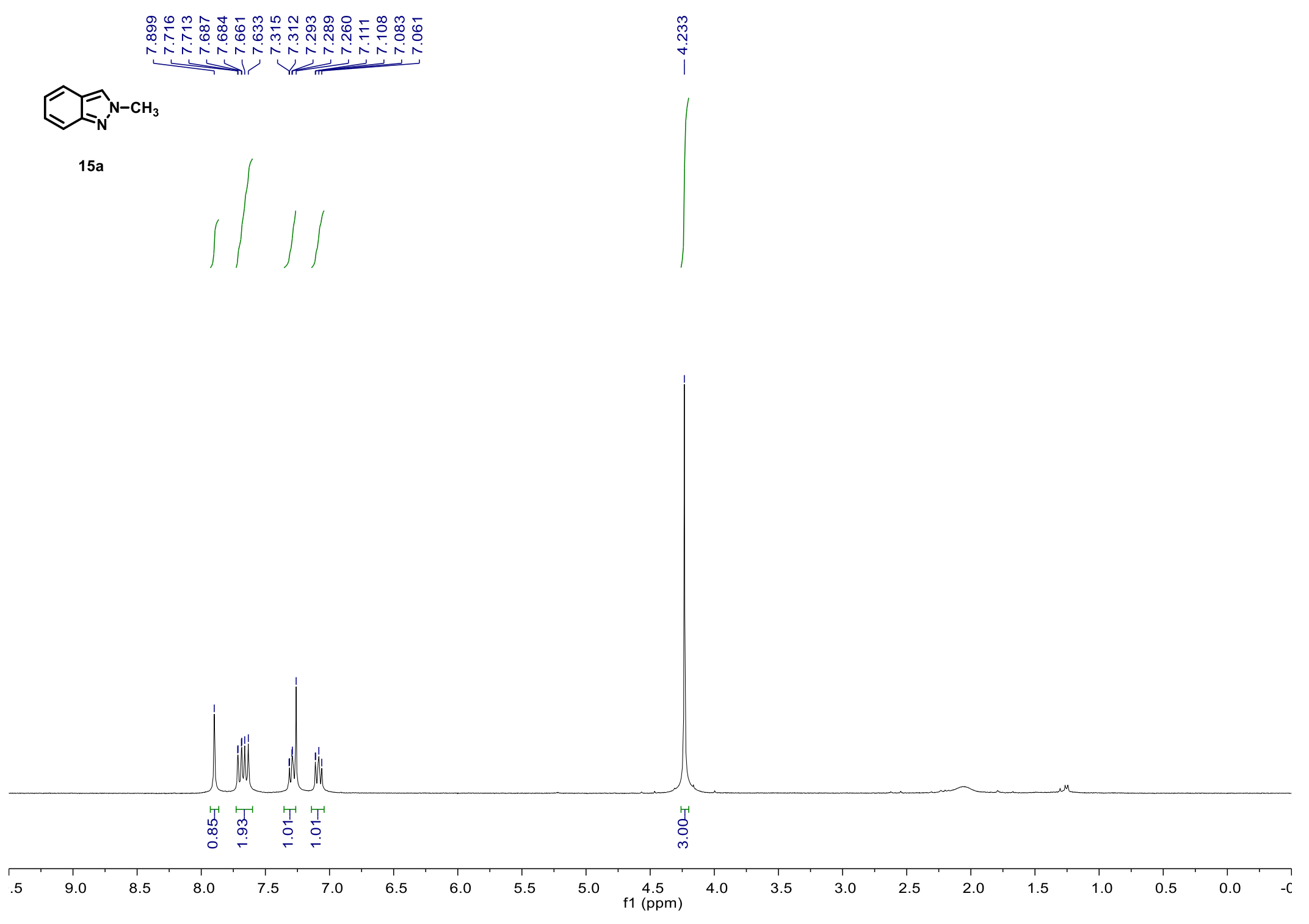



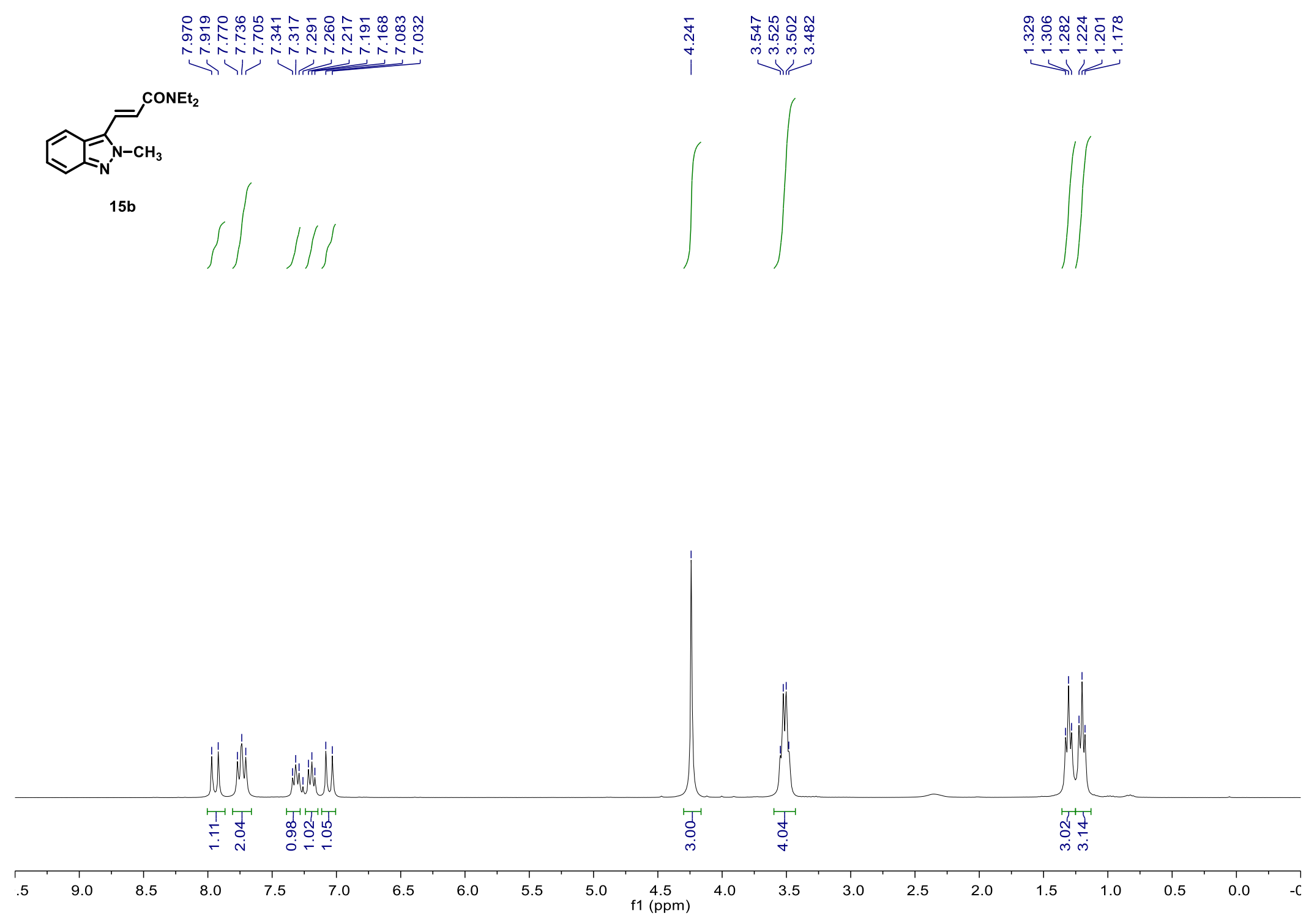


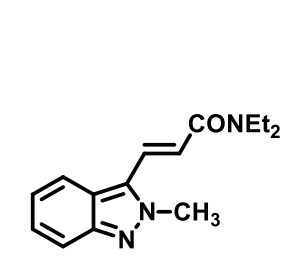

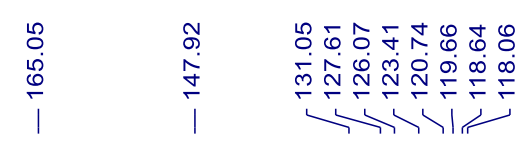

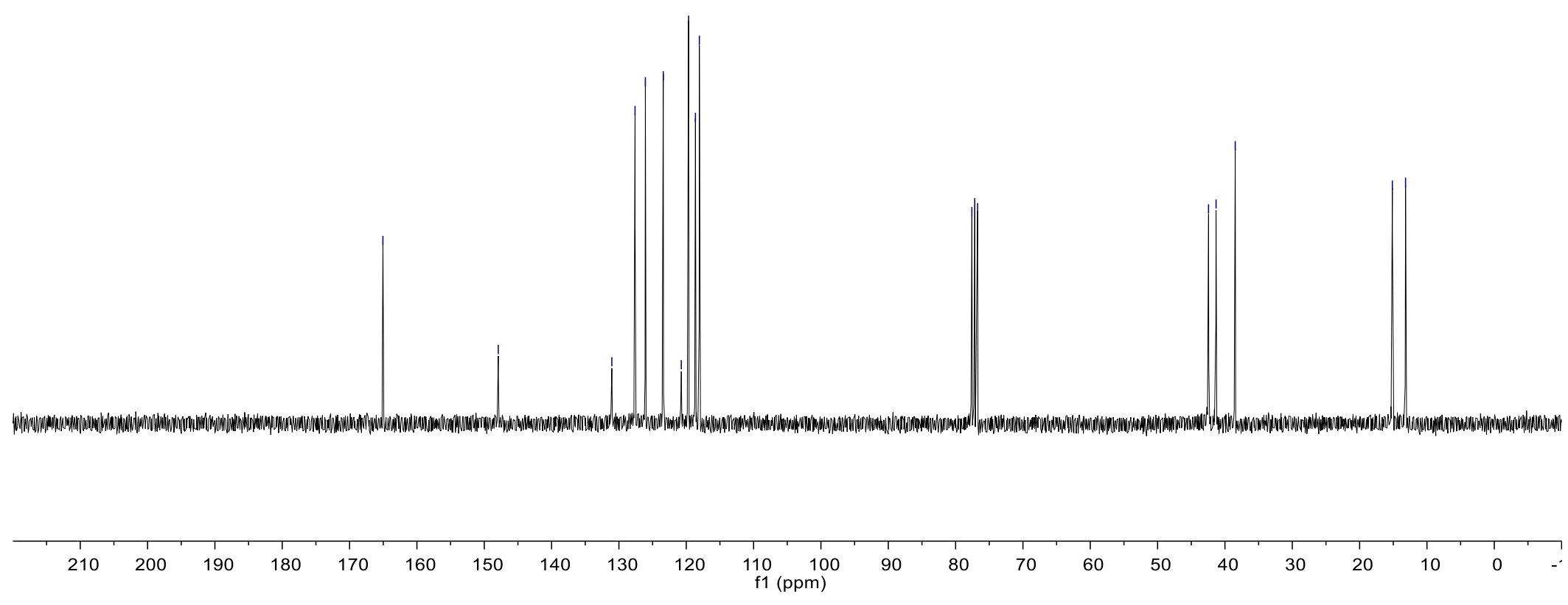




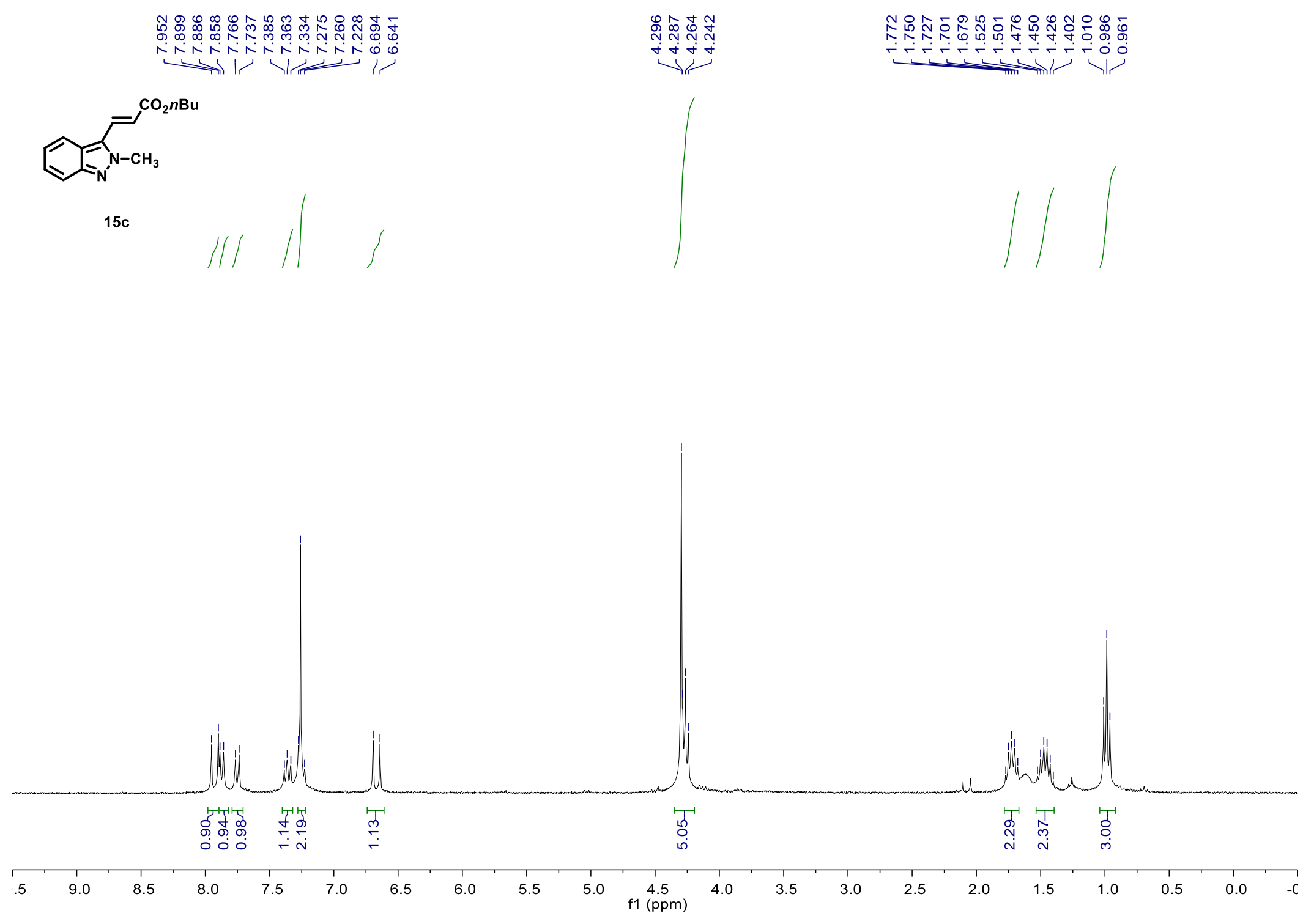




$$
\text { 然 }
$$

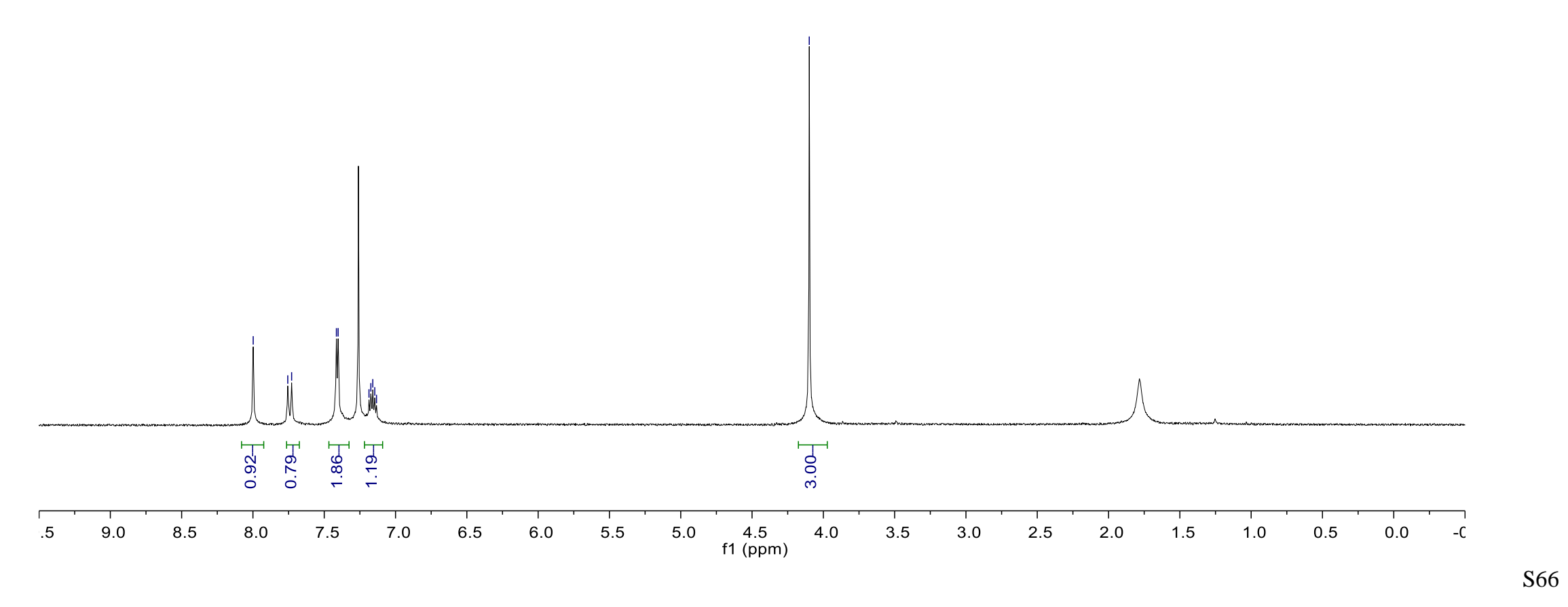



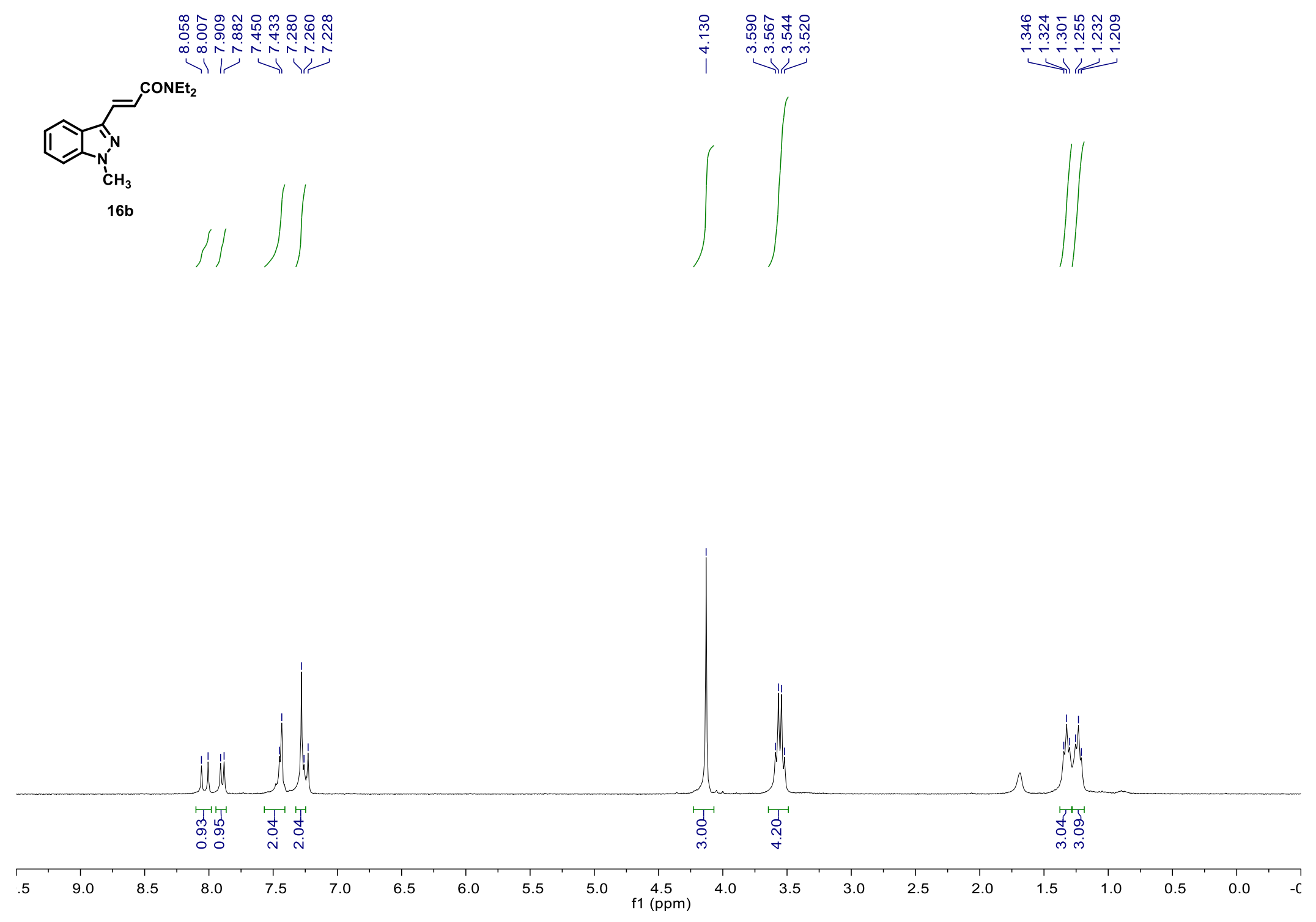

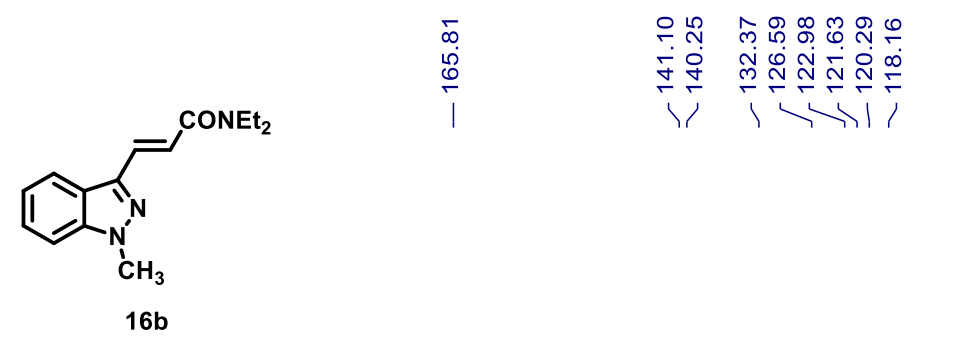

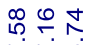

사요

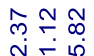

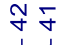

$\stackrel{\infty}{N}$

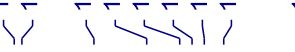

$\downarrow$

I)

$\stackrel{10}{1}$

$16 \mathrm{~b}$

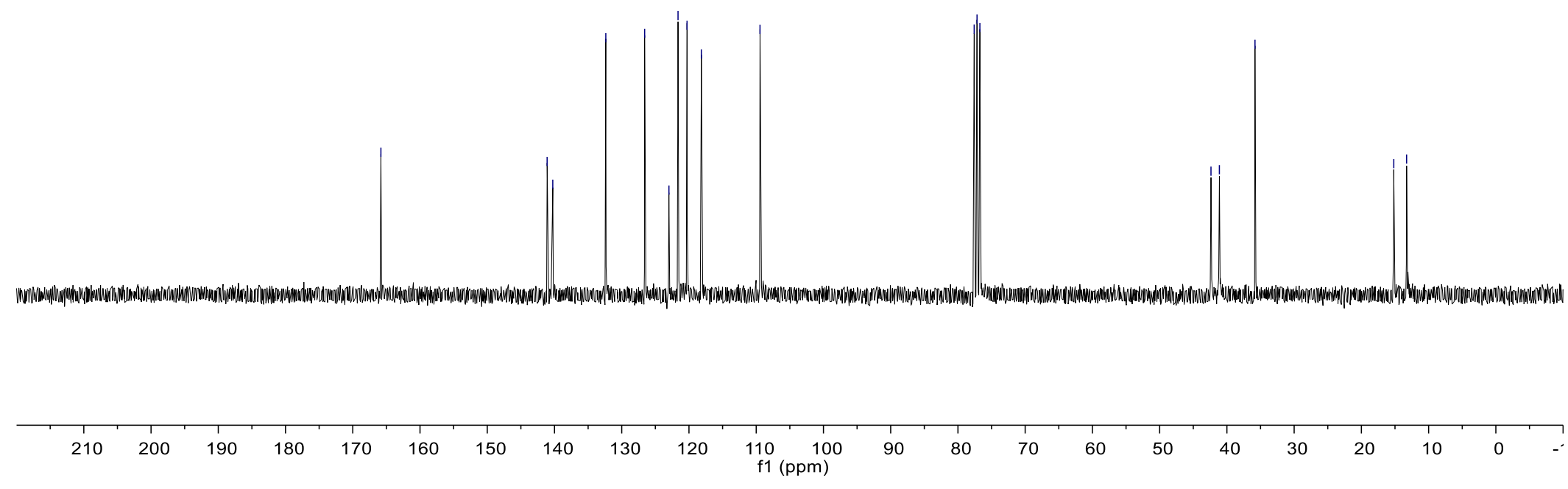



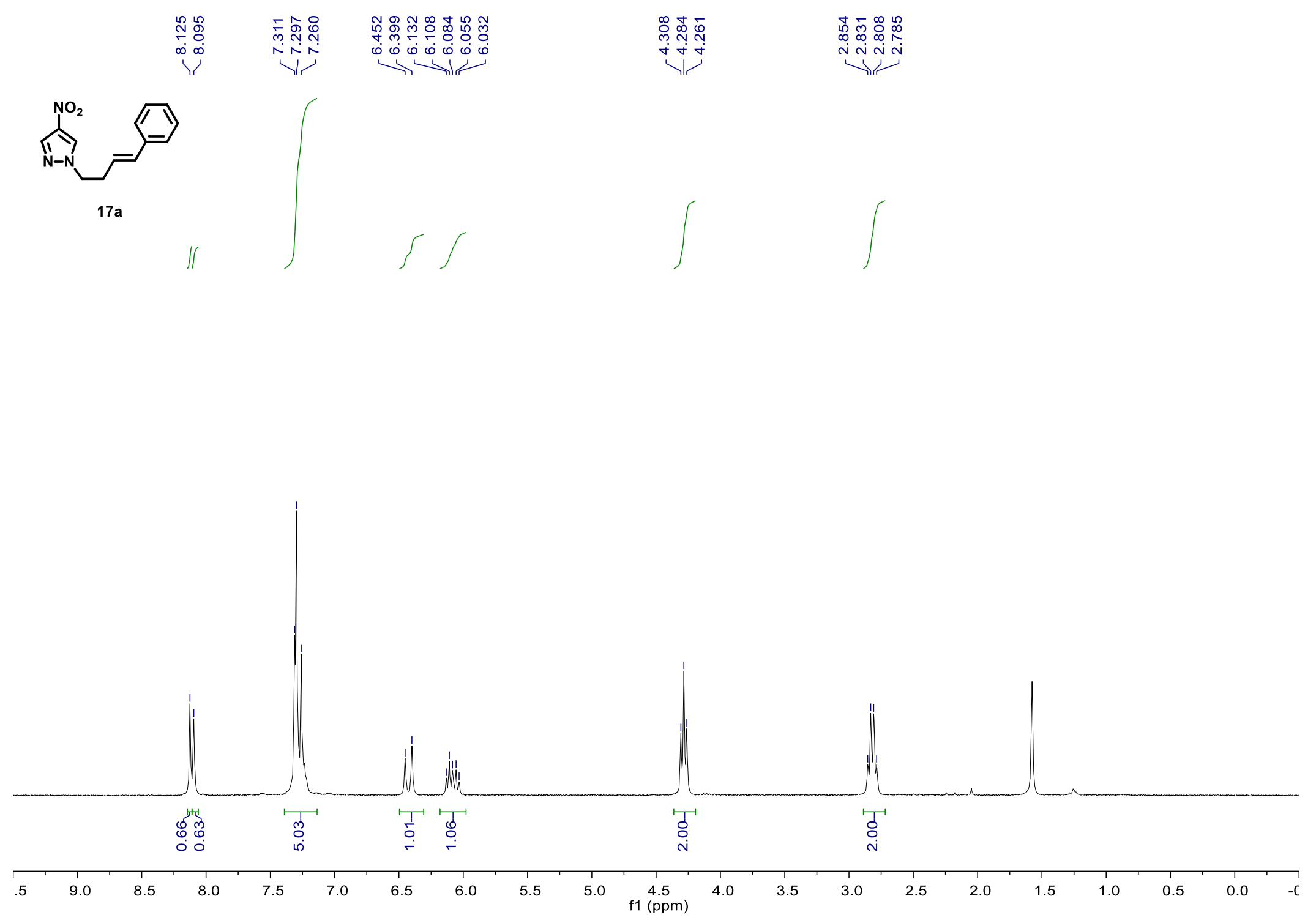



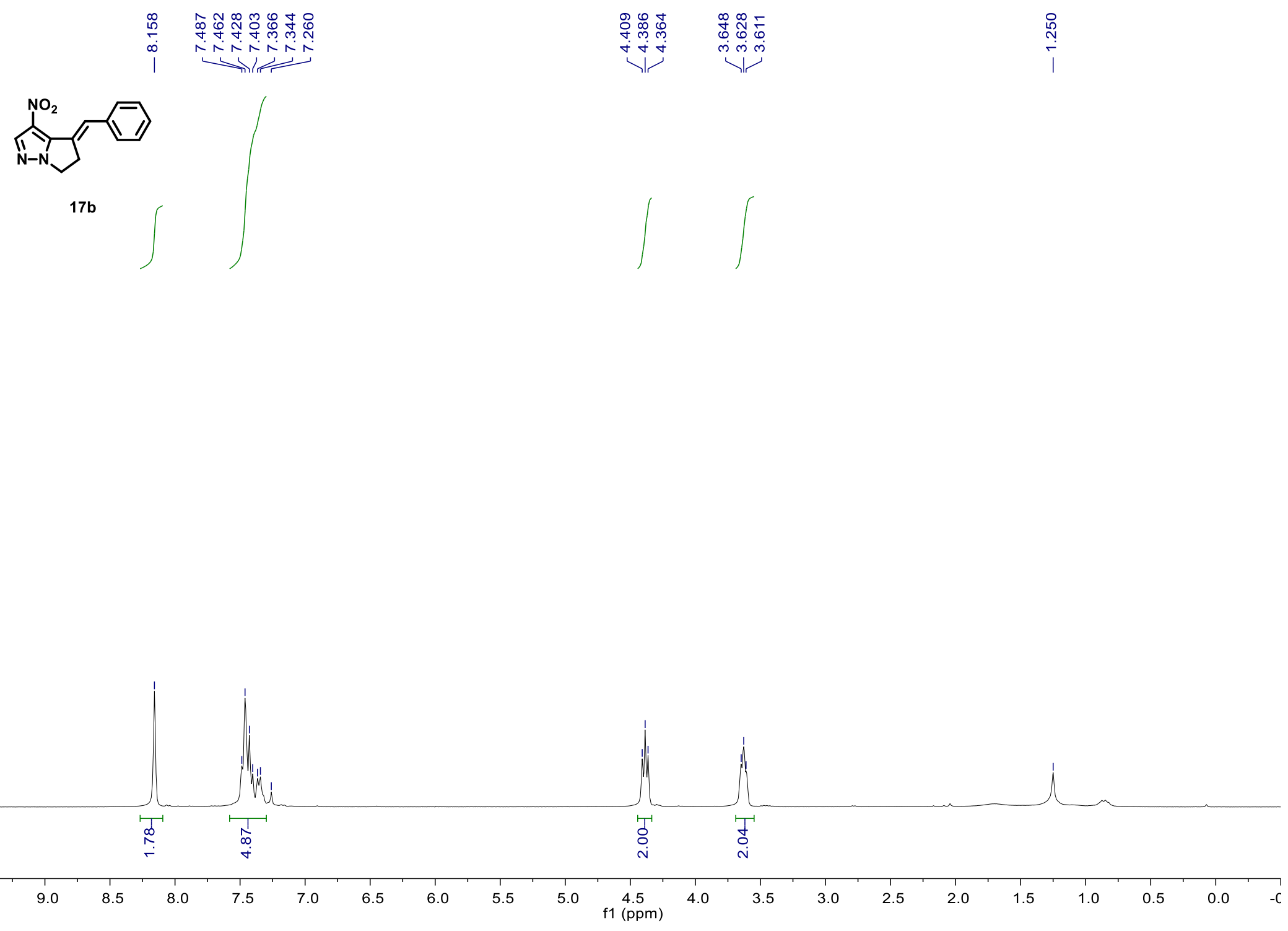



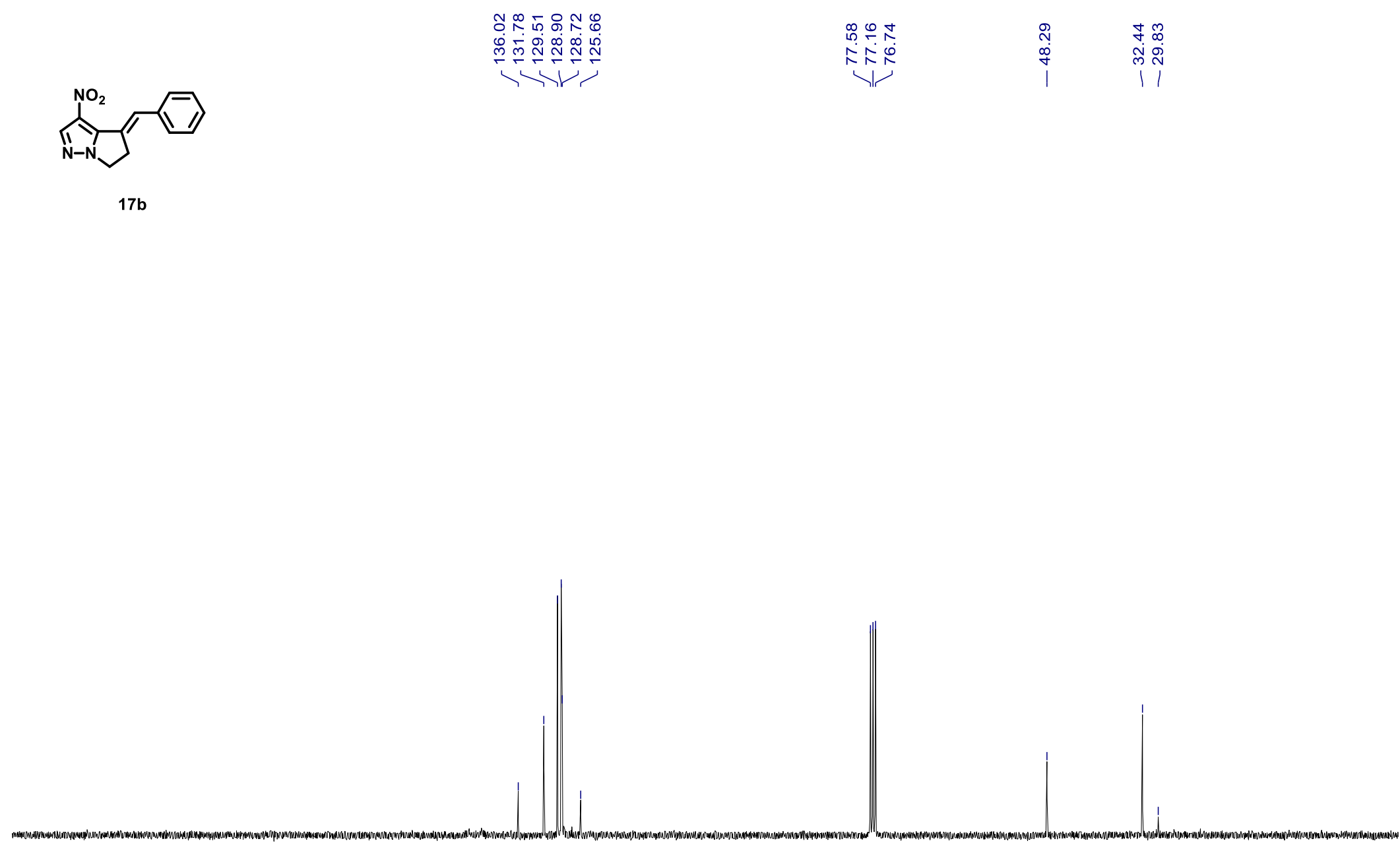

$\begin{array}{lllllllllllllllllllllllllllllll}210 & 200 & 190 & 180 & 170 & 160 & 150 & 140 & 130 & 120 & 110 & 100 & 90 & 80 & 70 & 60 & 50 & 40 & 30 & 20 & 10 & 0 & -\end{array}$ 

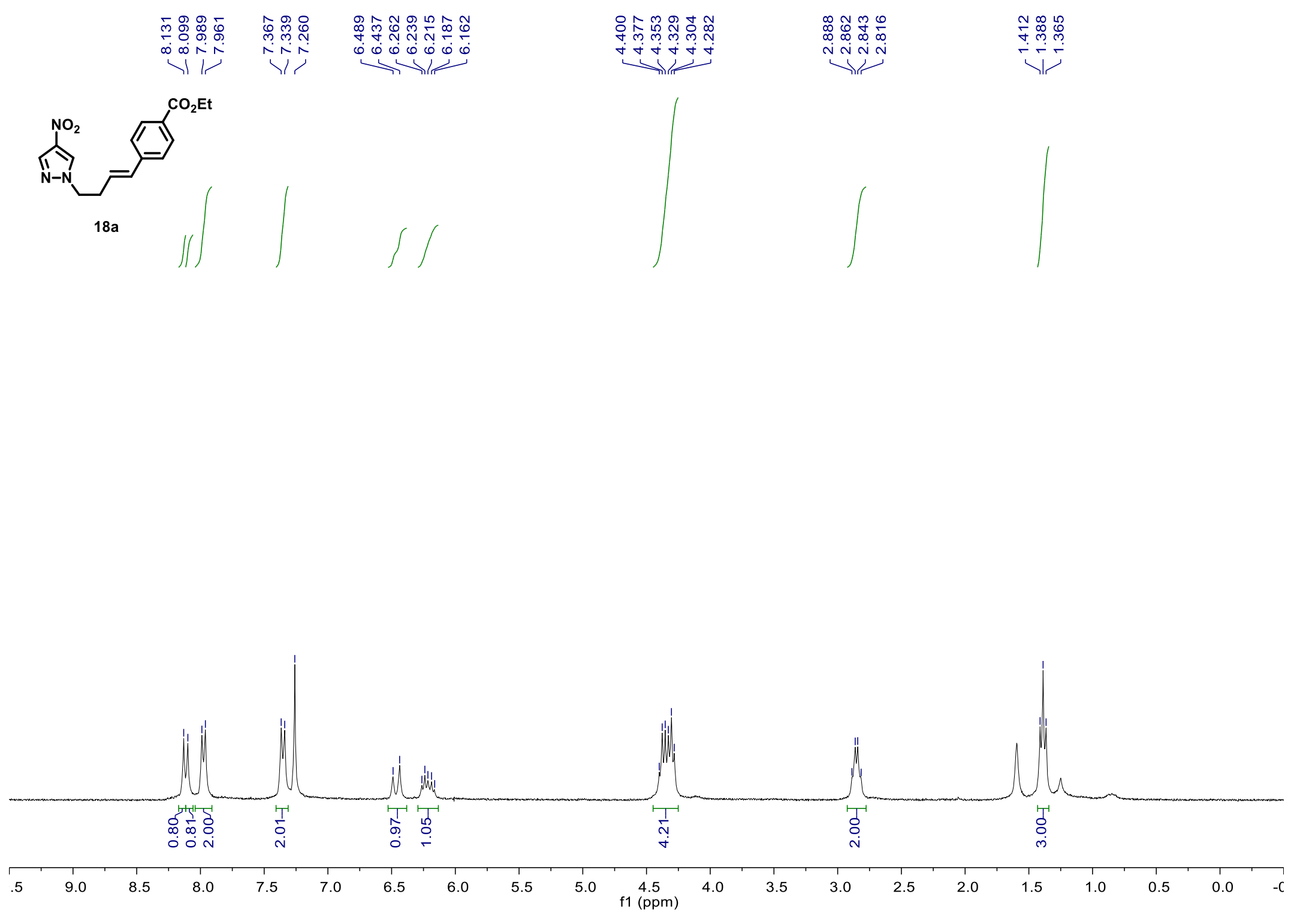


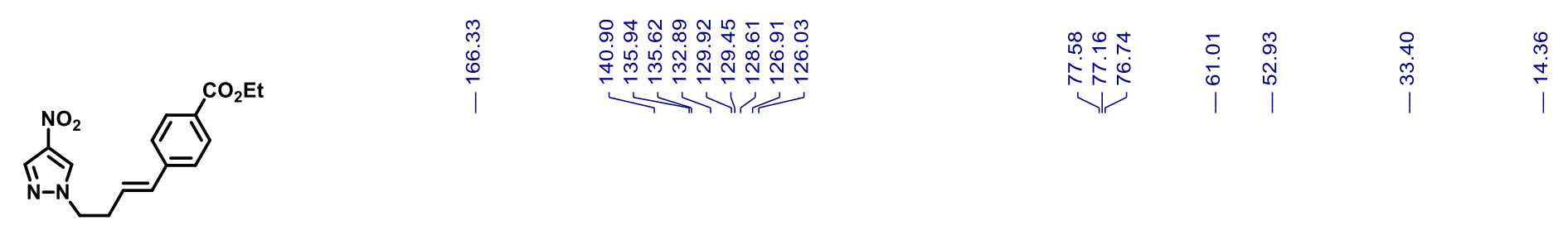

$18 \mathrm{a}$

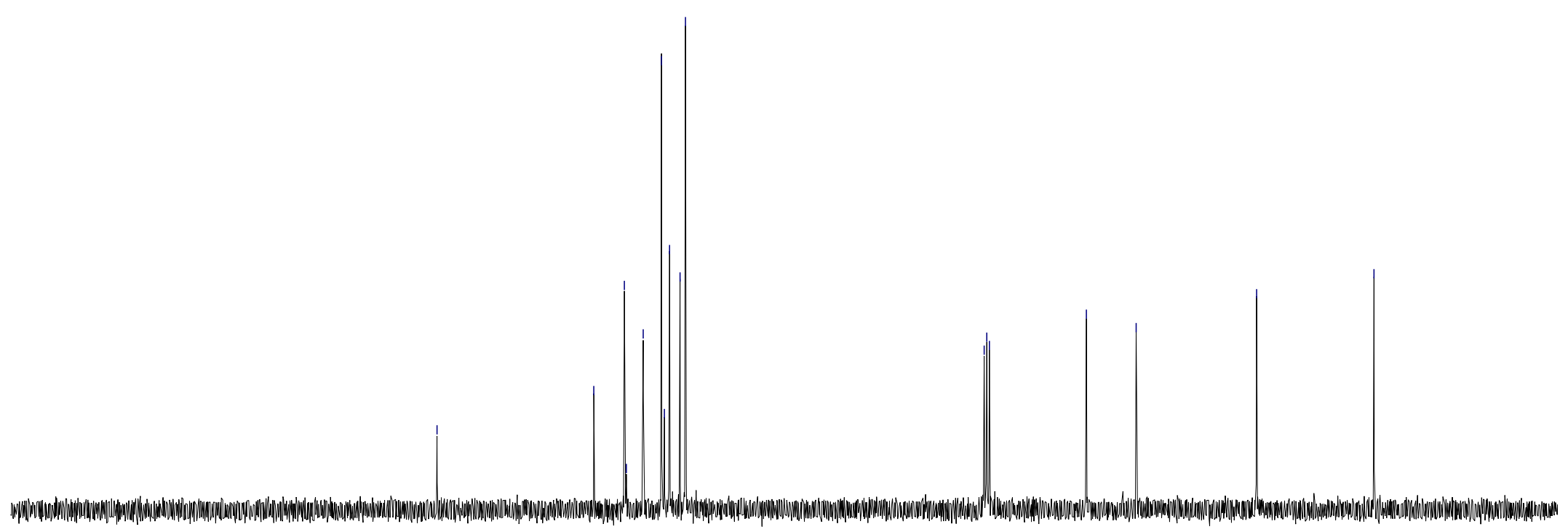

$\begin{array}{lllllllllllllllllllllllllllll}230 & 220 & 210 & 200 & 190 & 180 & 170 & 160 & 150 & 140 & 130 & 120 & 110 & 100 & 90 & 80 & 70 & 60 & 50 & 40 & 30 & 20 & 10 & 0 & -10\end{array}$ 


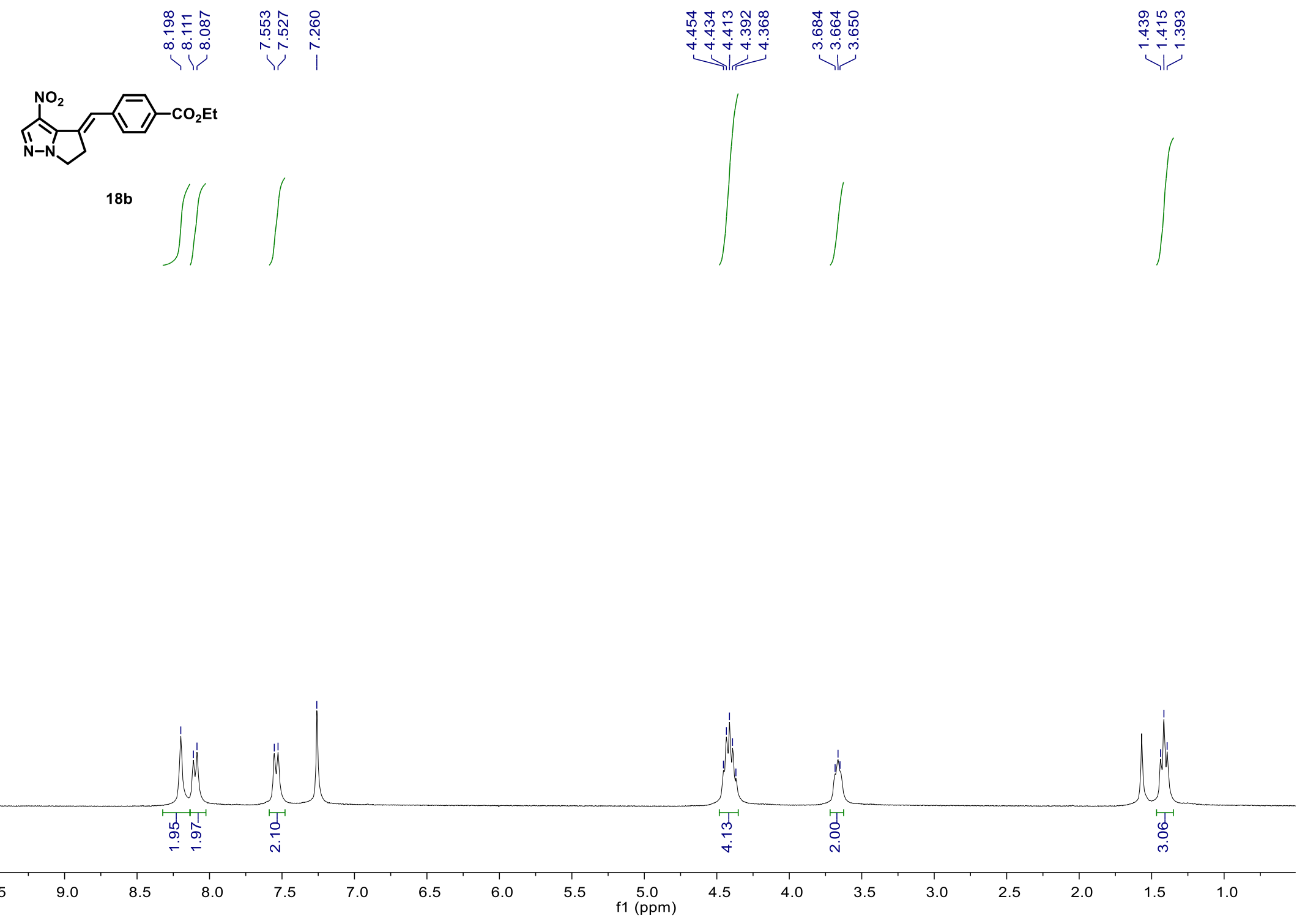




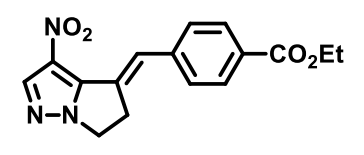

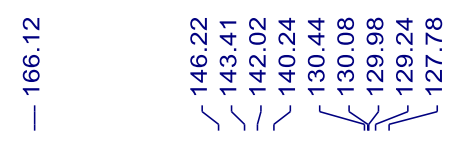

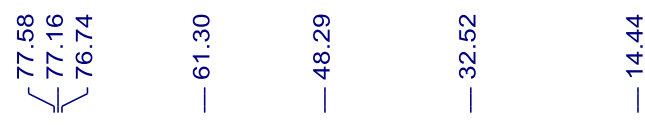

$18 \mathrm{~b}$

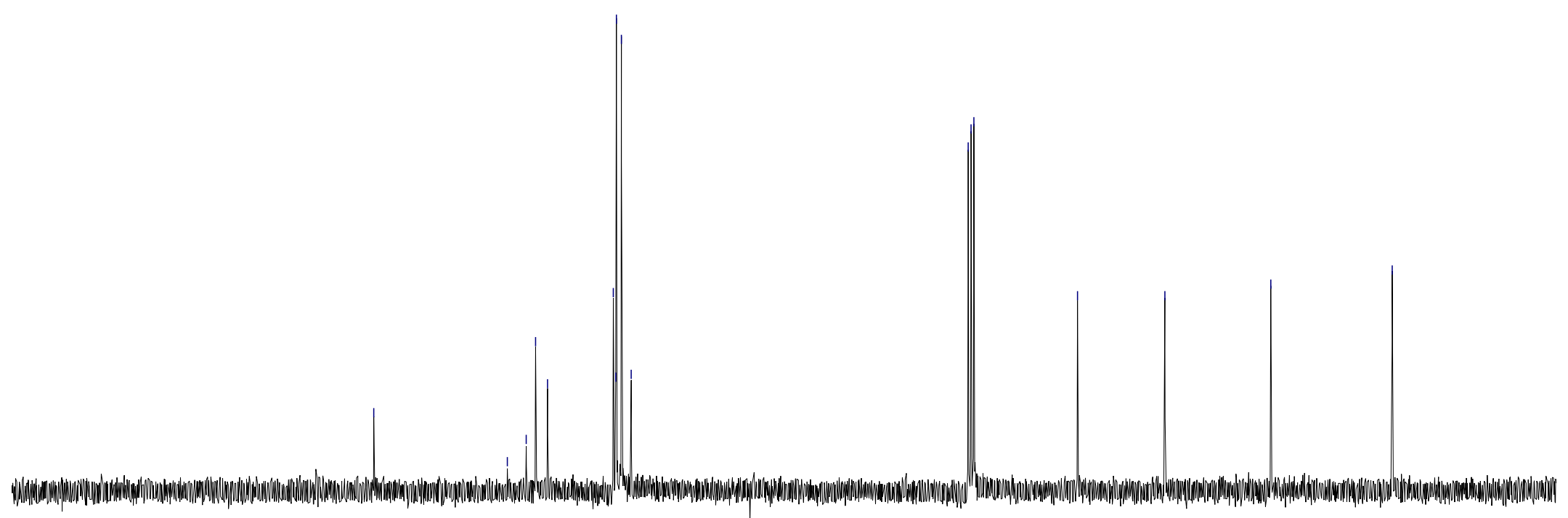

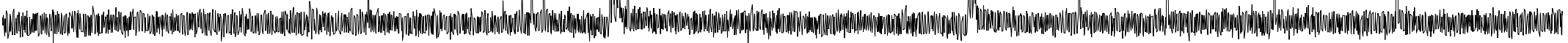

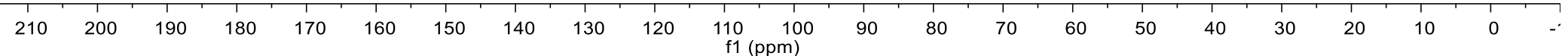




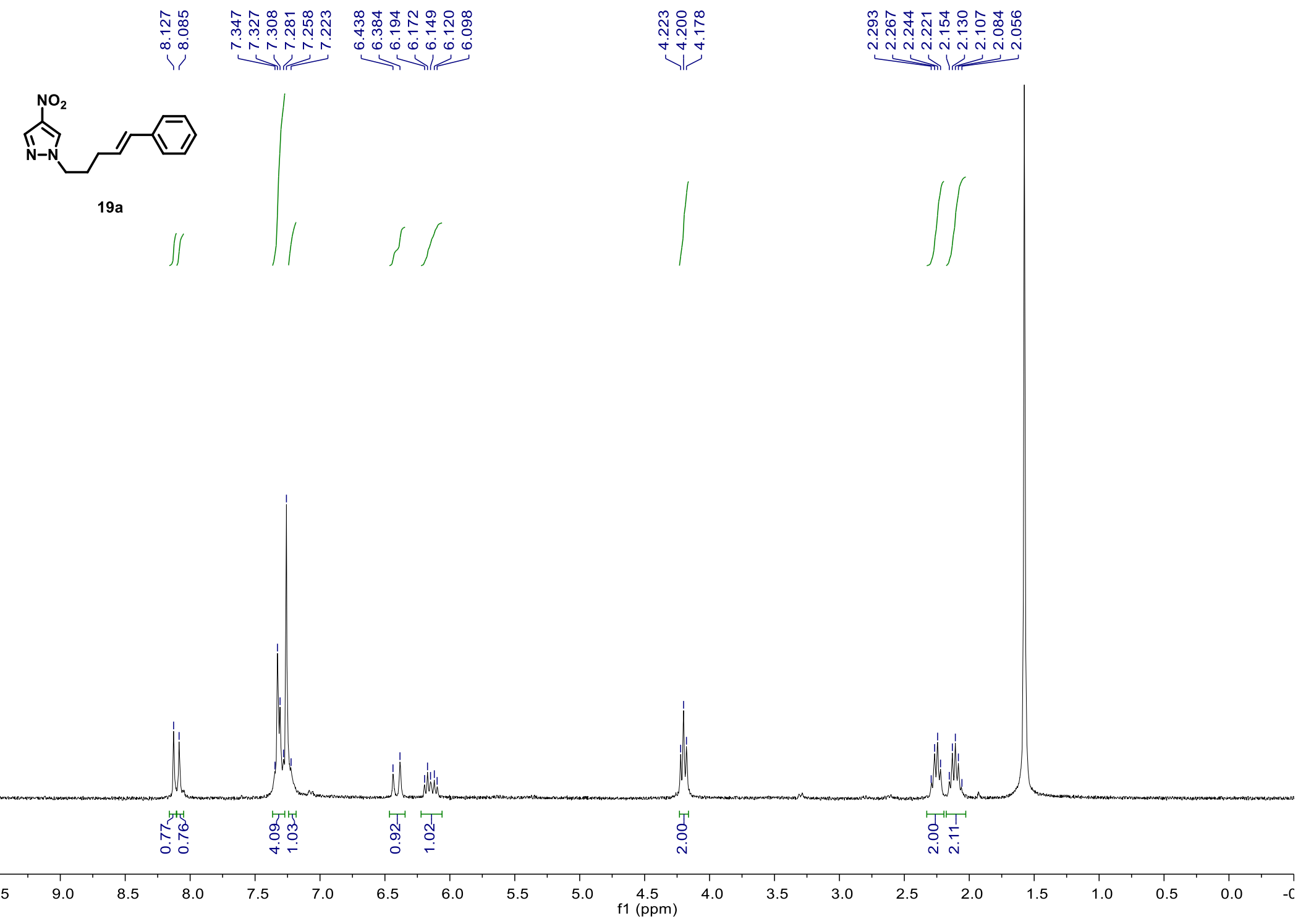




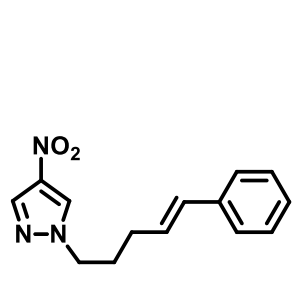

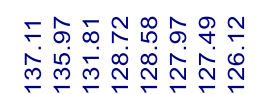

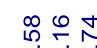

र당

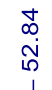

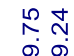

4

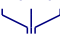

19a

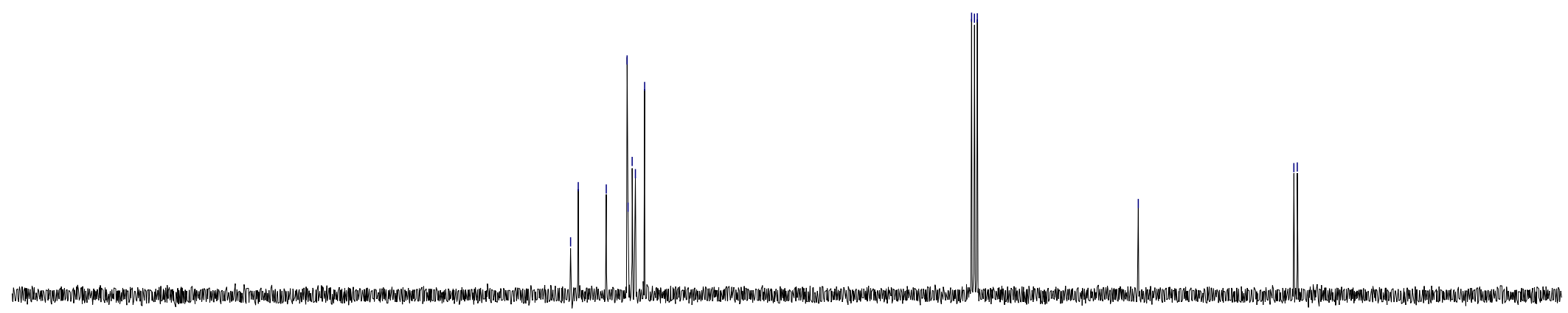

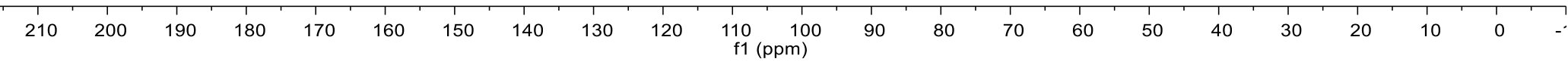




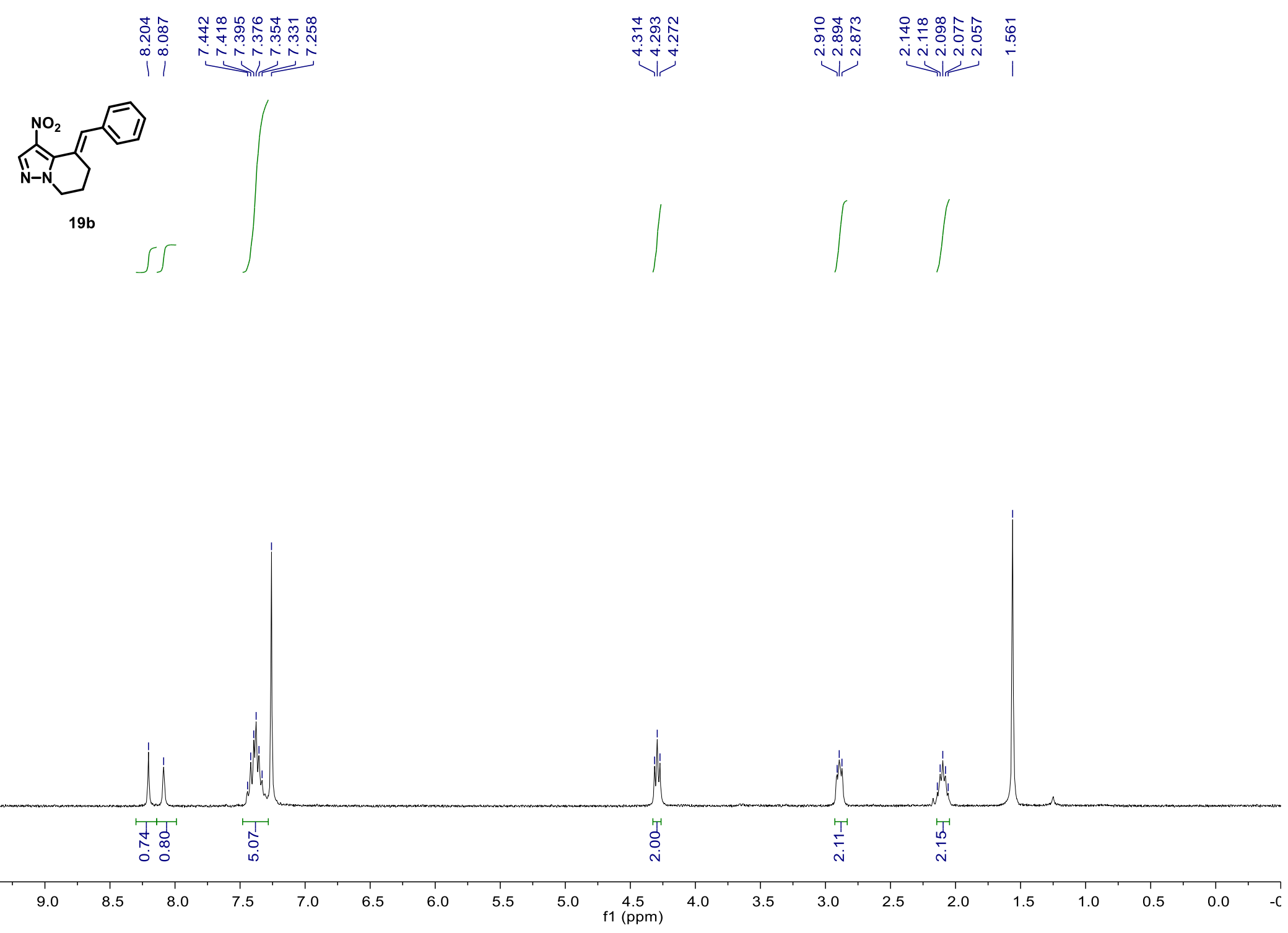




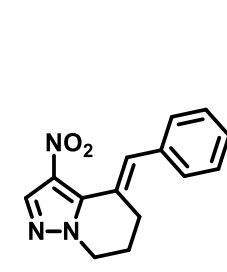

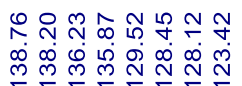

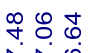

पi

$\checkmark$

N

$19 b$

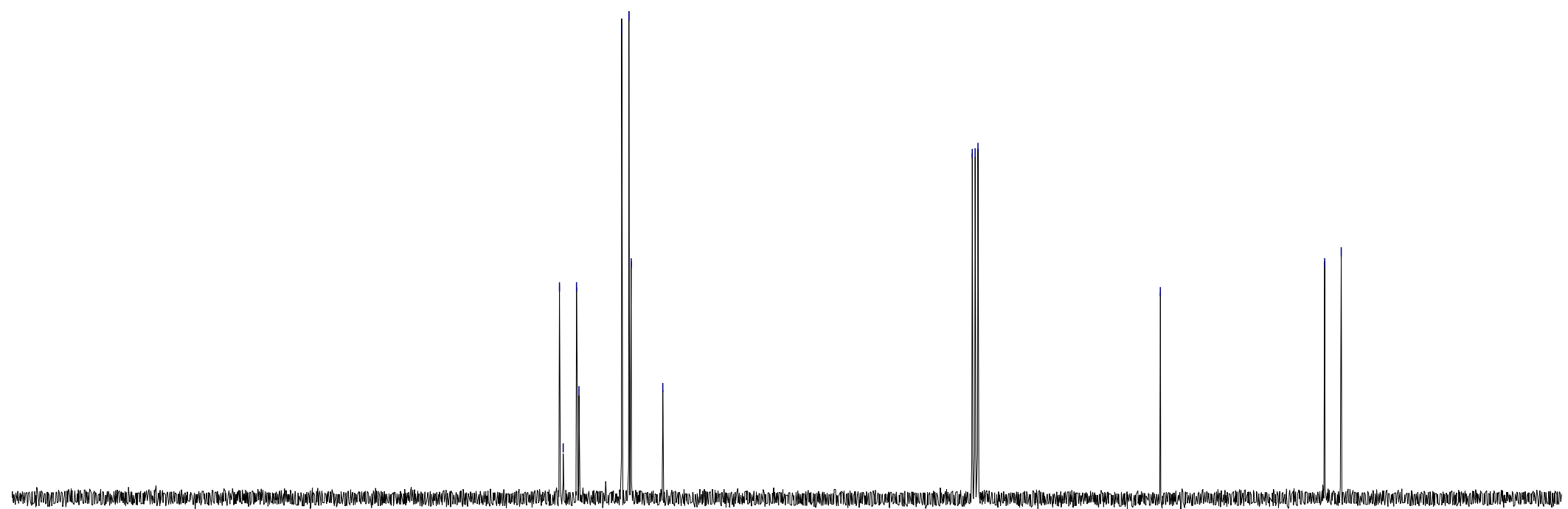

$\begin{array}{llllll}210 & 200 & 190 & 180 & 170 & 160\end{array}$

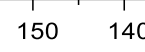

130

120

$110 \quad 100$
f1 $(\mathrm{ppm})$

$90 \quad 80$

$70 \quad 60$

50

30

20

10 

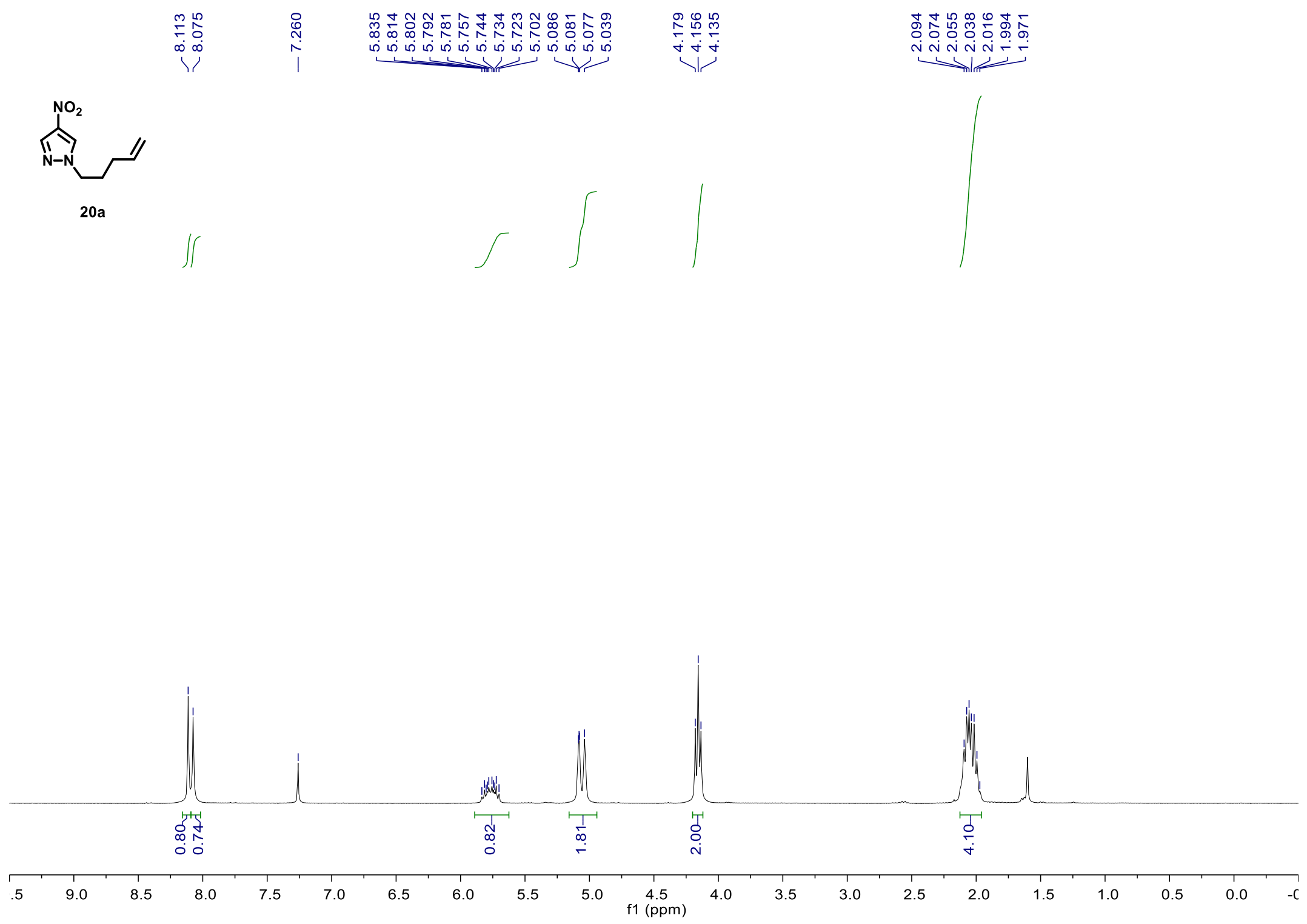

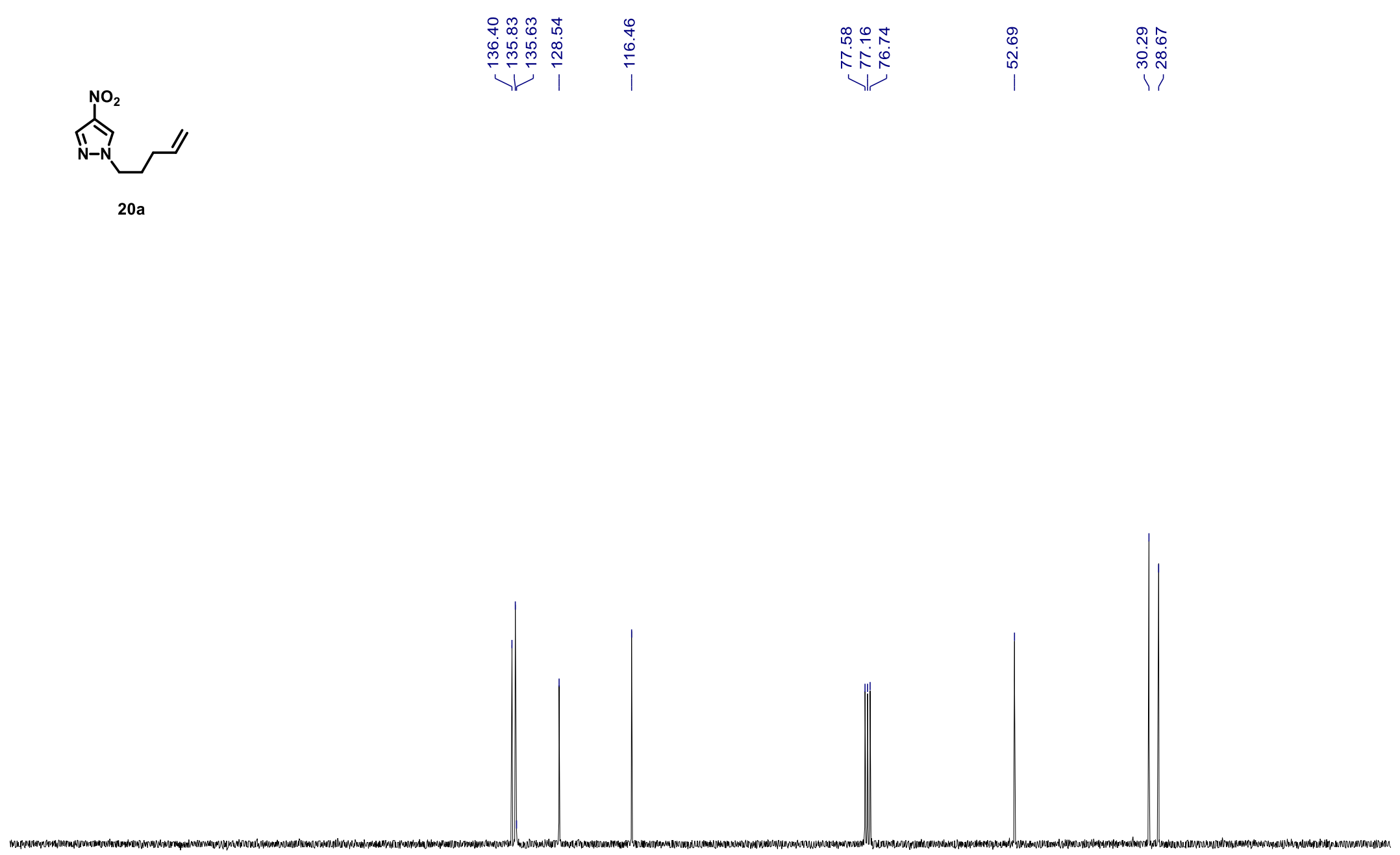


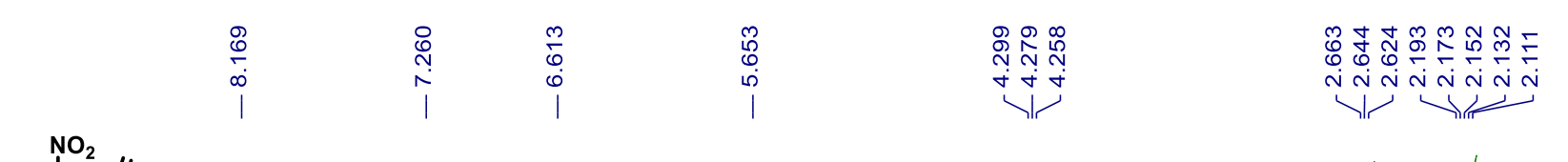

$\underbrace{\mathrm{NO}_{2}}_{\mathrm{N}-\mathrm{N}}$

20b
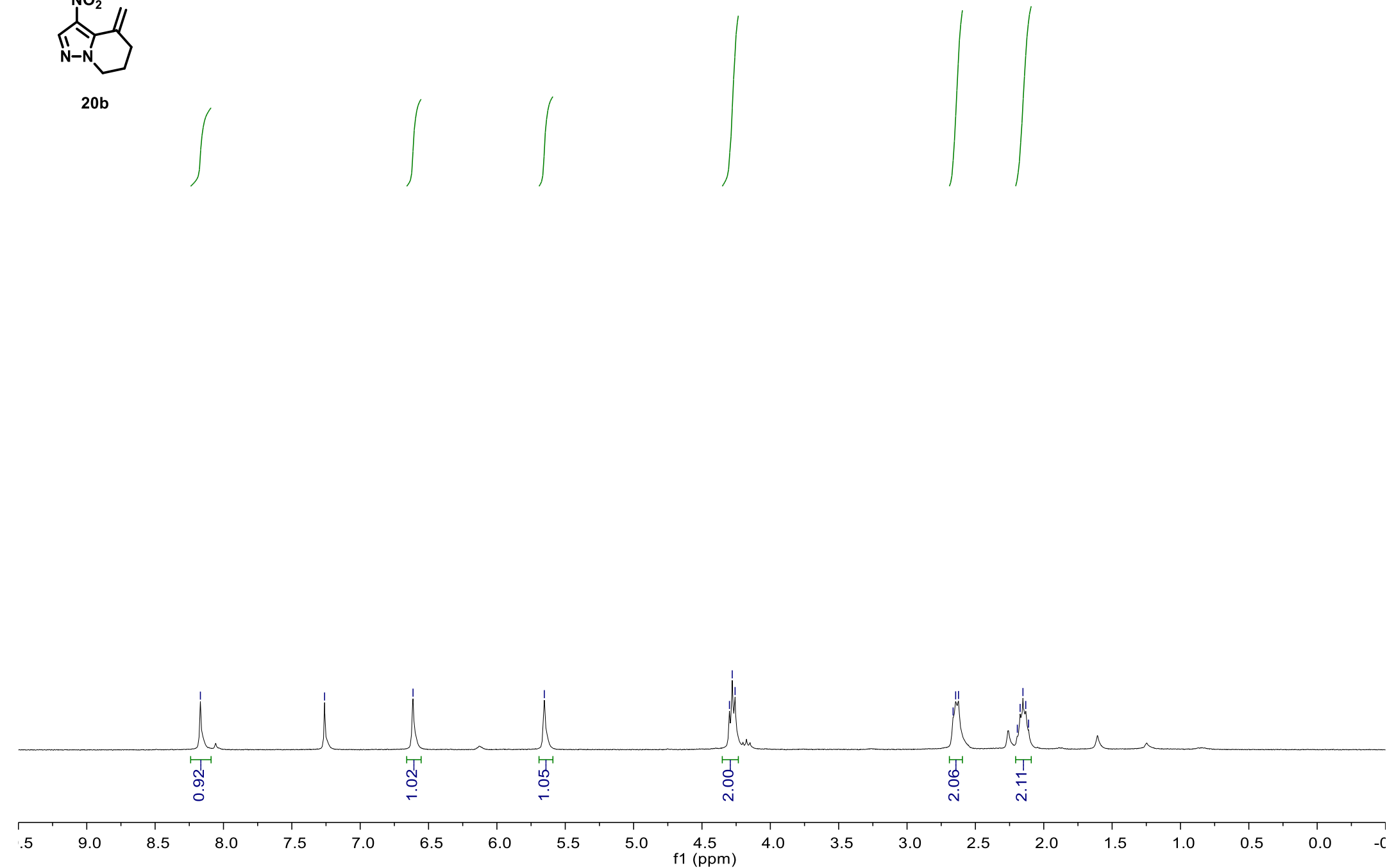


$\mathrm{NO}^{2}$

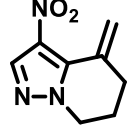
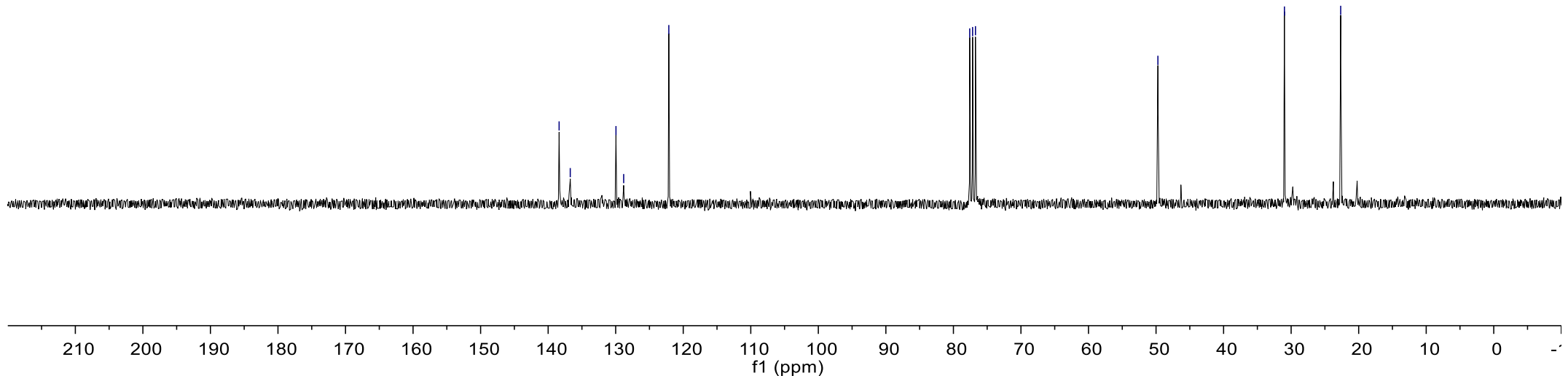

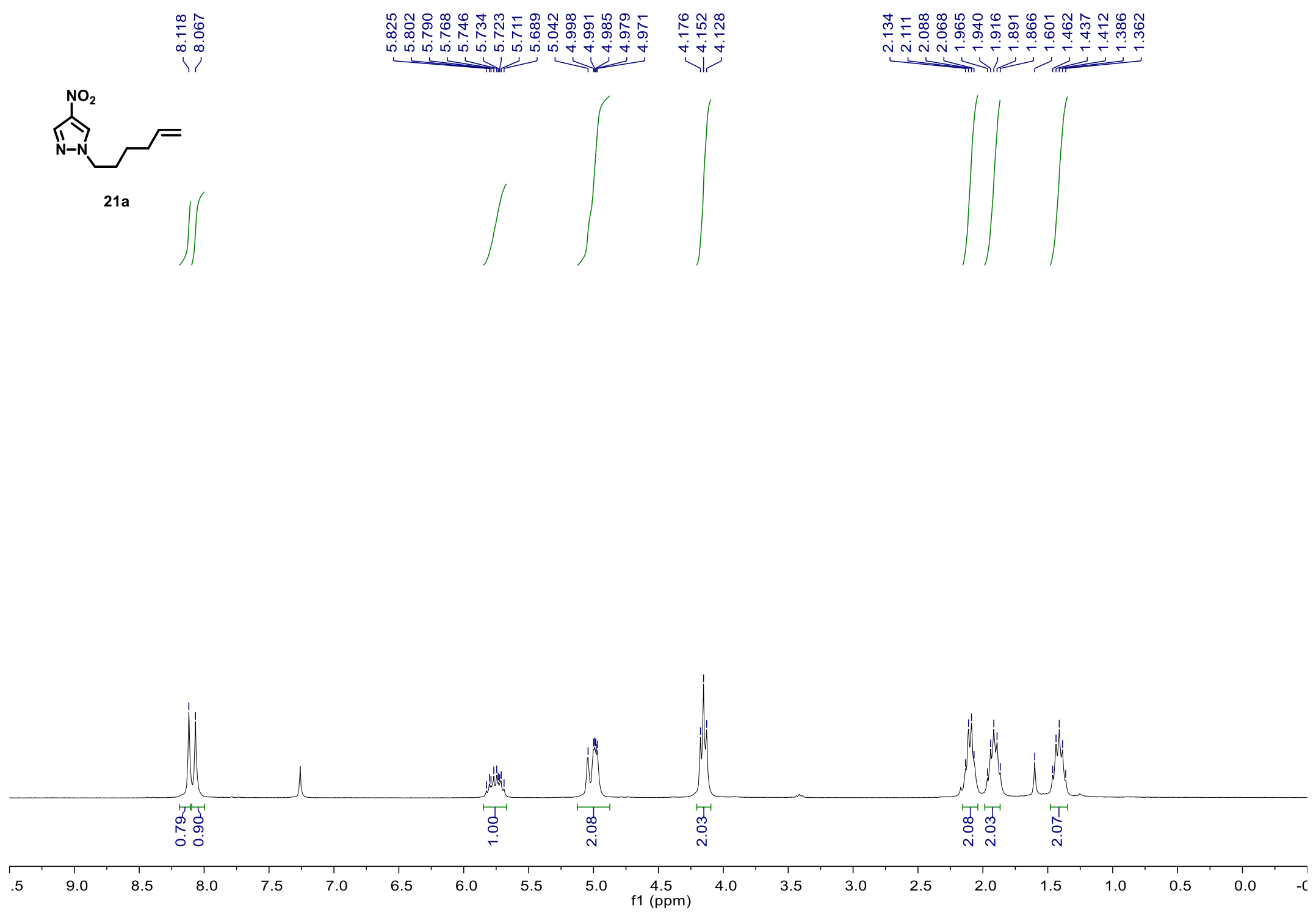


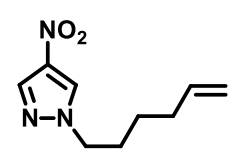

i

$21 a$

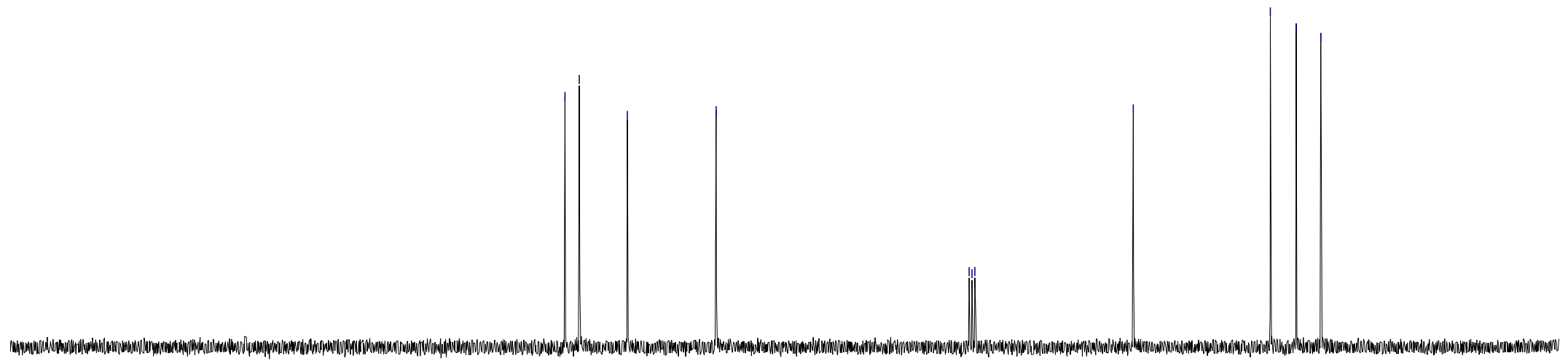

$\begin{array}{lll}180 & 170 \quad 160\end{array}$

150

130

$\begin{array}{llll}120 & 110 & 100 \quad 90\end{array}$

f1 (ppm)

90

70

60

50

40

20

100 


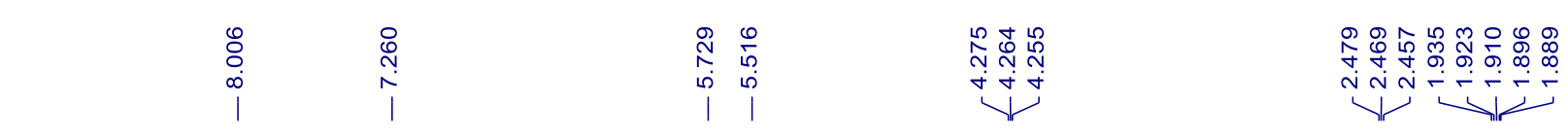

$\underbrace{\mathrm{NO}_{2}}_{\mathrm{N}-\mathrm{N}}$

21b
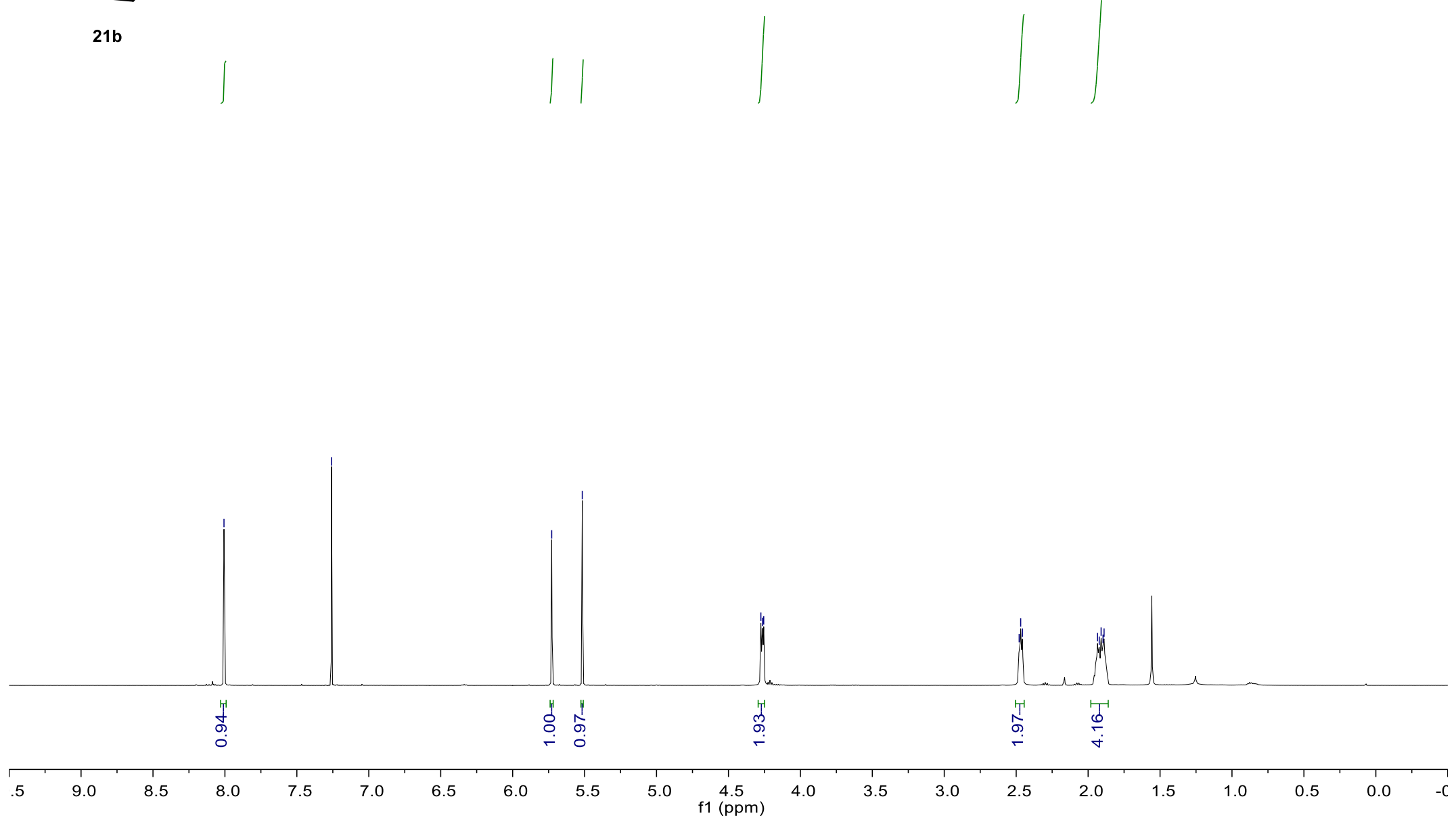


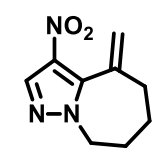

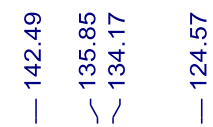

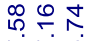

命定

$\stackrel{\substack{\sim \\ \dot{\sim}}}{i}$

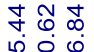

तl

21b

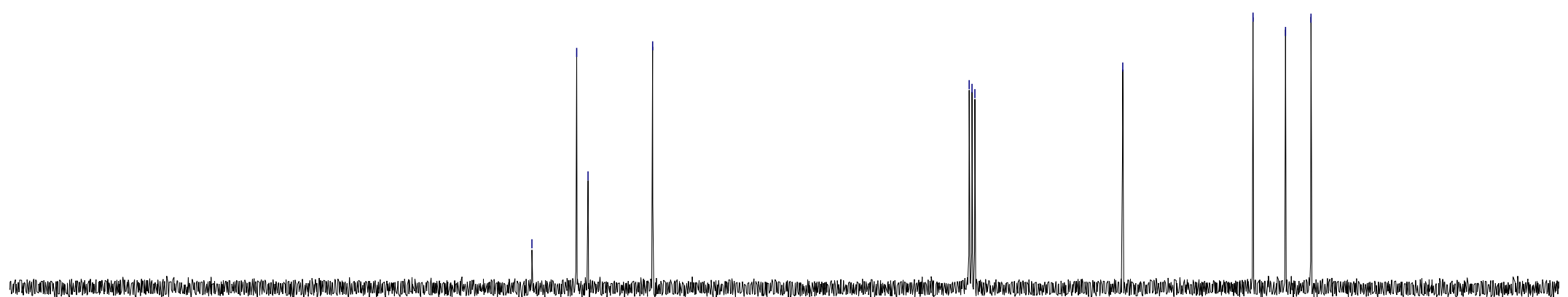

$\begin{array}{lllllllllll}210 & 200 & 190 & 180 & 170 & 160 & 150 & 140 & 130 & 120 & \begin{array}{c}110 \\ \mathrm{f} 1(\mathrm{ppm})\end{array}\end{array}$

90

80

$70 \quad 60$

$60 \quad 50$

40

$30 \quad 20 \quad 10 \quad 0$ 

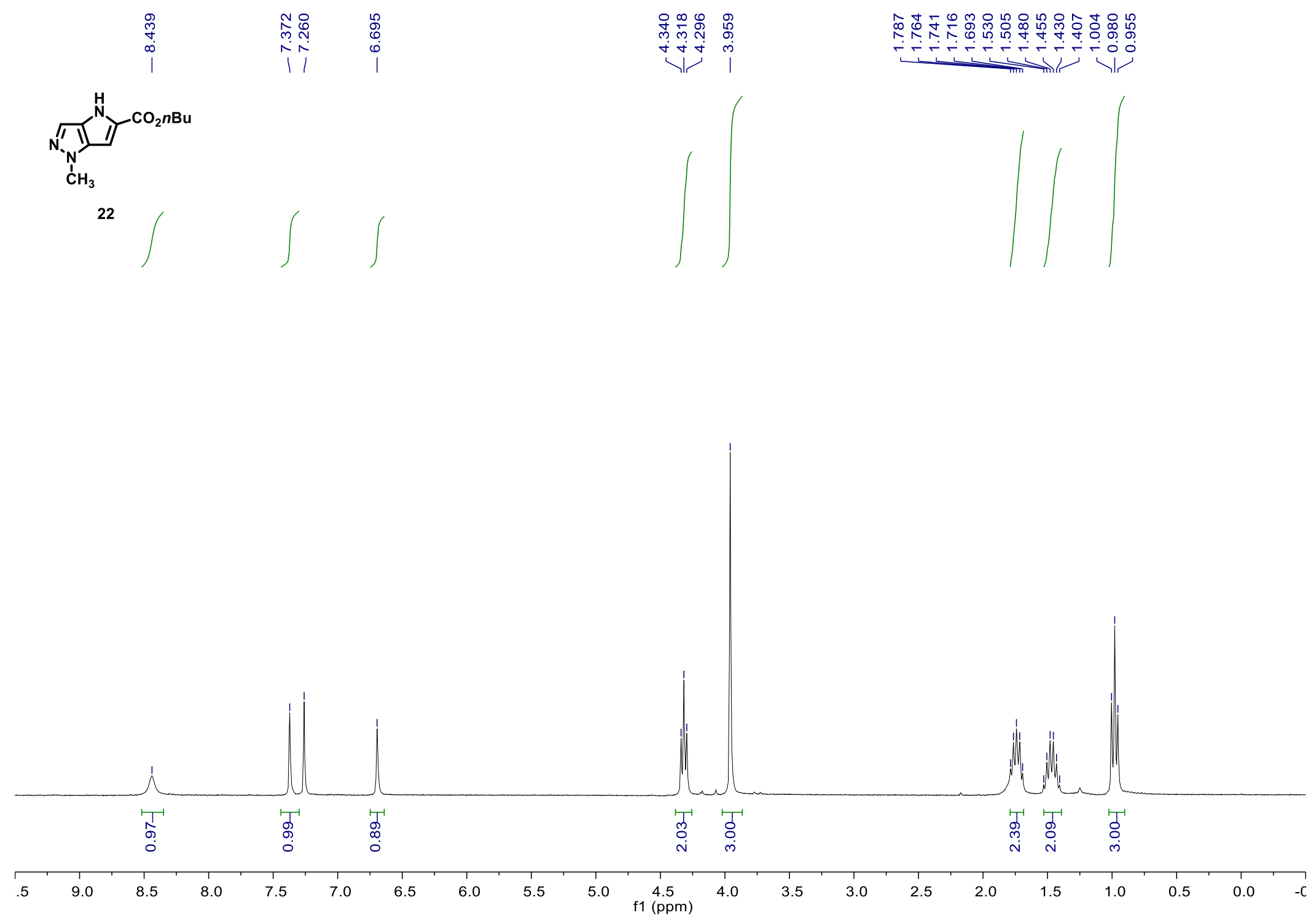


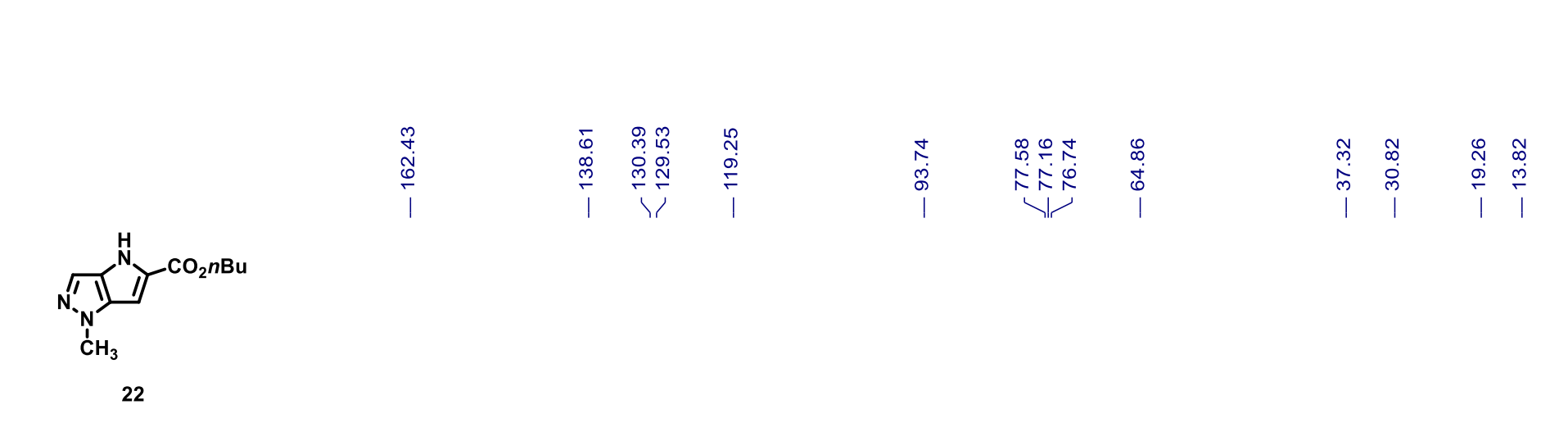



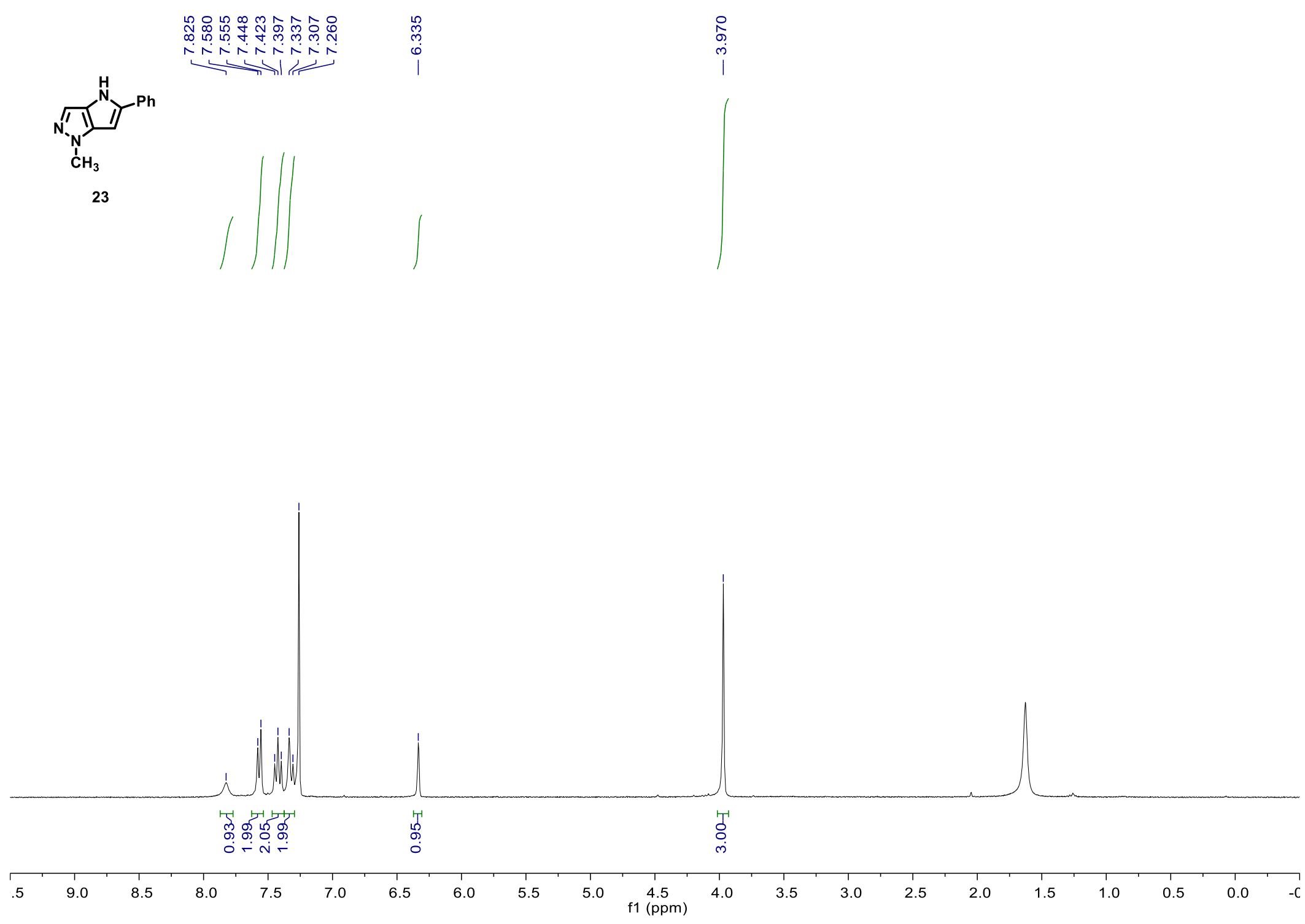


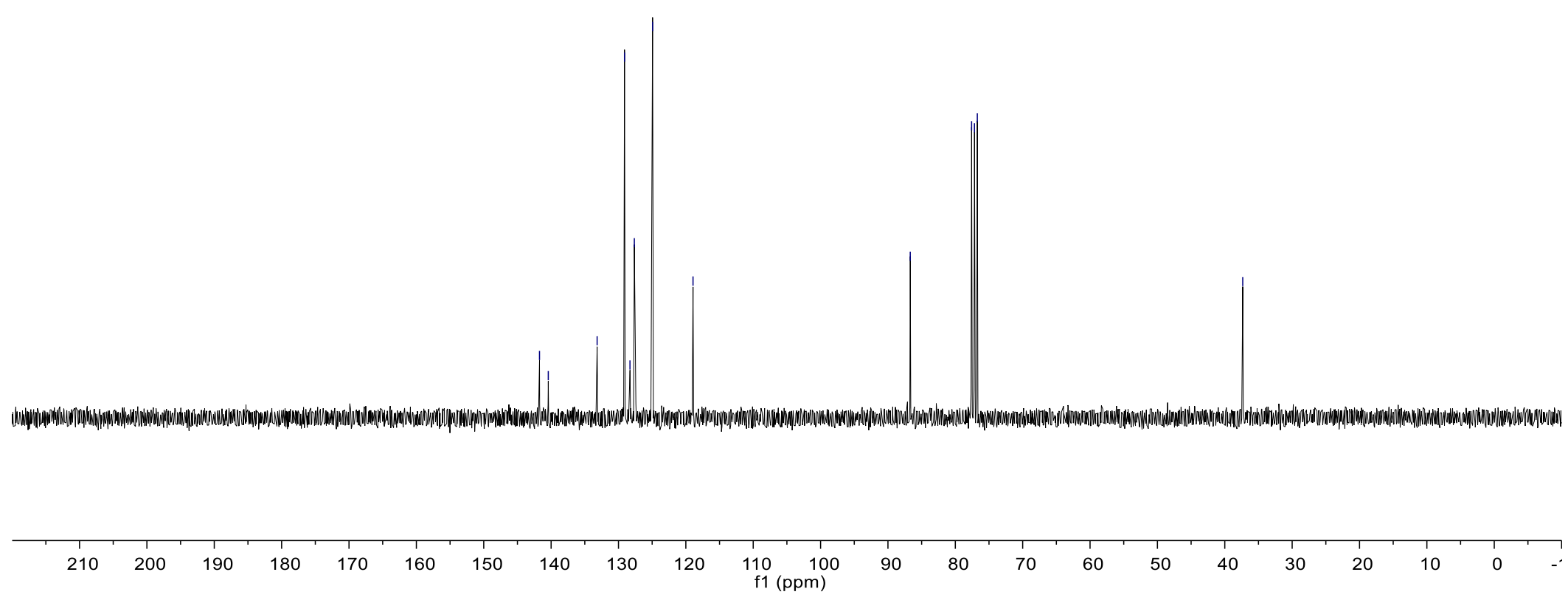




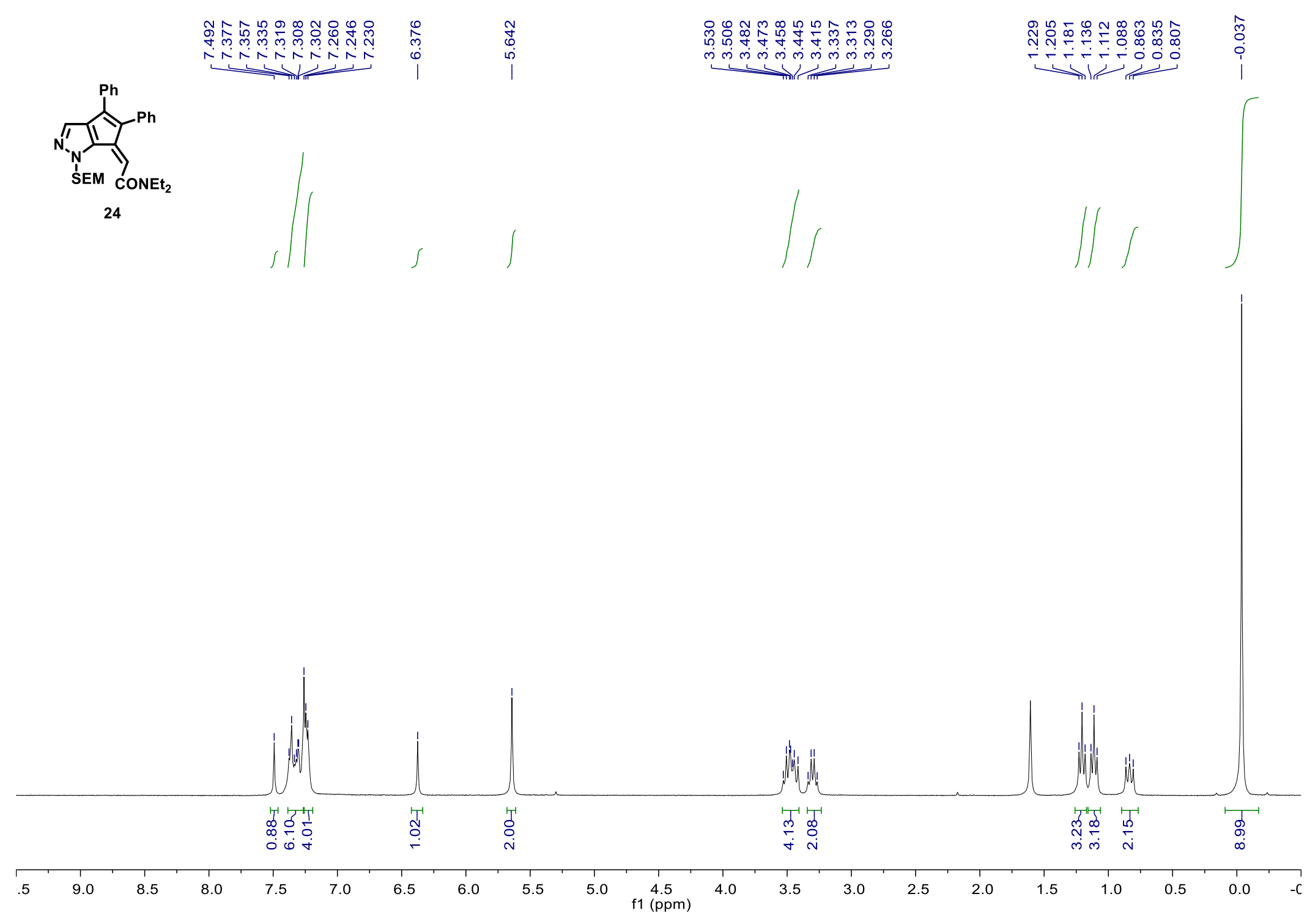




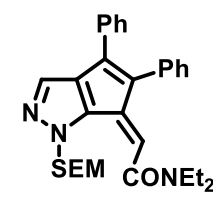

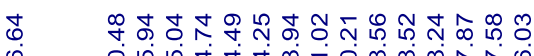

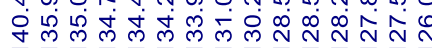

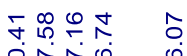

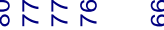

ำ

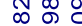

II I

용

IV

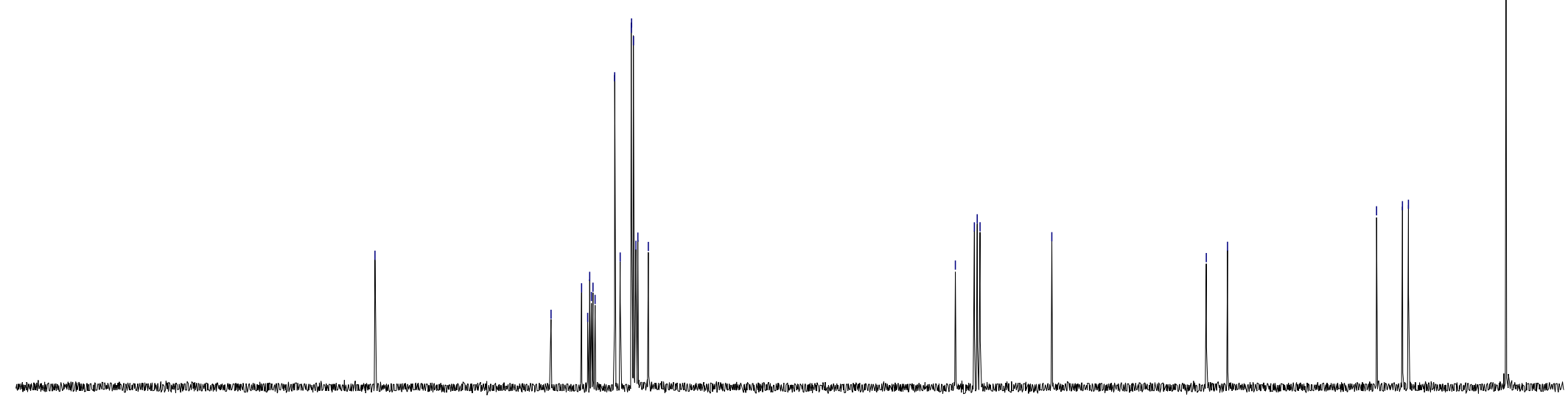

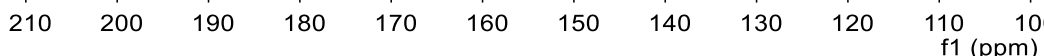

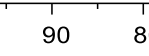

80
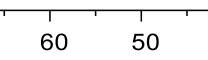


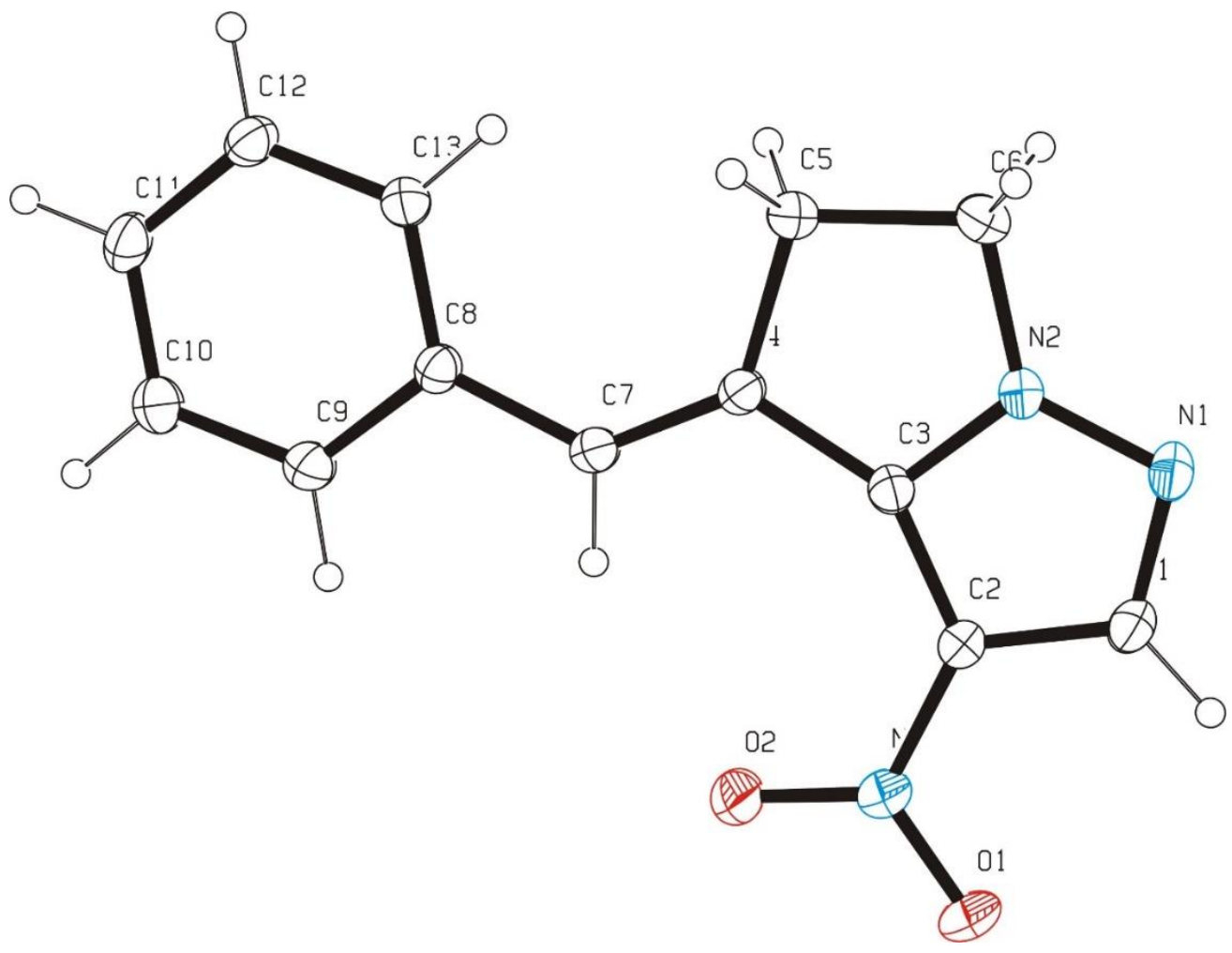

Figure S1. ORTEP diagram of $17 \mathrm{~b}$ with anisotropic displacement parameters at $30 \%$ probability

Table S1-1. Crystal data and structure refinement for $17 \mathrm{~b}$.

Identification code

Empirical formula

Formula weight

Temperature

Wavelength

Crystal system

Space group

Unit cell dimensions

Volume

$\mathrm{Z}$

Density (calculated)

Absorption coefficient
$17 \mathrm{~b}$

C13 H11 N3 O2

241.25

173(2) K

$0.71073 \AA$

Monoclinic

$\mathrm{P} 2(1) / \mathrm{n}$

$\mathrm{a}=6.5358(2) \AA \quad \mathrm{a}=90^{\circ}$.

$\mathrm{b}=15.1093(4) \AA$

$\mathrm{b}=90.698(2)^{\circ}$.

$\mathrm{c}=11.5347(4) \AA$

$\mathrm{g}=90^{\circ}$.

$1138.98(6) \AA^{3}$

4

$1.407 \mathrm{Mg} / \mathrm{m}^{3}$

$0.098 \mathrm{~mm}^{-1}$ 
$\mathrm{F}(000)$

Crystal size

Theta range for data collection

Index ranges

Reflections collected

Independent reflections

Completeness to theta $=26.00^{\circ}$

Absorption correction

Max. and min. transmission

Refinement method

Data / restraints / parameters

Goodness-of-fit on $\mathrm{F}^{2}$

Final $\mathrm{R}$ indices [I $>2 \operatorname{sigma}(\mathrm{I})]$

$\mathrm{R}$ indices (all data)

Largest diff. peak and hole
504

$0.36 \times 0.18 \times 0.18 \mathrm{~mm}^{3}$

2.22 to $26.00^{\circ}$.

$-8<=\mathrm{h}<=8,-18<=\mathrm{k}<=18,-13<=\mathrm{l}<=14$

8907

$2243[\mathrm{R}($ int $)=0.0290]$

$100.0 \%$

Semi-empirical from equivalents

0.9825 and 0.9654

Full-matrix least-squares on $\mathrm{F}^{2}$

2243 / 0 / 163

1.052

$\mathrm{R} 1=0.0453, \mathrm{wR} 2=0.1033$

$\mathrm{R} 1=0.0579, \mathrm{wR} 2=0.1116$

0.215 and -0.363 e. $\AA^{-3}$

Table S1-2. Atomic coordinates ( $\left.x 1^{4}\right)$ and equivalent isotropic displacement parameters $\left(\AA^{2} \times 10^{3}\right)$ for $17 \mathrm{~b} . \mathrm{U}(\mathrm{eq})$ is defined as one third of the trace of the orthogonalized $U^{i j}$ tensor.

\begin{tabular}{lrccl}
\hline & $\mathrm{x}$ & $\mathrm{y}$ & $\mathrm{z}$ & $\mathrm{U}(\mathrm{eq})$ \\
\hline $\mathrm{O}(1)$ & $2596(2)$ & $5613(1)$ & $4111(1)$ & $63(1)$ \\
$\mathrm{O}(2)$ & $-247(2)$ & $5424(1)$ & $3186(1)$ & $57(1)$ \\
$\mathrm{N}(1)$ & $3111(2)$ & $2928(1)$ & $4273(1)$ & $37(1)$ \\
$\mathrm{N}(2)$ & $1359(2)$ & $2788(1)$ & $3662(1)$ & $30(1)$ \\
$\mathrm{N}(3)$ & $1265(2)$ & $5130(1)$ & $3697(1)$ & $40(1)$ \\
$\mathrm{C}(1)$ & $3202(2)$ & $3797(1)$ & $4359(1)$ & $37(1)$ \\
$\mathrm{C}(2)$ & $1530(2)$ & $4204(1)$ & $3809(1)$ & $32(1)$ \\
$\mathrm{C}(3)$ & $325(2)$ & $3517(1)$ & $3355(1)$ & $28(1)$ \\
$\mathrm{C}(4)$ & $-1504(2)$ & $3272(1)$ & $2704(1)$ & $26(1)$ \\
$\mathrm{C}(5)$ & $-1459(2)$ & $2266(1)$ & $2614(1)$ & $32(1)$ \\
$\mathrm{C}(6)$ & $498(2)$ & $1956(1)$ & $3252(1)$ & $34(1)$ \\
$\mathrm{C}(7)$ & $-2886(2)$ & $3850(1)$ & $2287(1)$ & $30(1)$ \\
$\mathrm{C}(8)$ & $-4768(2)$ & $3703(1)$ & $1620(1)$ & $28(1)$
\end{tabular}




$\begin{array}{lrrrr}\mathrm{C}(9) & -5844(2) & 4460(1) & 1275(2) & 38(1) \\ \mathrm{C}(10) & -7644(2) & 4413(1) & 644(2) & 43(1) \\ \mathrm{C}(11) & -8454(2) & 3598(1) & 345(2) & 38(1) \\ \mathrm{C}(12) & -7418(2) & 2840(1) & 673(1) & 34(1) \\ \mathrm{C}(13) & -5606(2) & 2885(1) & 1301(1) & 31(1)\end{array}$

Table S1-3. Bond lengths $[\AA ̊ \AA]$ and angles $\left[{ }^{\circ}\right]$ for $17 \mathrm{~b}$.

\begin{tabular}{ll}
\hline $\mathrm{O}(1)-\mathrm{N}(3)$ & $1.2276(17)$ \\
$\mathrm{O}(2)-\mathrm{N}(3)$ & $1.2284(17)$ \\
$\mathrm{N}(1)-\mathrm{C}(1)$ & $1.3187(19)$ \\
$\mathrm{N}(1)-\mathrm{N}(2)$ & $1.3539(17)$ \\
$\mathrm{N}(2)-\mathrm{C}(3)$ & $1.3388(18)$ \\
$\mathrm{N}(2)-\mathrm{C}(6)$ & $1.4532(18)$ \\
$\mathrm{N}(3)-\mathrm{C}(2)$ & $1.416(2)$ \\
$\mathrm{C}(1)-\mathrm{C}(2)$ & $1.398(2)$ \\
$\mathrm{C}(1)-\mathrm{H}(1 \mathrm{~A})$ & 0.9500 \\
$\mathrm{C}(2)-\mathrm{C}(3)$ & $1.400(2)$ \\
$\mathrm{C}(3)-\mathrm{C}(4)$ & $1.452(2)$ \\
$\mathrm{C}(4)-\mathrm{C}(7)$ & $1.342(2)$ \\
$\mathrm{C}(4)-\mathrm{C}(5)$ & $1.5244(19)$ \\
$\mathrm{C}(5)-\mathrm{C}(6)$ & $1.540(2)$ \\
$\mathrm{C}(5)-\mathrm{H}(5 \mathrm{~A})$ & 0.9900 \\
$\mathrm{C}(5)-\mathrm{H}(5 \mathrm{~B})$ & 0.9900 \\
$\mathrm{C}(6)-\mathrm{H}(6 \mathrm{~A})$ & 0.9900 \\
$\mathrm{C}(6)-\mathrm{H}(6 \mathrm{~B})$ & 0.9900 \\
$\mathrm{C}(7)-\mathrm{C}(8)$ & $1.460(2)$ \\
$\mathrm{C}(7)-\mathrm{H}(7 \mathrm{~A})$ & 0.9500 \\
$\mathrm{C}(8)-\mathrm{C}(9)$ & $1.397(2)$ \\
$\mathrm{C}(8)-\mathrm{C}(13)$ & $1.400(2)$ \\
$\mathrm{C}(9)-\mathrm{C}(10)$ & $1.378(2)$ \\
$\mathrm{C}(9)-\mathrm{H}(9 \mathrm{~A})$ & 0.9500 \\
$\mathrm{C}(10)-\mathrm{C}(11)$ & $1.382(2)$ \\
$\mathrm{C}(10)-\mathrm{H}(10 \mathrm{~A})$ & 0.9500 \\
& \\
& \\
&
\end{tabular}




\begin{tabular}{|c|c|}
\hline $\mathrm{C}(11)-\mathrm{C}(12)$ & $1.381(2)$ \\
\hline $\mathrm{C}(11)-\mathrm{H}(11 \mathrm{~A})$ & 0.9500 \\
\hline $\mathrm{C}(12)-\mathrm{C}(13)$ & $1.383(2)$ \\
\hline $\mathrm{C}(12)-\mathrm{H}(12 \mathrm{~A})$ & 0.9500 \\
\hline $\mathrm{C}(13)-\mathrm{H}(13 \mathrm{~A})$ & 0.9500 \\
\hline $\mathrm{C}(1)-\mathrm{N}(1)-\mathrm{N}(2)$ & $103.42(12)$ \\
\hline $\mathrm{C}(3)-\mathrm{N}(2)-\mathrm{N}(1)$ & $115.45(11)$ \\
\hline $\mathrm{C}(3)-\mathrm{N}(2)-\mathrm{C}(6)$ & $115.69(12)$ \\
\hline $\mathrm{N}(1)-\mathrm{N}(2)-\mathrm{C}(6)$ & $128.80(11)$ \\
\hline $\mathrm{O}(1)-\mathrm{N}(3)-\mathrm{O}(2)$ & $122.23(14)$ \\
\hline $\mathrm{O}(1)-\mathrm{N}(3)-\mathrm{C}(2)$ & $117.84(14)$ \\
\hline $\mathrm{O}(2)-\mathrm{N}(3)-\mathrm{C}(2)$ & $119.92(13)$ \\
\hline $\mathrm{N}(1)-\mathrm{C}(1)-\mathrm{C}(2)$ & $111.65(14)$ \\
\hline $\mathrm{N}(1)-\mathrm{C}(1)-\mathrm{H}(1 \mathrm{~A})$ & 124.2 \\
\hline $\mathrm{C}(2)-\mathrm{C}(1)-\mathrm{H}(1 \mathrm{~A})$ & 124.2 \\
\hline$C(1)-C(2)-C(3)$ & $106.12(13)$ \\
\hline $\mathrm{C}(1)-\mathrm{C}(2)-\mathrm{N}(3)$ & $124.68(14)$ \\
\hline $\mathrm{C}(3)-\mathrm{C}(2)-\mathrm{N}(3)$ & $129.08(13)$ \\
\hline $\mathrm{N}(2)-\mathrm{C}(3)-\mathrm{C}(2)$ & $103.36(13)$ \\
\hline $\mathrm{N}(2)-\mathrm{C}(3)-\mathrm{C}(4)$ & $109.65(12)$ \\
\hline$C(2)-C(3)-C(4)$ & $146.99(13)$ \\
\hline $\mathrm{C}(7)-\mathrm{C}(4)-\mathrm{C}(3)$ & $124.47(13)$ \\
\hline$C(7)-C(4)-C(5)$ & $129.67(13)$ \\
\hline$C(3)-C(4)-C(5)$ & $105.86(11)$ \\
\hline$C(4)-C(5)-C(6)$ & $106.67(11)$ \\
\hline $\mathrm{C}(4)-\mathrm{C}(5)-\mathrm{H}(5 \mathrm{~A})$ & 110.4 \\
\hline $\mathrm{C}(6)-\mathrm{C}(5)-\mathrm{H}(5 \mathrm{~A})$ & 110.4 \\
\hline $\mathrm{C}(4)-\mathrm{C}(5)-\mathrm{H}(5 \mathrm{~B})$ & 110.4 \\
\hline $\mathrm{C}(6)-\mathrm{C}(5)-\mathrm{H}(5 \mathrm{~B})$ & 110.4 \\
\hline $\mathrm{H}(5 \mathrm{~A})-\mathrm{C}(5)-\mathrm{H}(5 \mathrm{~B})$ & 108.6 \\
\hline $\mathrm{N}(2)-\mathrm{C}(6)-\mathrm{C}(5)$ & $102.08(11)$ \\
\hline $\mathrm{N}(2)-\mathrm{C}(6)-\mathrm{H}(6 \mathrm{~A})$ & 111.4 \\
\hline$C(5)-C(6)-H(6 A)$ & 111.4 \\
\hline $\mathrm{N}(2)-\mathrm{C}(6)-\mathrm{H}(6 \mathrm{~B})$ & 111.4 \\
\hline $\mathrm{C}(5)-\mathrm{C}(6)-\mathrm{H}(6 \mathrm{~B})$ & 111.4 \\
\hline
\end{tabular}




$\begin{array}{ll}\mathrm{H}(6 \mathrm{~A})-\mathrm{C}(6)-\mathrm{H}(6 \mathrm{~B}) & 109.2 \\ \mathrm{C}(4)-\mathrm{C}(7)-\mathrm{C}(8) & 130.52(13) \\ \mathrm{C}(4)-\mathrm{C}(7)-\mathrm{H}(7 \mathrm{~A}) & 114.7 \\ \mathrm{C}(8)-\mathrm{C}(7)-\mathrm{H}(7 \mathrm{~A}) & 114.7 \\ \mathrm{C}(9)-\mathrm{C}(8)-\mathrm{C}(13) & 116.98(13) \\ \mathrm{C}(9)-\mathrm{C}(8)-\mathrm{C}(7) & 116.36(13) \\ \mathrm{C}(13)-\mathrm{C}(8)-\mathrm{C}(7) & 126.65(13) \\ \mathrm{C}(10)-\mathrm{C}(9)-\mathrm{C}(8) & 122.15(13) \\ \mathrm{C}(10)-\mathrm{C}(9)-\mathrm{H}(9 \mathrm{~A}) & 118.9 \\ \mathrm{C}(8)-\mathrm{C}(9)-\mathrm{H}(9 \mathrm{~A}) & 118.9 \\ \mathrm{C}(9)-\mathrm{C}(10)-\mathrm{C}(11) & 119.97(14) \\ \mathrm{C}(9)-\mathrm{C}(10)-\mathrm{H}(10 \mathrm{~A}) & 120.0 \\ \mathrm{C}(11)-\mathrm{C}(10)-\mathrm{H}(10 \mathrm{~A}) & 120.0 \\ \mathrm{C}(12)-\mathrm{C}(11)-\mathrm{C}(10) & 119.05(15) \\ \mathrm{C}(12)-\mathrm{C}(11)-\mathrm{H}(11 \mathrm{~A}) & 120.5 \\ \mathrm{C}(10)-\mathrm{C}(11)-\mathrm{H}(11 \mathrm{~A}) & 120.5 \\ \mathrm{C}(11)-\mathrm{C}(12)-\mathrm{C}(13) & 121.13(14) \\ \mathrm{C}(11)-\mathrm{C}(12)-\mathrm{H}(12 \mathrm{~A}) & 119.4 \\ \mathrm{C}(13)-\mathrm{C}(12)-\mathrm{H}(12 \mathrm{~A}) & 119.4 \\ \mathrm{C}(12)-\mathrm{C}(13)-\mathrm{C}(8) & 120.71(13) \\ \mathrm{C}(12)-\mathrm{C}(13)-\mathrm{H}(13 \mathrm{~A}) & 119.6 \\ \mathrm{C}(8)-\mathrm{C}(13)-\mathrm{H}(13 \mathrm{~A}) & 119.6\end{array}$

Symmetry transformations used to generate equivalent atoms:

Table S1-4. Anisotropic displacement parameters $\left(\AA^{2} \times 1^{3}\right)$ for $17 \mathrm{~b}$. The anisotropic displacement factor exponent takes the form: $-2 p^{2}\left[h^{2} a^{* 2} U^{11}+\ldots+2 h k a^{*} b^{*} U^{12}\right]$

\begin{tabular}{lllllll}
\hline & $\mathrm{U}^{11}$ & $\mathrm{U}^{22}$ & $\mathrm{U}^{33}$ & $\mathrm{U}^{23}$ & $\mathrm{U}^{13}$ & $\mathrm{U}^{12}$ \\
\hline $\mathrm{O}(1)$ & $62(1)$ & $38(1)$ & $89(1)$ & $-3(1)$ & $-37(1)$ & $-18(1)$ \\
$\mathrm{O}(2)$ & $50(1)$ & $32(1)$ & $89(1)$ & $-2(1)$ & $-32(1)$ & $3(1)$ \\
$\mathrm{N}(1)$ & $31(1)$ & $42(1)$ & $40(1)$ & $6(1)$ & $-12(1)$ & $0(1)$ \\
$\mathrm{N}(2)$ & $28(1)$ & $29(1)$ & $33(1)$ & $3(1)$ & $-6(1)$ & $1(1)$ \\
$\mathrm{N}(3)$ & $40(1)$ & $31(1)$ & $49(1)$ & $-2(1)$ & $-12(1)$ & $-6(1)$
\end{tabular}




\begin{tabular}{lllllll}
$\mathrm{C}(1)$ & $33(1)$ & $39(1)$ & $37(1)$ & $3(1)$ & $-11(1)$ & $-7(1)$ \\
$\mathrm{C}(2)$ & $30(1)$ & $30(1)$ & $35(1)$ & $1(1)$ & $-7(1)$ & $-4(1)$ \\
$\mathrm{C}(3)$ & $27(1)$ & $28(1)$ & $28(1)$ & $2(1)$ & $-2(1)$ & $0(1)$ \\
$\mathrm{C}(4)$ & $26(1)$ & $27(1)$ & $26(1)$ & $-1(1)$ & $-1(1)$ & $-3(1)$ \\
$\mathrm{C}(5)$ & $37(1)$ & $26(1)$ & $34(1)$ & $1(1)$ & $-5(1)$ & $-2(1)$ \\
$\mathrm{C}(6)$ & $38(1)$ & $25(1)$ & $37(1)$ & $1(1)$ & $-2(1)$ & $2(1)$ \\
$\mathrm{C}(7)$ & $28(1)$ & $26(1)$ & $36(1)$ & $-1(1)$ & $-2(1)$ & $-3(1)$ \\
$\mathrm{C}(8)$ & $25(1)$ & $31(1)$ & $30(1)$ & $0(1)$ & $-1(1)$ & $-1(1)$ \\
$\mathrm{C}(9)$ & $34(1)$ & $28(1)$ & $53(1)$ & $0(1)$ & $-9(1)$ & $1(1)$ \\
$\mathrm{C}(10)$ & $34(1)$ & $38(1)$ & $59(1)$ & $4(1)$ & $-13(1)$ & $5(1)$ \\
$\mathrm{C}(11)$ & $27(1)$ & $47(1)$ & $39(1)$ & $1(1)$ & $-7(1)$ & $-2(1)$ \\
$\mathrm{C}(12)$ & $31(1)$ & $35(1)$ & $36(1)$ & $-1(1)$ & $-3(1)$ & $-8(1)$ \\
$\mathrm{C}(13)$ & $30(1)$ & $30(1)$ & $34(1)$ & $3(1)$ & $-2(1)$ & $-2(1)$ \\
\hline
\end{tabular}

Table S1-5. Hydrogen coordinates $\left(x \mathbf{1 0}^{4}\right)$ and isotropic displacement parameters $\left(\AA^{2} \mathbf{x}\right.$ $10^{3}$ ) for $17 b$.

\begin{tabular}{|c|c|c|c|c|}
\hline & $\mathrm{x}$ & $\mathrm{y}$ & $\mathrm{z}$ & $\mathrm{U}(\mathrm{eq})$ \\
\hline $\mathrm{H}(1 \mathrm{~A})$ & 4270 & 4109 & 4747 & 44 \\
\hline $\mathrm{H}(5 \mathrm{~A})$ & -1432 & 2080 & 1791 & 39 \\
\hline $\mathrm{H}(5 \mathrm{~B})$ & -2685 & 2007 & 2978 & 39 \\
\hline $\mathrm{H}(6 \mathrm{~A})$ & 172 & 1557 & 3905 & 40 \\
\hline $\mathrm{H}(6 \mathrm{~B})$ & 1440 & 1650 & 2719 & 40 \\
\hline $\mathrm{H}(7 \mathrm{~A})$ & -2592 & 4453 & 2452 & 36 \\
\hline $\mathrm{H}(9 \mathrm{~A})$ & -5318 & 5025 & 1482 & 46 \\
\hline $\mathrm{H}(10 \mathrm{~A})$ & -8328 & 4940 & 414 & 52 \\
\hline $\mathrm{H}(11 \mathrm{~A})$ & -9706 & 3560 & -79 & 45 \\
\hline $\mathrm{H}(12 \mathrm{~A})$ & -7961 & 2278 & 463 & 41 \\
\hline $\mathrm{H}(13 \mathrm{~A})$ & -4921 & 2354 & 1518 & 38 \\
\hline
\end{tabular}




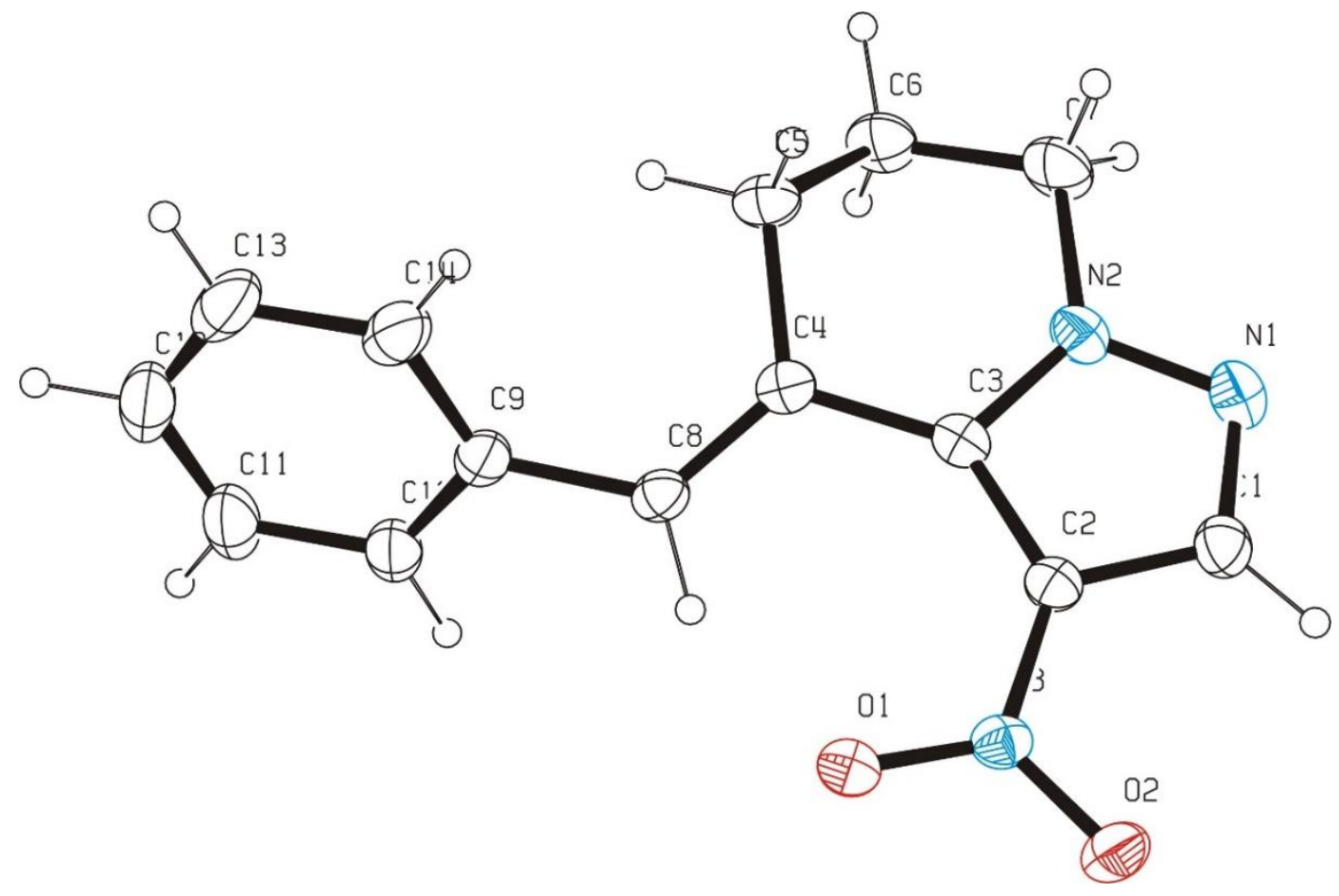

Figure S2. ORTEP diagram of 19b with anisotropic displacement parameters at $30 \%$ probability

Table S2-1. Crystal data and structure refinement for $\mathbf{1 9 b}$.

Identification code

Empirical formula

Formula weight

Temperature

Wavelength

Crystal system

Space group

Unit cell dimensions

Volume

Z

Density (calculated)

Absorption coefficient
$19 \mathrm{~b}$

C14 H13 N3 O2

255.27

173(2) K

$0.71073 \AA$

Triclinic

P-1

$\mathrm{a}=6.87710(10) \AA \quad \mathrm{a}=73.3380(10)^{\circ}$.

$\mathrm{b}=12.2722(2) \AA \quad \mathrm{b}=84.5950(10)^{\circ}$.

$\mathrm{c}=15.4408(3) \AA \quad \mathrm{g}=83.9060(10)^{\circ}$.

1238.61(4) $\AA^{3}$

4

$1.369 \mathrm{Mg} / \mathrm{m}^{3}$

$0.095 \mathrm{~mm}^{-1}$ 
$\mathrm{F}(000)$

Crystal size

Theta range for data collection

Index ranges

Reflections collected

Independent reflections

Completeness to theta $=26.00^{\circ}$

Absorption correction

Max. and min. transmission

Refinement method

Data / restraints / parameters

Goodness-of-fit on $\mathrm{F}^{2}$

Final R indices [I $>2 \operatorname{sigma}(\mathrm{I})]$

$\mathrm{R}$ indices (all data)

Largest diff. peak and hole
536

$0.60 \times 0.39 \times 0.38 \mathrm{~mm}^{3}$

1.74 to $26.00^{\circ}$.

$-8<=\mathrm{h}<=8,-15<=\mathrm{k}<=15,-18<=\mathrm{l}<=19$

20005

$4873[\mathrm{R}(\mathrm{int})=0.0213]$

$99.8 \%$

Semi-empirical from equivalents

0.9649 and 0.9454

Full-matrix least-squares on $\mathrm{F}^{2}$

4873 / 0 / 343

1.055

$\mathrm{R} 1=0.0431, \mathrm{wR} 2=0.1096$

$\mathrm{R} 1=0.0482, \mathrm{wR} 2=0.1144$

0.204 and -0.326 e. $\AA^{-3}$

Table S2-2. Atomic coordinates ( $x 1^{4}$ ) and equivalent isotropic displacement parameters $\left(\AA^{2} \times 10^{3}\right)$ for $19 \mathrm{~b}$. $U(\mathrm{eq})$ is defined as one third of the trace of the orthogonalized $\mathrm{U}^{\mathrm{ij}}$ tensor.

\begin{tabular}{lcccc}
\hline & $\mathrm{x}$ & $\mathrm{y}$ & $\mathrm{z}$ & $\mathrm{U}(\mathrm{eq})$ \\
\hline $\mathrm{O}(1)$ & $6981(1)$ & $3587(1)$ & $1620(1)$ & $41(1)$ \\
$\mathrm{O}(2)$ & $6700(2)$ & $5317(1)$ & $1724(1)$ & $51(1)$ \\
$\mathrm{O}(3)$ & $2069(2)$ & $2269(1)$ & $3805(1)$ & $57(1)$ \\
$\mathrm{O}(4)$ & $2166(2)$ & $3051(1)$ & $4875(1)$ & $76(1)$ \\
$\mathrm{N}(1)$ & $7025(2)$ & $4074(1)$ & $4417(1)$ & $48(1)$ \\
$\mathrm{N}(2)$ & $7102(2)$ & $2953(1)$ & $4441(1)$ & $40(1)$ \\
$\mathrm{N}(3)$ & $6841(2)$ & $4271(1)$ & $2073(1)$ & $34(1)$ \\
$\mathrm{N}(4)$ & $1725(2)$ & $6138(1)$ & $3071(1)$ & $51(1)$ \\
$\mathrm{N}(5)$ & $1738(2)$ & $5756(1)$ & $2325(1)$ & $40(1)$ \\
$\mathrm{N}(6)$ & $2037(2)$ & $3119(1)$ & $4073(1)$ & $42(1)$ \\
$\mathrm{C}(1)$ & $6873(2)$ & $4633(1)$ & $3561(1)$ & $41(1)$ \\
$\mathrm{C}(2)$ & $6862(2)$ & $3882(1)$ & $3030(1)$ & $33(1)$ \\
$\mathrm{C}(3)$ & $7008(2)$ & $2771(1)$ & $3623(1)$ & $32(1)$
\end{tabular}




\begin{tabular}{lrrrr}
$\mathrm{C}(4)$ & $7115(2)$ & $1591(1)$ & $3564(1)$ & $33(1)$ \\
$\mathrm{C}(5)$ & $7932(2)$ & $728(1)$ & $4383(1)$ & $42(1)$ \\
$\mathrm{C}(6)$ & $6903(2)$ & $951(1)$ & $5242(1)$ & $49(1)$ \\
$\mathrm{C}(7)$ & $7329(3)$ & $2099(1)$ & $5317(1)$ & $54(1)$ \\
$\mathrm{C}(8)$ & $6365(2)$ & $1323(1)$ & $2889(1)$ & $35(1)$ \\
$\mathrm{C}(9)$ & $6375(2)$ & $181(1)$ & $2753(1)$ & $40(1)$ \\
$\mathrm{C}(10)$ & $4724(3)$ & $-96(1)$ & $2429(1)$ & $49(1)$ \\
$\mathrm{C}(11)$ & $4631(4)$ & $-1152(1)$ & $2291(1)$ & $68(1)$ \\
$\mathrm{C}(12)$ & $6193(4)$ & $-1950(2)$ & $2459(1)$ & $81(1)$ \\
$\mathrm{C}(13)$ & $7861(4)$ & $-1698(2)$ & $2765(1)$ & $78(1)$ \\
$\mathrm{C}(14)$ & $7978(3)$ & $-632(1)$ & $2908(1)$ & $56(1)$ \\
$\mathrm{C}(15)$ & $1802(2)$ & $5206(1)$ & $3754(1)$ & $47(1)$ \\
$\mathrm{C}(16)$ & $1871(2)$ & $4234(1)$ & $3457(1)$ & $36(1)$ \\
$\mathrm{C}(17)$ & $1840(2)$ & $4607(1)$ & $2506(1)$ & $32(1)$ \\
$\mathrm{C}(18)$ & $2010(2)$ & $4099(1)$ & $1745(1)$ & $32(1)$ \\
$\mathrm{C}(19)$ & $2519(2)$ & $4918(1)$ & $830(1)$ & $38(1)$ \\
$\mathrm{C}(20)$ & $1328(2)$ & $6059(1)$ & $725(1)$ & $46(1)$ \\
$\mathrm{C}(21)$ & $1796(3)$ & $6592(1)$ & $1437(1)$ & $54(1)$ \\
$\mathrm{C}(22)$ & $1628(2)$ & $3021(1)$ & $1855(1)$ & $35(1)$ \\
$\mathrm{C}(23)$ & $1912(2)$ & $2382(1)$ & $1176(1)$ & $37(1)$ \\
$\mathrm{C}(24)$ & $3554(2)$ & $2445(1)$ & $567(1)$ & $47(1)$ \\
$\mathrm{C}(25)$ & $3839(3)$ & $1751(2)$ & $-3(1)$ & $64(1)$ \\
$\mathrm{C}(26)$ & $2508(3)$ & $984(2)$ & $16(1)$ & $73(1)$ \\
$\mathrm{C}(27)$ & $580(3)$ & $905(2)$ & $622(1)$ & $67(1)$ \\
$\mathrm{C}(28)$ & $587(2)$ & $1591(1)$ & $1195(1)$ & $49(1)$ \\
\hline & & & &
\end{tabular}

Table S2-3. Bond lengths $[\AA ̊ \AA]$ and angles $\left[{ }^{\circ}\right]$ for $19 b$.

\begin{tabular}{ll}
\hline $\mathrm{O}(1)-\mathrm{N}(3)$ & $1.2282(14)$ \\
$\mathrm{O}(2)-\mathrm{N}(3)$ & $1.2385(14)$ \\
$\mathrm{O}(3)-\mathrm{N}(6)$ & $1.2253(17)$ \\
$\mathrm{O}(4)-\mathrm{N}(6)$ & $1.2276(16)$ \\
$\mathrm{N}(1)-\mathrm{C}(1)$ & $1.312(2)$ \\
$\mathrm{N}(1)-\mathrm{N}(2)$ & $1.3599(17)$ \\
$\mathrm{N}(2)-\mathrm{C}(3)$ & $1.3542(17)$
\end{tabular}




\begin{tabular}{|c|c|}
\hline $\mathrm{N}(2)-\mathrm{C}(7)$ & $1.4636(19)$ \\
\hline $\mathrm{N}(3)-\mathrm{C}(2)$ & $1.4188(17)$ \\
\hline $\mathrm{N}(4)-\mathrm{C}(15)$ & $1.315(2)$ \\
\hline $\mathrm{N}(4)-\mathrm{N}(5)$ & $1.3618(17)$ \\
\hline $\mathrm{N}(5)-\mathrm{C}(17)$ & $1.3517(17)$ \\
\hline $\mathrm{N}(5)-\mathrm{C}(21)$ & $1.4581(19)$ \\
\hline $\mathrm{N}(6)-\mathrm{C}(16)$ & $1.4254(19)$ \\
\hline$C(1)-C(2)$ & $1.3973(19)$ \\
\hline $\mathrm{C}(1)-\mathrm{H}(1 \mathrm{~A})$ & 0.9500 \\
\hline$C(2)-C(3)$ & $1.4073(18)$ \\
\hline$C(3)-C(4)$ & $1.4705(18)$ \\
\hline $\mathrm{C}(4)-\mathrm{C}(8)$ & $1.3396(19)$ \\
\hline$C(4)-C(5)$ & $1.5089(18)$ \\
\hline$C(5)-C(6)$ & $1.525(2)$ \\
\hline $\mathrm{C}(5)-\mathrm{H}(5 \mathrm{~A})$ & 0.9900 \\
\hline $\mathrm{C}(5)-\mathrm{H}(5 \mathrm{~B})$ & 0.9900 \\
\hline$C(6)-C(7)$ & $1.508(2)$ \\
\hline $\mathrm{C}(6)-\mathrm{H}(6 \mathrm{~A})$ & 0.9900 \\
\hline $\mathrm{C}(6)-\mathrm{H}(6 \mathrm{~B})$ & 0.9900 \\
\hline $\mathrm{C}(7)-\mathrm{H}(7 \mathrm{~A})$ & 0.9900 \\
\hline $\mathrm{C}(7)-\mathrm{H}(7 \mathrm{~B})$ & 0.9900 \\
\hline $\mathrm{C}(8)-\mathrm{C}(9)$ & $1.4733(18)$ \\
\hline $\mathrm{C}(8)-\mathrm{H}(8 \mathrm{~A})$ & 0.9500 \\
\hline $\mathrm{C}(9)-\mathrm{C}(10)$ & $1.394(2)$ \\
\hline $\mathrm{C}(9)-\mathrm{C}(14)$ & $1.396(2)$ \\
\hline $\mathrm{C}(10)-\mathrm{C}(11)$ & $1.381(2)$ \\
\hline $\mathrm{C}(10)-\mathrm{H}(10 \mathrm{~A})$ & 0.9500 \\
\hline $\mathrm{C}(11)-\mathrm{C}(12)$ & $1.365(3)$ \\
\hline $\mathrm{C}(11)-\mathrm{H}(11 \mathrm{~A})$ & 0.9500 \\
\hline $\mathrm{C}(12)-\mathrm{C}(13)$ & $1.377(3)$ \\
\hline $\mathrm{C}(12)-\mathrm{H}(12 \mathrm{~A})$ & 0.9500 \\
\hline $\mathrm{C}(13)-\mathrm{C}(14)$ & $1.399(3)$ \\
\hline $\mathrm{C}(13)-\mathrm{H}(13 \mathrm{~A})$ & 0.9500 \\
\hline $\mathrm{C}(14)-\mathrm{H}(14 \mathrm{~A})$ & 0.9500 \\
\hline $\mathrm{C}(15)-\mathrm{C}(16)$ & $1.391(2)$ \\
\hline $\mathrm{C}(15)-\mathrm{H}(15 \mathrm{~A})$ & 0.9500 \\
\hline
\end{tabular}




\begin{tabular}{|c|c|}
\hline$C(16)-C(17)$ & $1.4076(18)$ \\
\hline $\mathrm{C}(17)-\mathrm{C}(18)$ & $1.4719(17)$ \\
\hline$C(18)-C(22)$ & $1.3358(18)$ \\
\hline $\mathrm{C}(18)-\mathrm{C}(19)$ & $1.5152(18)$ \\
\hline $\mathrm{C}(19)-\mathrm{C}(20)$ & $1.519(2)$ \\
\hline C(19)-H(19A) & 0.9900 \\
\hline C(19)-H(19B) & 0.9900 \\
\hline$C(20)-C(21)$ & $1.504(2)$ \\
\hline $\mathrm{C}(20)-\mathrm{H}(20 \mathrm{~A})$ & 0.9900 \\
\hline $\mathrm{C}(20)-\mathrm{H}(20 \mathrm{~B})$ & 0.9900 \\
\hline $\mathrm{C}(21)-\mathrm{H}(21 \mathrm{~A})$ & 0.9900 \\
\hline $\mathrm{C}(21)-\mathrm{H}(21 \mathrm{~B})$ & 0.9900 \\
\hline $\mathrm{C}(22)-\mathrm{C}(23)$ & $1.4676(18)$ \\
\hline $\mathrm{C}(22)-\mathrm{H}(22 \mathrm{~A})$ & 0.9500 \\
\hline $\mathrm{C}(23)-\mathrm{C}(28)$ & $1.3926(19)$ \\
\hline $\mathrm{C}(23)-\mathrm{C}(24)$ & $1.394(2)$ \\
\hline $\mathrm{C}(24)-\mathrm{C}(25)$ & $1.378(2)$ \\
\hline $\mathrm{C}(24)-\mathrm{H}(24 \mathrm{~A})$ & 0.9500 \\
\hline$C(25)-C(26)$ & $1.373(2)$ \\
\hline $\mathrm{C}(25)-\mathrm{H}(25 \mathrm{~A})$ & 0.9500 \\
\hline$C(26)-C(27)$ & $1.382(3)$ \\
\hline $\mathrm{C}(26)-\mathrm{H}(26 \mathrm{~A})$ & 0.9500 \\
\hline $\mathrm{C}(27)-\mathrm{C}(28)$ & $1.375(2)$ \\
\hline $\mathrm{C}(27)-\mathrm{H}(27 \mathrm{~A})$ & 0.9500 \\
\hline $\mathrm{C}(28)-\mathrm{H}(28 \mathrm{~A})$ & 0.9500 \\
\hline $\mathrm{C}(1)-\mathrm{N}(1)-\mathrm{N}(2)$ & $104.86(11)$ \\
\hline $\mathrm{C}(3)-\mathrm{N}(2)-\mathrm{N}(1)$ & $114.19(11)$ \\
\hline $\mathrm{C}(3)-\mathrm{N}(2)-\mathrm{C}(7)$ & $127.77(12)$ \\
\hline $\mathrm{N}(1)-\mathrm{N}(2)-\mathrm{C}(7)$ & $118.02(12)$ \\
\hline $\mathrm{O}(1)-\mathrm{N}(3)-\mathrm{O}(2)$ & $122.41(12)$ \\
\hline $\mathrm{O}(1)-\mathrm{N}(3)-\mathrm{C}(2)$ & $120.54(11)$ \\
\hline $\mathrm{O}(2)-\mathrm{N}(3)-\mathrm{C}(2)$ & $117.05(11)$ \\
\hline $\mathrm{C}(15)-\mathrm{N}(4)-\mathrm{N}(5)$ & $104.62(12)$ \\
\hline $\mathrm{C}(17)-\mathrm{N}(5)-\mathrm{N}(4)$ & $114.19(12)$ \\
\hline $\mathrm{C}(17)-\mathrm{N}(5)-\mathrm{C}(21)$ & $127.41(12)$ \\
\hline
\end{tabular}




\begin{tabular}{|c|c|}
\hline $\mathrm{N}(4)-\mathrm{N}(5)-\mathrm{C}(21)$ & $118.22(12)$ \\
\hline $\mathrm{O}(3)-\mathrm{N}(6)-\mathrm{O}(4)$ & $121.89(13)$ \\
\hline $\mathrm{O}(3)-\mathrm{N}(6)-\mathrm{C}(16)$ & $120.86(12)$ \\
\hline $\mathrm{O}(4)-\mathrm{N}(6)-\mathrm{C}(16)$ & $117.25(13)$ \\
\hline $\mathrm{N}(1)-\mathrm{C}(1)-\mathrm{C}(2)$ & $111.06(13)$ \\
\hline $\mathrm{N}(1)-\mathrm{C}(1)-\mathrm{H}(1 \mathrm{~A})$ & 124.5 \\
\hline $\mathrm{C}(2)-\mathrm{C}(1)-\mathrm{H}(1 \mathrm{~A})$ & 124.5 \\
\hline$C(1)-C(2)-C(3)$ & $106.80(12)$ \\
\hline $\mathrm{C}(1)-\mathrm{C}(2)-\mathrm{N}(3)$ & $122.08(12)$ \\
\hline $\mathrm{C}(3)-\mathrm{C}(2)-\mathrm{N}(3)$ & $130.98(12)$ \\
\hline $\mathrm{N}(2)-\mathrm{C}(3)-\mathrm{C}(2)$ & $103.09(11)$ \\
\hline $\mathrm{N}(2)-\mathrm{C}(3)-\mathrm{C}(4)$ & $119.02(11)$ \\
\hline$C(2)-C(3)-C(4)$ & $137.88(12)$ \\
\hline $\mathrm{C}(8)-\mathrm{C}(4)-\mathrm{C}(3)$ & $122.17(11)$ \\
\hline $\mathrm{C}(8)-\mathrm{C}(4)-\mathrm{C}(5)$ & $124.37(12)$ \\
\hline$C(3)-C(4)-C(5)$ & $113.11(11)$ \\
\hline$C(4)-C(5)-C(6)$ & $109.56(12)$ \\
\hline $\mathrm{C}(4)-\mathrm{C}(5)-\mathrm{H}(5 \mathrm{~A})$ & 109.8 \\
\hline $\mathrm{C}(6)-\mathrm{C}(5)-\mathrm{H}(5 \mathrm{~A})$ & 109.8 \\
\hline $\mathrm{C}(4)-\mathrm{C}(5)-\mathrm{H}(5 \mathrm{~B})$ & 109.8 \\
\hline $\mathrm{C}(6)-\mathrm{C}(5)-\mathrm{H}(5 \mathrm{~B})$ & 109.8 \\
\hline $\mathrm{H}(5 \mathrm{~A})-\mathrm{C}(5)-\mathrm{H}(5 \mathrm{~B})$ & 108.2 \\
\hline$C(7)-C(6)-C(5)$ & $110.54(12)$ \\
\hline $\mathrm{C}(7)-\mathrm{C}(6)-\mathrm{H}(6 \mathrm{~A})$ & 109.5 \\
\hline $\mathrm{C}(5)-\mathrm{C}(6)-\mathrm{H}(6 \mathrm{~A})$ & 109.5 \\
\hline $\mathrm{C}(7)-\mathrm{C}(6)-\mathrm{H}(6 \mathrm{~B})$ & 109.5 \\
\hline $\mathrm{C}(5)-\mathrm{C}(6)-\mathrm{H}(6 \mathrm{~B})$ & 109.5 \\
\hline $\mathrm{H}(6 \mathrm{~A})-\mathrm{C}(6)-\mathrm{H}(6 \mathrm{~B})$ & 108.1 \\
\hline$N(2)-C(7)-C(6)$ & $109.47(12)$ \\
\hline $\mathrm{N}(2)-\mathrm{C}(7)-\mathrm{H}(7 \mathrm{~A})$ & 109.8 \\
\hline $\mathrm{C}(6)-\mathrm{C}(7)-\mathrm{H}(7 \mathrm{~A})$ & 109.8 \\
\hline $\mathrm{N}(2)-\mathrm{C}(7)-\mathrm{H}(7 \mathrm{~B})$ & 109.8 \\
\hline $\mathrm{C}(6)-\mathrm{C}(7)-\mathrm{H}(7 \mathrm{~B})$ & 109.8 \\
\hline $\mathrm{H}(7 \mathrm{~A})-\mathrm{C}(7)-\mathrm{H}(7 \mathrm{~B})$ & 108.2 \\
\hline$C(4)-C(8)-C(9)$ & $127.42(12)$ \\
\hline $\mathrm{C}(4)-\mathrm{C}(8)-\mathrm{H}(8 \mathrm{~A})$ & 116.3 \\
\hline
\end{tabular}




\begin{tabular}{|c|c|}
\hline $\mathrm{C}(9)-\mathrm{C}(8)-\mathrm{H}(8 \mathrm{~A})$ & 116.3 \\
\hline$C(10)-C(9)-C(14)$ & $117.84(14)$ \\
\hline $\mathrm{C}(10)-\mathrm{C}(9)-\mathrm{C}(8)$ & $118.67(13)$ \\
\hline $\mathrm{C}(14)-\mathrm{C}(9)-\mathrm{C}(8)$ & $123.46(15)$ \\
\hline $\mathrm{C}(11)-\mathrm{C}(10)-\mathrm{C}(9)$ & $121.66(17)$ \\
\hline $\mathrm{C}(11)-\mathrm{C}(10)-\mathrm{H}(10 \mathrm{~A})$ & 119.2 \\
\hline $\mathrm{C}(9)-\mathrm{C}(10)-\mathrm{H}(10 \mathrm{~A})$ & 119.2 \\
\hline$C(12)-C(11)-C(10)$ & $120.1(2)$ \\
\hline $\mathrm{C}(12)-\mathrm{C}(11)-\mathrm{H}(11 \mathrm{~A})$ & 120.0 \\
\hline $\mathrm{C}(10)-\mathrm{C}(11)-\mathrm{H}(11 \mathrm{~A})$ & 120.0 \\
\hline$C(11)-C(12)-C(13)$ & $119.82(17)$ \\
\hline $\mathrm{C}(11)-\mathrm{C}(12)-\mathrm{H}(12 \mathrm{~A})$ & 120.1 \\
\hline $\mathrm{C}(13)-\mathrm{C}(12)-\mathrm{H}(12 \mathrm{~A})$ & 120.1 \\
\hline$C(12)-C(13)-C(14)$ & $120.82(18)$ \\
\hline $\mathrm{C}(12)-\mathrm{C}(13)-\mathrm{H}(13 \mathrm{~A})$ & 119.6 \\
\hline $\mathrm{C}(14)-\mathrm{C}(13)-\mathrm{H}(13 \mathrm{~A})$ & 119.6 \\
\hline $\mathrm{C}(9)-\mathrm{C}(14)-\mathrm{C}(13)$ & $119.75(19)$ \\
\hline $\mathrm{C}(9)-\mathrm{C}(14)-\mathrm{H}(14 \mathrm{~A})$ & 120.1 \\
\hline $\mathrm{C}(13)-\mathrm{C}(14)-\mathrm{H}(14 \mathrm{~A})$ & 120.1 \\
\hline $\mathrm{N}(4)-\mathrm{C}(15)-\mathrm{C}(16)$ & $111.19(13)$ \\
\hline $\mathrm{N}(4)-\mathrm{C}(15)-\mathrm{H}(15 \mathrm{~A})$ & 124.4 \\
\hline $\mathrm{C}(16)-\mathrm{C}(15)-\mathrm{H}(15 \mathrm{~A})$ & 124.4 \\
\hline$C(15)-C(16)-C(17)$ & $106.87(13)$ \\
\hline$C(15)-C(16)-N(6)$ & $121.40(13)$ \\
\hline $\mathrm{C}(17)-\mathrm{C}(16)-\mathrm{N}(6)$ & $131.66(12)$ \\
\hline $\mathrm{N}(5)-\mathrm{C}(17)-\mathrm{C}(16)$ & $103.13(11)$ \\
\hline $\mathrm{N}(5)-\mathrm{C}(17)-\mathrm{C}(18)$ & $118.73(11)$ \\
\hline$C(16)-C(17)-C(18)$ & $137.98(12)$ \\
\hline $\mathrm{C}(22)-\mathrm{C}(18)-\mathrm{C}(17)$ & $121.99(12)$ \\
\hline $\mathrm{C}(22)-\mathrm{C}(18)-\mathrm{C}(19)$ & $123.45(12)$ \\
\hline $\mathrm{C}(17)-\mathrm{C}(18)-\mathrm{C}(19)$ & $114.44(11)$ \\
\hline$C(18)-C(19)-C(20)$ & $110.47(11)$ \\
\hline $\mathrm{C}(18)-\mathrm{C}(19)-\mathrm{H}(19 \mathrm{~A})$ & 109.6 \\
\hline $\mathrm{C}(20)-\mathrm{C}(19)-\mathrm{H}(19 \mathrm{~A})$ & 109.6 \\
\hline $\mathrm{C}(18)-\mathrm{C}(19)-\mathrm{H}(19 \mathrm{~B})$ & 109.6 \\
\hline $\mathrm{C}(20)-\mathrm{C}(19)-\mathrm{H}(19 \mathrm{~B})$ & 109.6 \\
\hline
\end{tabular}




$\begin{array}{ll}\mathrm{H}(19 \mathrm{~A})-\mathrm{C}(19)-\mathrm{H}(19 \mathrm{~B}) & 108.1 \\ \mathrm{C}(21)-\mathrm{C}(20)-\mathrm{C}(19) & 110.09(12) \\ \mathrm{C}(21)-\mathrm{C}(20)-\mathrm{H}(20 \mathrm{~A}) & 109.6 \\ \mathrm{C}(19)-\mathrm{C}(20)-\mathrm{H}(20 \mathrm{~A}) & 109.6 \\ \mathrm{C}(21)-\mathrm{C}(20)-\mathrm{H}(20 \mathrm{~B}) & 109.6 \\ \mathrm{C}(19)-\mathrm{C}(20)-\mathrm{H}(20 \mathrm{~B}) & 109.6 \\ \mathrm{H}(20 \mathrm{~A})-\mathrm{C}(20)-\mathrm{H}(20 \mathrm{~B}) & 108.2 \\ \mathrm{~N}(5)-\mathrm{C}(21)-\mathrm{C}(20) & 110.18(12) \\ \mathrm{N}(5)-\mathrm{C}(21)-\mathrm{H}(21 \mathrm{~A}) & 109.6 \\ \mathrm{C}(20)-\mathrm{C}(21)-\mathrm{H}(21 \mathrm{~A}) & 109.6 \\ \mathrm{~N}(5)-\mathrm{C}(21)-\mathrm{H}(21 \mathrm{~B}) & 109.6 \\ \mathrm{C}(20)-\mathrm{C}(21)-\mathrm{H}(21 \mathrm{~B}) & 109.6 \\ \mathrm{H}(21 \mathrm{~A})-\mathrm{C}(21)-\mathrm{H}(21 \mathrm{~B}) & 108.1 \\ \mathrm{C}(18)-\mathrm{C}(22)-\mathrm{C}(23) & 127.39(12) \\ \mathrm{C}(18)-\mathrm{C}(22)-\mathrm{H}(22 \mathrm{~A}) & 116.3 \\ \mathrm{C}(23)-\mathrm{C}(22)-\mathrm{H}(22 \mathrm{~A}) & 116.3 \\ \mathrm{C}(28)-\mathrm{C}(23)-\mathrm{C}(24) & 117.96(13) \\ \mathrm{C}(28)-\mathrm{C}(23)-\mathrm{C}(22) & 118.80(12) \\ \mathrm{C}(24)-\mathrm{C}(23)-\mathrm{C}(22) & 122.94(12) \\ \mathrm{C}(25)-\mathrm{C}(24)-\mathrm{C}(23) & 120.69(14) \\ \mathrm{C}(25)-\mathrm{C}(24)-\mathrm{H}(24 \mathrm{~A}) & 119.7 \\ \mathrm{C}(23)-\mathrm{C}(24)-\mathrm{H}(24 \mathrm{~A}) & 119.7 \\ \mathrm{C}(26)-\mathrm{C}(25)-\mathrm{C}(24) & 120.75(16) \\ \mathrm{C}(26)-\mathrm{C}(25)-\mathrm{H}(25 \mathrm{~A}) & 119.6 \\ \mathrm{C}(24)-\mathrm{C}(25)-\mathrm{H}(25 \mathrm{~A}) & 119.6 \\ \mathrm{C}(25)-\mathrm{C}(26)-\mathrm{C}(27) & 119.21(16) \\ \mathrm{C}(25)-\mathrm{C}(26)-\mathrm{H}(26 \mathrm{~A}) & 120.4 \\ \mathrm{C}(27)-\mathrm{C}(26)-\mathrm{H}(26 \mathrm{~A}) & 120.4 \\ \mathrm{C}(28)-\mathrm{C}(27)-\mathrm{C}(26) & 120.47(15) \\ \mathrm{C}(28)-\mathrm{C}(27)-\mathrm{H}(27 \mathrm{~A}) & 119.8 \\ \mathrm{C}(26)-\mathrm{C}(27)-\mathrm{H}(27 \mathrm{~A}) & 119.8 \\ \mathrm{C}(27)-\mathrm{C}(28)-\mathrm{C}(23) & 120.91(14) \\ \mathrm{C}(27)-\mathrm{C}(28)-\mathrm{H}(28 \mathrm{~A}) & 119.5 \\ \mathrm{C}(23)-\mathrm{C}(28)-\mathrm{H}(28 \mathrm{~A}) & 119.5 \\ & \end{array}$

Symmetry transformations used to generate equivalent atoms: 
Table S2-4. Anisotropic displacement parameters $\left(\AA^{2} \times 1^{3}\right)$ for $19 b$. The anisotropic displacement factor exponent takes the form: $-2 p^{2}\left[h^{2} a^{* 2} U^{11}+\ldots+2 h k a^{*} b^{*} U^{12}\right]$

\begin{tabular}{|c|c|c|c|c|c|c|}
\hline & $\mathrm{U}^{11}$ & $\mathrm{U}^{22}$ & $\mathrm{U}^{33}$ & $\mathrm{U}^{23}$ & $\mathrm{U}^{13}$ & $\mathrm{U}^{12}$ \\
\hline $\mathrm{O}(1)$ & $48(1)$ & $43(1)$ & $33(1)$ & $-9(1)$ & $-5(1)$ & $-3(1)$ \\
\hline $\mathrm{O}(2)$ & $60(1)$ & $35(1)$ & $51(1)$ & $0(1)$ & $-12(1)$ & $-5(1)$ \\
\hline $\mathrm{O}(3)$ & $77(1)$ & $51(1)$ & $41(1)$ & $-7(1)$ & $-10(1)$ & $-4(1)$ \\
\hline $\mathrm{O}(4)$ & $110(1)$ & $88(1)$ & $28(1)$ & $-9(1)$ & $-15(1)$ & $-11(1)$ \\
\hline $\mathrm{N}(1)$ & $54(1)$ & $51(1)$ & $46(1)$ & $-23(1)$ & $-5(1)$ & $-8(1)$ \\
\hline $\mathrm{N}(2)$ & $43(1)$ & $48(1)$ & $32(1)$ & $-14(1)$ & $-2(1)$ & $-7(1)$ \\
\hline $\mathrm{N}(3)$ & $26(1)$ & $37(1)$ & $38(1)$ & $-6(1)$ & $-6(1)$ & $-3(1)$ \\
\hline $\mathrm{N}(4)$ & $59(1)$ & $56(1)$ & $48(1)$ & $-29(1)$ & $1(1)$ & $-7(1)$ \\
\hline $\mathrm{N}(5)$ & $42(1)$ & $44(1)$ & $37(1)$ & $-16(1)$ & $0(1)$ & $-6(1)$ \\
\hline $\mathrm{N}(6)$ & $36(1)$ & $59(1)$ & $30(1)$ & $-9(1)$ & $-4(1)$ & $-5(1)$ \\
\hline $\mathrm{C}(1)$ & $39(1)$ & $41(1)$ & $48(1)$ & $-16(1)$ & $-7(1)$ & $-4(1)$ \\
\hline$C(2)$ & $25(1)$ & $38(1)$ & $36(1)$ & $-10(1)$ & $-4(1)$ & $-1(1)$ \\
\hline $\mathrm{C}(3)$ & $25(1)$ & $42(1)$ & $30(1)$ & $-11(1)$ & $0(1)$ & $-3(1)$ \\
\hline $\mathrm{C}(4)$ & $29(1)$ & $36(1)$ & $30(1)$ & $-6(1)$ & $3(1)$ & $0(1)$ \\
\hline$C(5)$ & $43(1)$ & $45(1)$ & $32(1)$ & $-3(1)$ & $-2(1)$ & $1(1)$ \\
\hline$C(6)$ & $57(1)$ & $54(1)$ & $29(1)$ & $-3(1)$ & $4(1)$ & $-8(1)$ \\
\hline$C(7)$ & $72(1)$ & $63(1)$ & $27(1)$ & $-10(1)$ & $-2(1)$ & $-10(1)$ \\
\hline $\mathrm{C}(8)$ & $37(1)$ & $33(1)$ & $30(1)$ & $-6(1)$ & $1(1)$ & $1(1)$ \\
\hline $\mathrm{C}(9)$ & $57(1)$ & $33(1)$ & $25(1)$ & $-5(1)$ & $6(1)$ & $-1(1)$ \\
\hline$C(10)$ & $72(1)$ & $39(1)$ & $36(1)$ & $-10(1)$ & $1(1)$ & $-11(1)$ \\
\hline $\mathrm{C}(11)$ & $120(2)$ & $44(1)$ & $43(1)$ & $-14(1)$ & $2(1)$ & $-24(1)$ \\
\hline $\mathrm{C}(12)$ & $164(2)$ & $36(1)$ & $41(1)$ & $-13(1)$ & $7(1)$ & $-7(1)$ \\
\hline $\mathrm{C}(13)$ & $132(2)$ & $45(1)$ & $42(1)$ & $-8(1)$ & $9(1)$ & $33(1)$ \\
\hline $\mathrm{C}(14)$ & $75(1)$ & $47(1)$ & $38(1)$ & $-9(1)$ & $4(1)$ & $17(1)$ \\
\hline$C(15)$ & $45(1)$ & $65(1)$ & $38(1)$ & $-25(1)$ & $-1(1)$ & $-7(1)$ \\
\hline$C(16)$ & $26(1)$ & $52(1)$ & $31(1)$ & $-15(1)$ & $-1(1)$ & $-4(1)$ \\
\hline $\mathrm{C}(17)$ & $23(1)$ & $42(1)$ & $33(1)$ & $-12(1)$ & $-1(1)$ & $-5(1)$ \\
\hline $\mathrm{C}(18)$ & $24(1)$ & $44(1)$ & $27(1)$ & $-11(1)$ & $-3(1)$ & $-4(1)$ \\
\hline C(19) & $41(1)$ & $47(1)$ & $28(1)$ & $-9(1)$ & $-3(1)$ & $-9(1)$ \\
\hline $\mathrm{C}(20)$ & $48(1)$ & $50(1)$ & $36(1)$ & $-2(1)$ & $-5(1)$ & $-3(1)$ \\
\hline $\mathrm{C}(21)$ & $71(1)$ & $41(1)$ & $45(1)$ & $-8(1)$ & $3(1)$ & $-6(1)$ \\
\hline
\end{tabular}




$\begin{array}{lllllll}\mathrm{C}(22) & 32(1) & 44(1) & 29(1) & -10(1) & 1(1) & -6(1) \\ \mathrm{C}(23) & 40(1) & 42(1) & 30(1) & -12(1) & -1(1) & -8(1) \\ \mathrm{C}(24) & 52(1) & 53(1) & 42(1) & -20(1) & 10(1) & -18(1) \\ \mathrm{C}(25) & 76(1) & 71(1) & 54(1) & -33(1) & 28(1) & -29(1) \\ \mathrm{C}(26) & 93(1) & 82(1) & 61(1) & -48(1) & 23(1) & -36(1) \\ \mathrm{C}(27) & 73(1) & 80(1) & 67(1) & -44(1) & 13(1) & -38(1) \\ \mathrm{C}(28) & 47(1) & 59(1) & 46(1) & -22(1) & 7(1) & -19(1)\end{array}$

Table S2-5. Hydrogen coordinates ( $\left.x \mathbf{1 0}^{4}\right)$ and isotropic displacement parameters $\left(\AA^{2} \mathbf{x}\right.$ 103 ) for $19 b$.

\begin{tabular}{lrrrl}
\hline & $\mathrm{x}$ & $\mathrm{y}$ & $\mathrm{z}$ & $\mathrm{U}(\mathrm{eq})$ \\
\hline $\mathrm{H}(1 \mathrm{~A})$ & 6783 & 5441 & 3331 & 50 \\
$\mathrm{H}(5 \mathrm{~A})$ & 9358 & 786 & 4379 & 50 \\
$\mathrm{H}(5 \mathrm{~B})$ & 7729 & -52 & 4367 & 50 \\
$\mathrm{H}(6 \mathrm{~A})$ & 5471 & 927 & 5231 & 58 \\
$\mathrm{H}(6 \mathrm{~B})$ & 7357 & 346 & 5778 & 58 \\
$\mathrm{H}(7 \mathrm{~A})$ & 6415 & 2318 & 5785 & 65 \\
$\mathrm{H}(7 \mathrm{~B})$ & 8683 & 2060 & 5499 & 65 \\
$\mathrm{H}(8 \mathrm{~A})$ & 5757 & 1939 & 2447 & 42 \\
$\mathrm{H}(10 \mathrm{~A})$ & 3635 & 456 & 2299 & 58 \\
$\mathrm{H}(11 \mathrm{~A})$ & 3479 & -1324 & 2080 & 81 \\
$\mathrm{H}(12 \mathrm{~A})$ & 6130 & -2676 & 2364 & 97 \\
$\mathrm{H}(13 \mathrm{~A})$ & 8945 & -2255 & 2881 & 93 \\
$\mathrm{H}(14 \mathrm{~A})$ & 9144 & -463 & 3109 & 67 \\
$\mathrm{H}(15 \mathrm{~A})$ & 1811 & 5198 & 4371 & 56 \\
$\mathrm{H}(19 \mathrm{~A})$ & 2247 & 4590 & 343 & 46 \\
$\mathrm{H}(19 B)$ & 3934 & 5033 & 772 & 46 \\
$\mathrm{H}(20 \mathrm{~A})$ & 1632 & 6574 & 115 & 56 \\
$\mathrm{H}(20 \mathrm{~B})$ & -87 & 5944 & 785 & 56 \\
$\mathrm{H}(21 \mathrm{~A})$ & 633 & 7249 & 1446 & 64 \\
$\mathrm{H}(21 \mathrm{~B})$ & 2622 & 2440 & 64 \\
$\mathrm{H}(22 \mathrm{~A})$ & & & 42
\end{tabular}




$\begin{array}{lrrrr}\mathrm{H}(24 \mathrm{~A}) & 4486 & 2973 & 544 & 57 \\ \mathrm{H}(25 \mathrm{~A}) & 4968 & 1803 & -412 & 77 \\ \mathrm{H}(26 \mathrm{~A}) & 2705 & 513 & -383 & 87 \\ \mathrm{H}(27 \mathrm{~A}) & -42 & 372 & 642 & 81 \\ \mathrm{H}(28 \mathrm{~A}) & -535 & 1524 & 1610 & 58\end{array}$

